( 2,2

STUDY OF ENERGETIC ELECTRONS IN THE OUTER RADIATION-BELT REGIONS USING DATA OBTAINED BY THE LLL SPECTROMETER ON OGO-5 IN 1968

H. I. West, Jr.

R. M. Buck

(i. Davidson

MASTER

July 26, 1979

Work performed under the auspices of the U.S. Department of

Energy by the UCLLL under contract number W-7405-ENG-48.
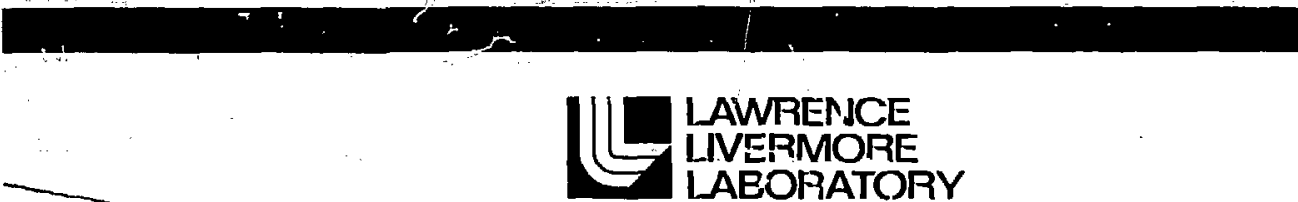

LAWRENJCE

LIVERMORE IABORATORY

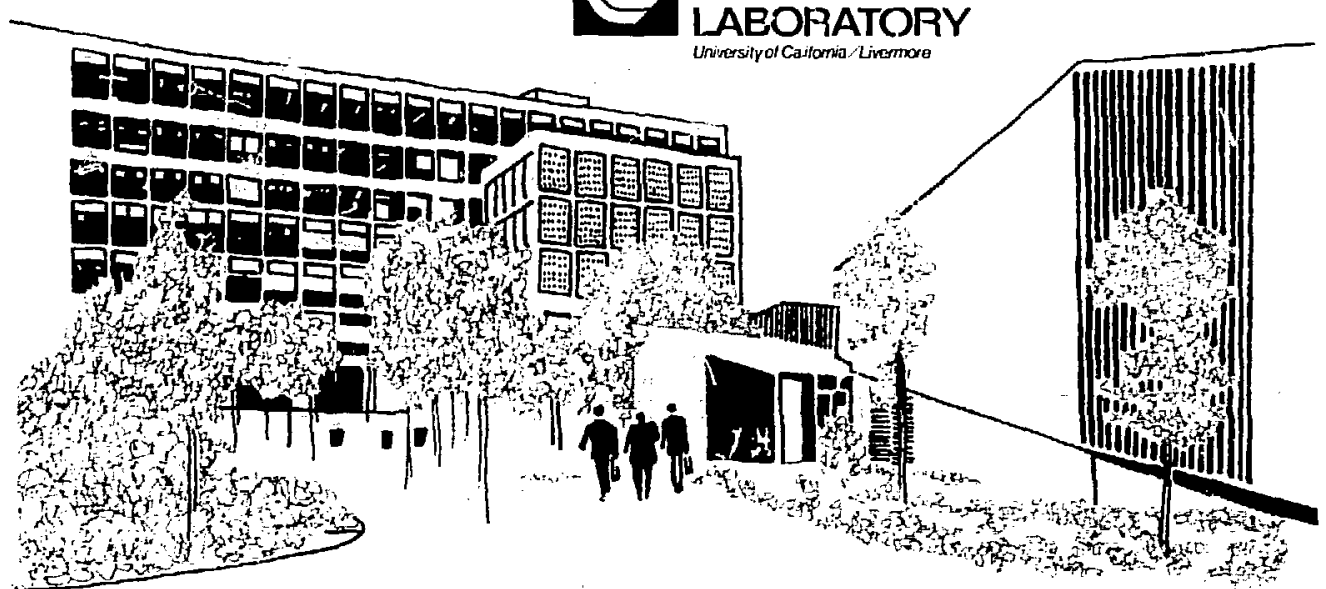




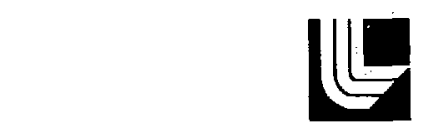

IAWRENCE LIVERMORE I_ABORATORY

University of Calfomia Livermore, California 94550

UCRL-52807

\section{STUDY OF ENERGETIC ELECTRONS IN THE OUTER RADIATION-BELT REGIONS USING DATA OBTAINED BY THE LLL SPECTROMETER ON OGO-5 IN 1968}

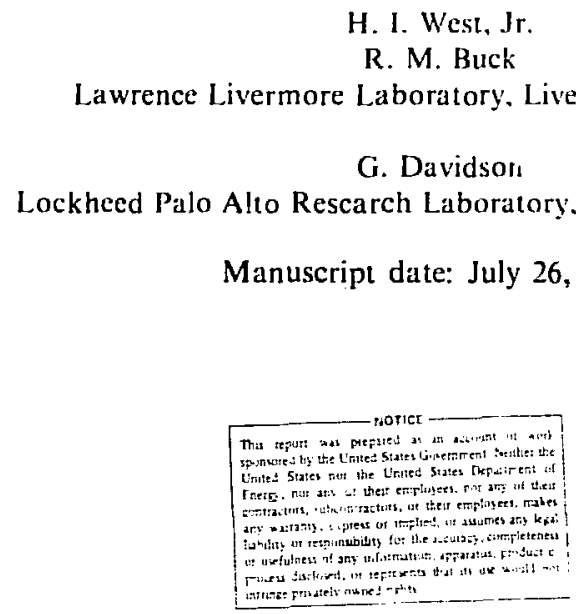


CONTENTS

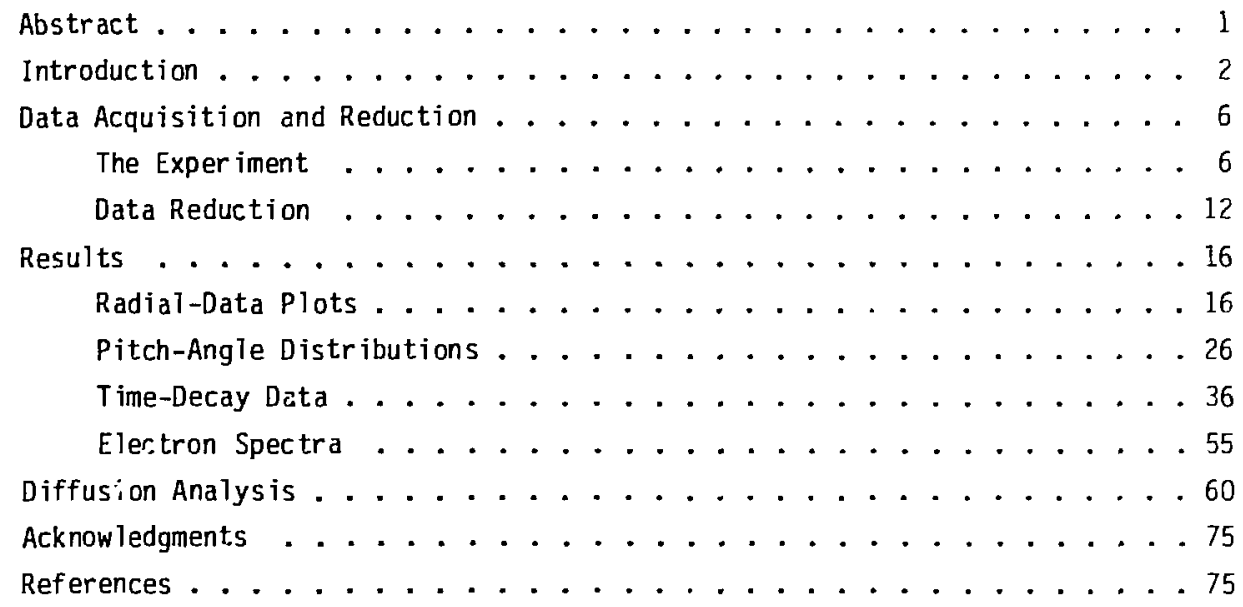


STUDY OF ENERGETIC ELECTRONS IN THE OUTER RADIATION-BELT REGIONS USING DATA OBTAINED BY THE LLL SPECTROMETER ON OG0-5 IN 1968

\section{ABSTRACT}

An account is given of measurements of electrons made by the LLL magnetic electron spectrometer ( 60 to $3000 \mathrm{keV}$ in seven differential energy channe15) on the 0go-5 satellite in the earth's outer-belt regions during 1968 and early 1969. The data were analyzed specifically to determine pitch-angle diffucion lifetimes as a function of energy in the L-range 2 to 5 . As a part of this effort, the general dynamics of these regions were studied in terms of the time-dependent energy spectra, and pitch-angle distributions for the seven energy groups were obtained as a function of $L$ with representative values presented for $L=2.5$ to 6 . The pitch-angle-diffusion results were used to analyze the dynamics of the electrons injected following the intense storms on October 31 and November 1, 1968, in terms of radial diffusion; the derived diffusion coefficients provide a quite reasonable picture of electron transport in the radiation belts. Both the radial- and pitch-angle-diffusion results are compared with earlier results. 


\section{INTRODUCTION}

Besides being of continued scientific interest, understanding the dynamics of the earth's radiation belts in terms of electron intensity, temporal behavior, and transport is very important with regard to radiation damage to satellites; the electrons can originate either from magnetic-storm injection or result from high-altitude nuclear detonations.

Measurements of the lifetimes of energetic electrons in the radiation belts are generally considered to be the key to understanding the transport of these particles. (See the last section of this report for the theory of particle transport.) There are two principal effects to be identified in the teinporal behavior of the electrons: those due to radial diffusion and those due to pitch-angle diffusion. This study was begun in an attempt to identify and determine the pitch-angle diffusion lifetimes at $L \geq 2$ to about $L=5$ at different electron energies (here, $L$ is the usual McIlwain shell parameter). In doing so, we have studied the dynamics of the outer radiation belt during two storm periods--one associated with weak injection on June 11, 1968, and one associated with major injection on October 31-November 1, 1968. This effort has required a complete study of the electrons present during these periods, including pitch-angle distributions (PAD's), temporal behavior of electron spectra, and, of course, the energy-dependent decay rates. We cover a) aspects of these data in this report and conclude with a diffusion analysis of energetic electrons observed during the November storm to show that we have indeed identified that part of the decay due to pitch-angle diffusion and that due to radial diffusion.

The measurements were made by the Lawrence Livermore Laboratory (LLI) experiment on the 0go-5 satellite, which was launched March 4, 1968, into an orbit of $23 R_{E}$ apogee and $-150 \mathrm{~km}$ perigee. Initialiy, apogee was at 1000 Local Time (LT), but during the year, the line of apsides precessed earlier in local time around the earth as a result of the earth's motion around the sun. Including the LLL experiment, Ogo 5 carried a complement of 22 scientific experiments.

The LLL experiment measured not only the energetic flux of electrons and protons but also their pitch-angle distributions (PAD's). To determine the PAD's, vector magnetometer data are required; fortunately, quality data covering almost all periods of data acquisition in 1968 and 1969 are available from the UCLA experiment [Aubry et al., 1972; P. J. Coleman, principal investigator]. These data were provided in a timely manner by $i . T$. Russell. 
Often in 1968, the inbound orbit of Ogo 5 was close to the magnetic equator from $L=10$ to 4 and sometimes into $L=3$ and, of course, the inner belt near perigee. This has afforded the unique opportunity to study the particle fluxes at these distances throughout the year. Although proton measurements were available from our experimeri, the proton detectors were of ten saturated at $L<5$ whereas the electron data were of consistently high quality. The electrons in the inner belt for 1968 [Buck and West, 1974; West and Buck, 1976ab] have already been studied. In addition, comprehensive studies of the electron PAD's have been reported for the equatorial reginns of the inagnetosphere [West et al., 1973bc; West and Buck, 1974]; the LLL electron sper tromoter results have also been reviewed [West, 1978].

We emphasize that the data coverage by the LLL spectrometer was unique. Although it is true that other experiments measuring electrons have been conducted in the regions of interest, e.g., the Minnesota magnetic spectrometer experiment on Ogo's 1 and 3 [Pfitzer and Winckler, 1962; 1966; Pfitzer et a1., 1966; Winckler, 1970], most of the reported experimental resilts are for the inner belt. Al1 too often, the measurements reported by various experimenters are from integral spectrometers, and this has limited the interpretation of the resulting data. A notable exception are the magnetic-spectrometer results obtained by Vampola (1972, private conmunication) using Air Force satellites. We use his data in the present study; the data were acquired far away from the magnetic equator, but this does not unduly complicate matters for the present purposes. Spectrometer data of various kinds have been collected at the National space Science Data Center and used for various engineering studies [Teague et al., 1976; Singley and Vette, 1972; Vette, 1972; Hilberg, 1974: Teague and Vet.te, 1974; Chan et al., 1979]. The engineering approach in their analysis, although expedient, has resulted in radiation-flux prediction codes that are, on the average, good to about an order of magnitude, but in no way allow assessment of fluxes following major injections, e.g., as from a "great" magnetic storn. Although the results presented here are intended to serve a utilitarian purpose, they are the result from careful work, which makes them of value to further scientific analysis.

In this study, we emphasize the periods during 1968 following the magnetic storms of June 11 (Day 163) and October 31 and November 1 (Days 305 and 306). The magnetic activity values (Dst) during the June storm were $-94 \gamma(3-h)$ and -61 Y (24-h average) compared with Dst values for October 31 - November 1 of 
? ? 0 and $-231 y$ and -105 and $-131 y$ for $3-h$ and $24-h$, respectively. (The Dst data used in this report were prepared by Sugiura and Poras [1971]).

The June storm produced well-defined rapid injection into $L \simeq 3$; this is to be compared to injection into $L \simeq 2$ observed during the October-November storms. For the June storm, it was possible to follow the decay after the injection, and, we believe, to measure the pitch-angle diffusion lifetimes, at least for the lower energy electron channels. Our study of the 0ctoberNovember periad is interesting in that, although strong effects of radial diffusion appear in the data, these resilts corroborate the results obtained for pitch-angle diffusion during the June storm.

To provide perspective for our study, it is worthwhile to examine inner-belt data obtained from the Applied Physics Laboratory (APL) experiment on Satellite $196338 \mathrm{C}$ during 1963 through 1968 [Bostrom et al., 1970] and our LLL results [West and Buck, 1975a; for inner-belt electron spectra, see West and Buck, 1976b]. The APL results shown in Fig. 1 for the electron fluxes $\geq 280 \mathrm{keV}$ were acquired at low altitudes. For our present purposes, we draw attention to the injection at $L=2.2$ following the major magnetic storms of the period. The storm of major interest to us here (Day 163, 1968) shows little injection. However, we clearly see the effects of injection at Days 305 to 306. The results of the LLL study are shown in Fig. 2 and are the

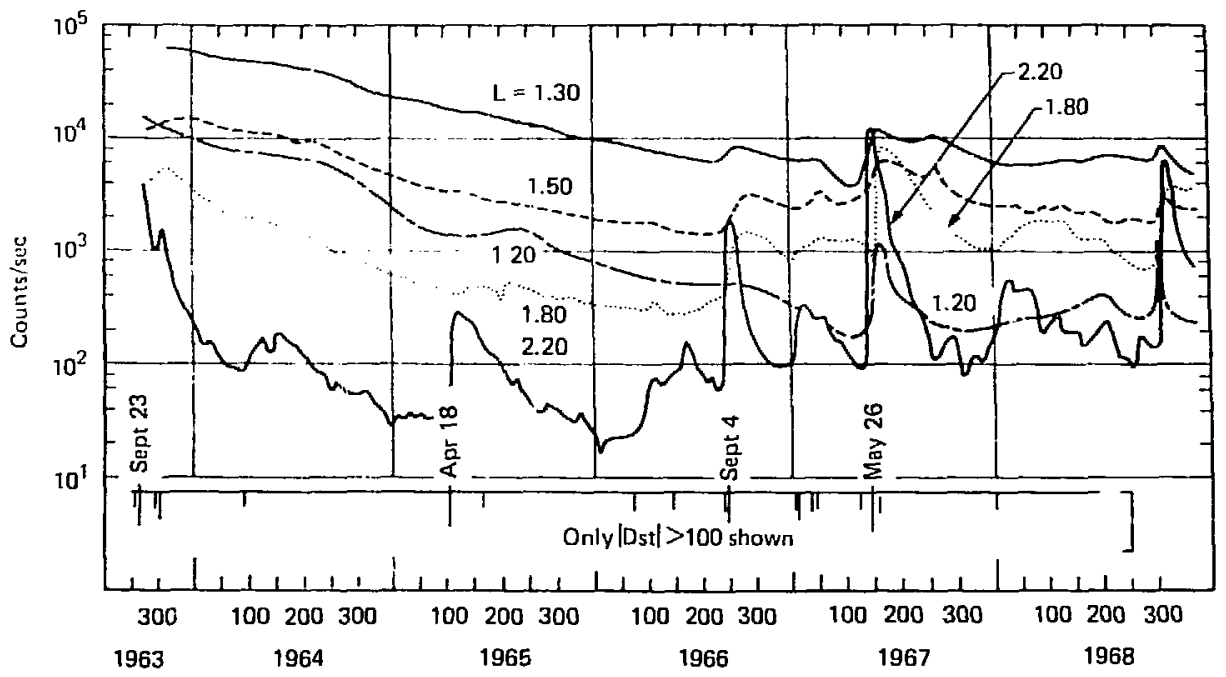

Fig. 1. Electron fluxes $>280 \mathrm{keV}$ obtained on satellite 1963-38C at low altitudes during 1963-1968 [Bostrom, 1970]. The data provide perspective for the present study. 


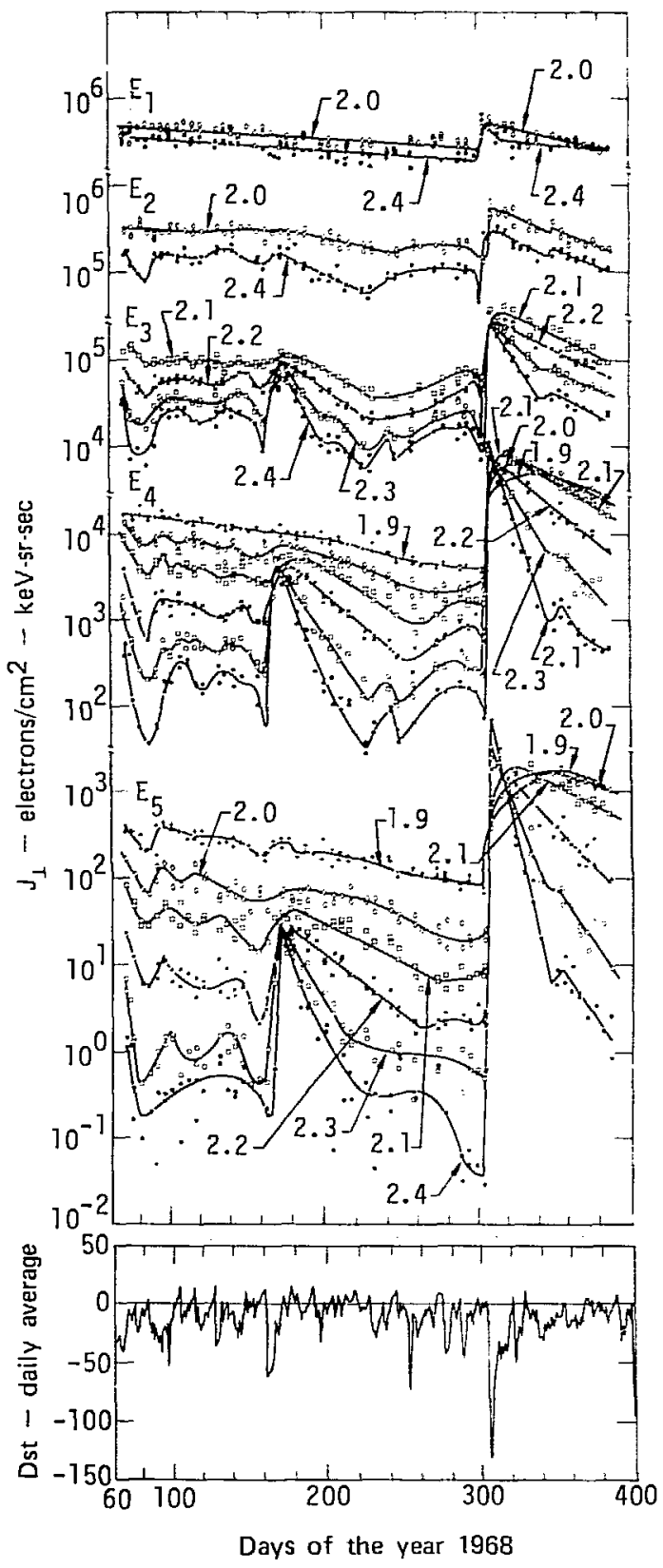

Fig. 2. Electron-decay data obtained by the LLL spectrometer at $L=1.9-2.4$ on 0go 5 during 1968 [West and Buck, 1976a]. The data are corrected to the equator. The periods of major interest follow injection on Day 163 and Days 305-306. 
result of an extensive analysis of time and spatial distributions. Figure 2 shows the equatorial $j_{\perp}$ fluxes vs time, and the well-defined injections for our two storms of interest are apparent. We believe that the rapid decays following the Days 305-306 injection are due predominantly to pitch-angle diffusion, as is brought out later.

\section{DATA ACQUISITION AND REDUCTION}

THE EXPERIMENT

A detailed description of the electron spectrometer has been presented by West et al., [1969, 1973a, 1973b], but, in the interest of completeness, we present some of the features here. Figures 3 and 4 show the two magnetic

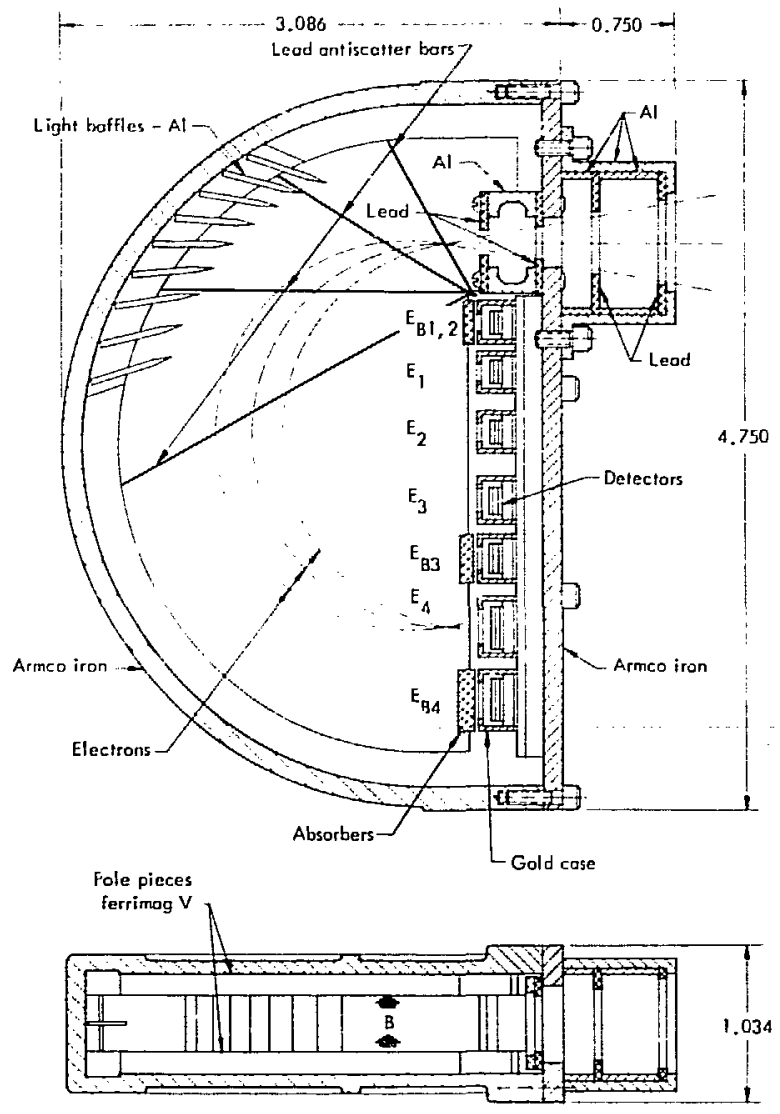

Fig. 3. Low-energy electron spectrometer. 


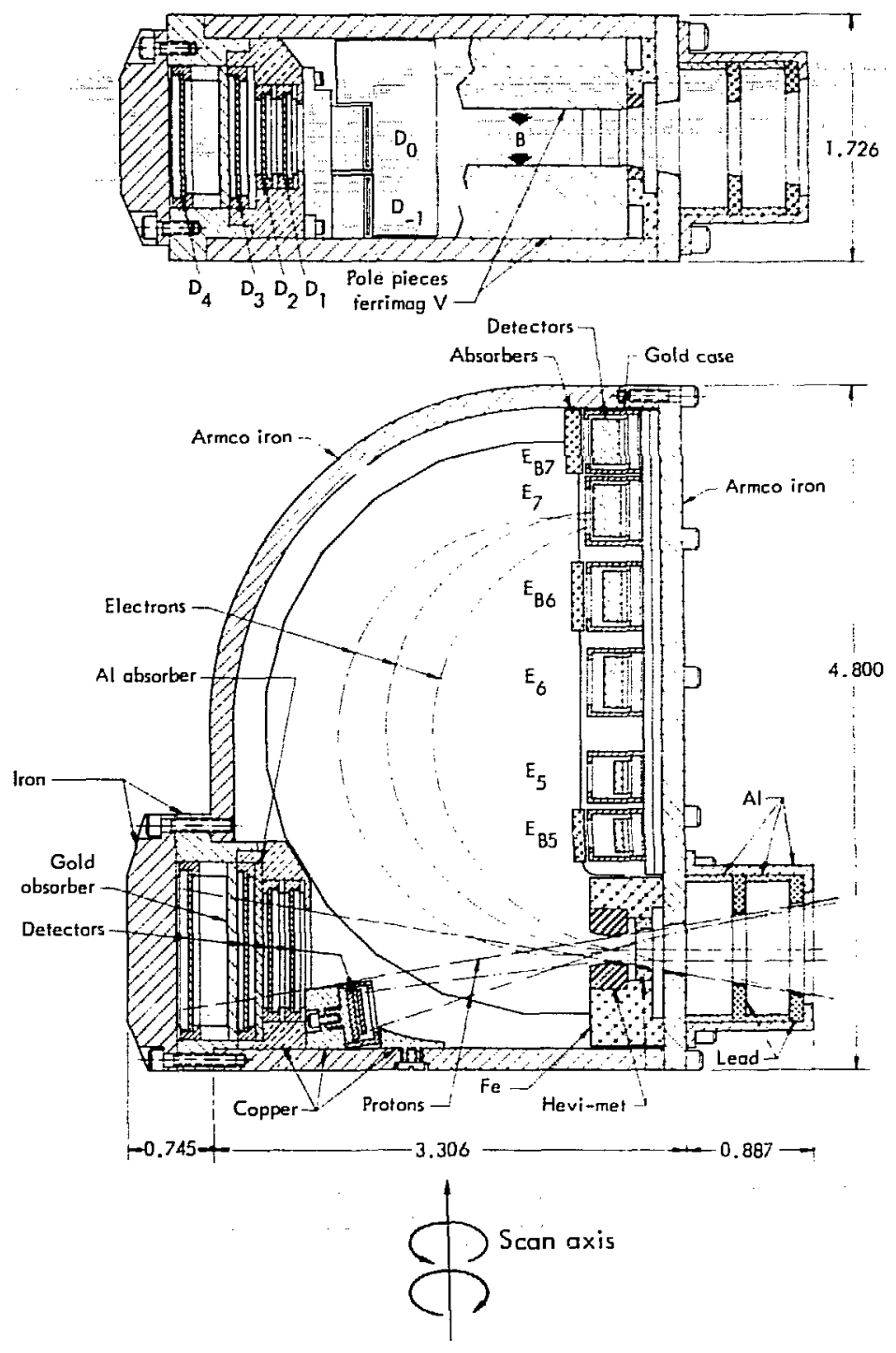

Fig. 4. High-energy electron spectrometer. The array of detectors in line with the entrance aperture is for proton detection; the results obtained using this spectrometer are not discussed in this report.

spectrometers containing solid-state detectors ior particle and background detection. Figure 3 shows electron channels $E_{1}-E_{4}$, and $F i g .4$ shows the high-energy channels $E_{5}-E_{7}$. We also note that the spectrometer in Fig. 4 
cuntains an array of solid-state detectors aligned with the entrance aperture ot the spectrometer. This array (telescope) was used for proton detection; data from this portion of the instrument were not used in the present investigation.

The spectrometer was designed to roject backgrounds from other radiations (penetratirig protons and bremsstrahlung) and to measure that background that could not be eliminated by good design; this aspect of the design is a very important froperty not shared by very many other instruments. The sensitive volune of the background detectors, pulse-handling electronics, etc., were identical to the corresponding electron detectors, and this has allo"ed rather accurate evaluation of the backgrounds. For example, the effectiveness of this reatment is demunstrated in the radial $j_{1}$-vs-L flux profiles (Figs. 8 and 9 :; the spectrometer channels of ten show zero count rates at $L \simeq 3$ in the presence of high count rates in other channels. In this respect, note that extremely intense proton fluxes were encountered at $L=3$.

$F$ :gure 5 shows the essential details of the space-craft coordinate sysiem. Dgo 5 was sun-earth oriented with the faces $\left(y_{p}\right)$ of the soiar paddles looking at the sun and the $+z_{b}$ axis of the space-craft hody pointec toward the center of the earth. The $L L L$ spectrometers were located on the hoOmi OPEP2 with the focal planes of the spectrometers lying in the $z_{e}$ direction. The OPEP2 scanned about the $Z_{e}$ axis so that the narrow fields of view of the spectrometers were scanned. An example of the scan-modulated data is shown in Fig. 6; the instantaneous pitch-angle of the spectrometer is read from the zig-zag pattern using the scale at the right. It is important to note that the fields of view of the spectrometers lay in the plane perpendicular to the radius vector $R$ extending from the earth through the space craft. This orientation limited the pitch-angle coverage in a dipole field to a range of $90^{\circ}$ to the local dip angle, $\tan ^{-1}\left(2 \tan \lambda_{m}\right)$, where $\lambda_{m}$ is the magnetic latitude. Hence, at high latitudes, i.e., $\lambda_{\mathrm{m}}>30^{\circ}$, the pitch-angle coverage was largely near local pitch angles of $90^{\circ}$. As a result of these Timitations, good PAD's were acquired only when Ogo 5 was near the equator, usually at invariant latitudes, $\lambda_{I}$, less than $10^{\circ}$ (an equatorial pitchangle, $\alpha_{0}$, of $-69^{\circ}$ ); the nomograph in Fig. 7 shows the relation between $\lambda_{I}, \alpha_{0}$ and $B / B_{0}$, where $B / B_{0}$ is the local-to-equatorial field ratio. of course, the constraints on the pitch-angle coverage were beyond our control, and we had to take the scan aveilable on the spacecraft. However, the scan that was provided did have its compensations for studies in the earth's magnetotal, and 


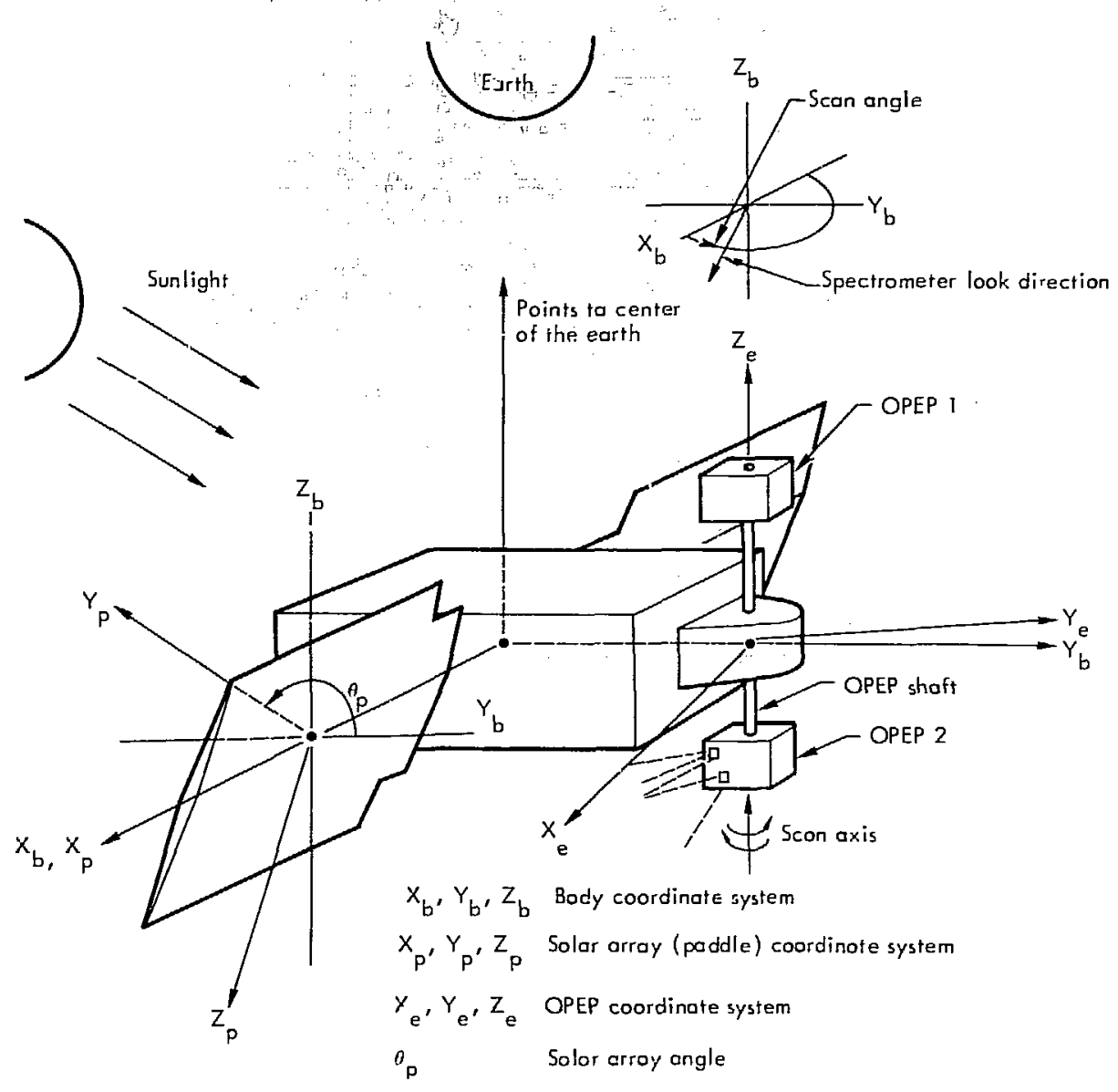

Fig. 5. Coordinate system showing ideal orientation of the $0 G 0$ spacecraft. Note that the spacecraft must be oriented so that the solar arrays look at the sun $\left(\phi_{p}\right.$ constrained to 90 to $\left.180^{\circ}\right)$ and $+Z_{b}$ points to the center of the earth. The experiment apertures on OPEP2 scan the $X_{b}, Y_{b} p l a n e$.

also, when 0go 5 was at righ latitudes near noon, near-circular field lines were encountered and especially good PAD coverage ranging from 0 to $180^{\circ}$ was afforded. The geometricul factors and the acceptance angles in the OPEP scan plane are given in Table 1. One would expect, and correctly so, that at positions away from the magnetic equator, the scanned acceptance angle increases, i.e., the effect of the wide fields of view in the focal planes of the spectrometers becomas an important part of the angular acceptance. However, this presents no pisblem since computational experiments that 


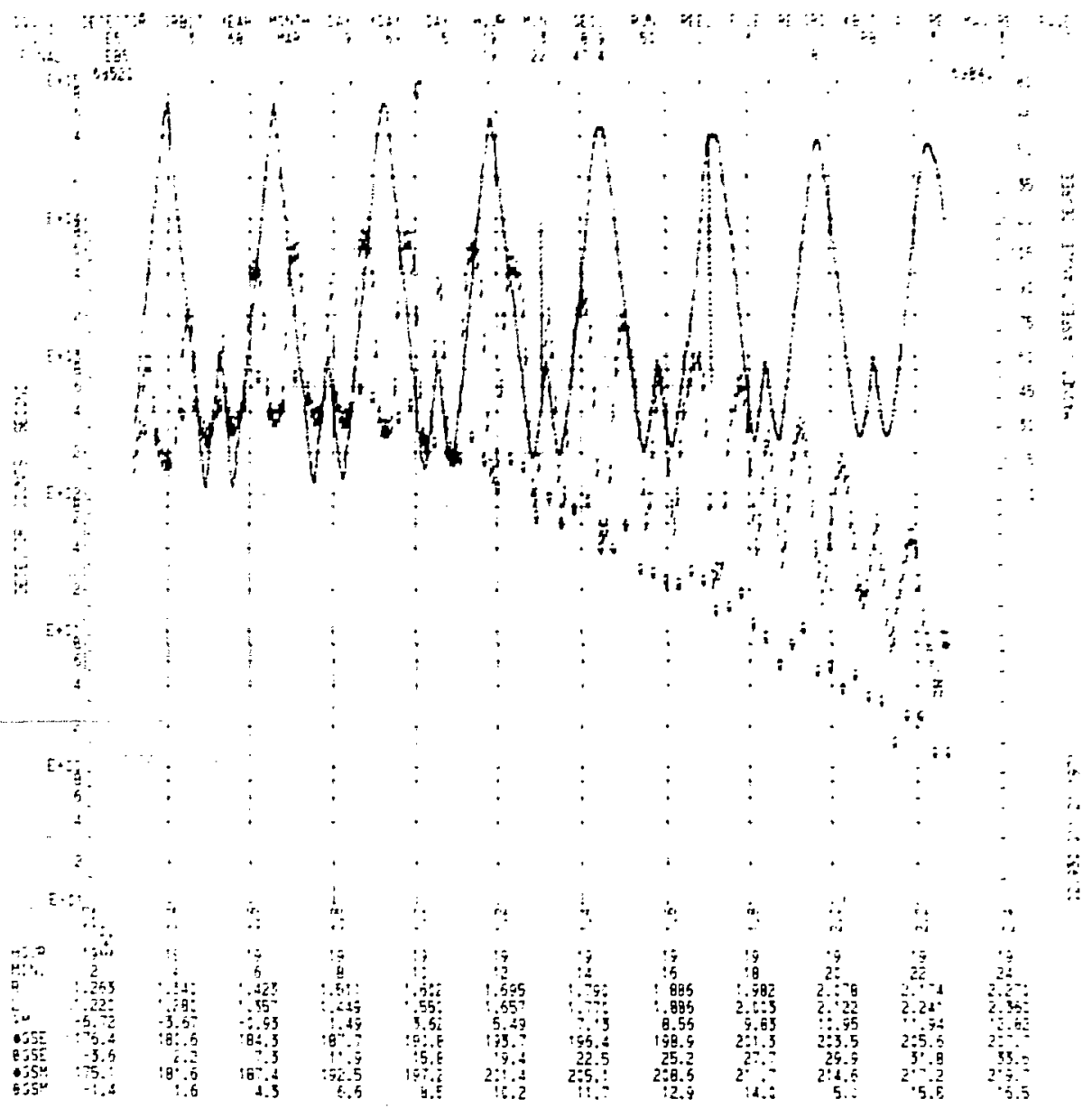

Fig. 6. Example of the scan-modulated electron fluxes. The l's represent the electron fluxes including background and the 0 's represent the background alone. The zig-zag patterı. gives the instantaneous look angle, $\ell$, of the spectrometer in relation to $\vec{B}$, to be read from the scale to the right.

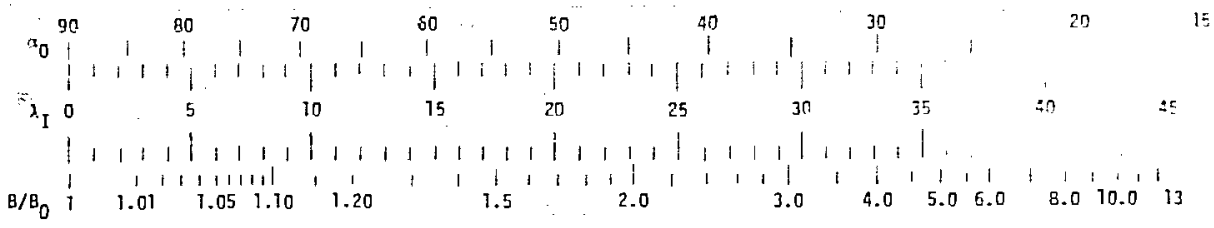

Fig. 7. Nomograph for $\alpha_{0}, \lambda_{I}$, and $B / B_{0}$. Here $\alpha_{0}$ is the equatorial pitch angle, $\lambda_{I}$ the invariant mirror latitude, and $B / B_{0}$ the ratio of local $B$ to equatorial $B_{0}$. 


\begin{tabular}{|c|c|c|c|}
\hline $\begin{array}{l}\text { Exortron } \\
\text { mannas }\end{array}$ & nnergy, bev & $\begin{array}{l}\text { Gronetry, } \\
\text { rnt }^{5} \text { bot si }\end{array}$ & $\begin{array}{l}\text { Acceptance } \\
\text { anglea, deg }\end{array}$ \\
\hline 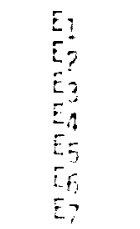 & $\begin{array}{r}79+23 \\
350 \pm 36 \\
260 \mp 36 \\
470 \mp 52 \\
822 \pm 135 \\
1530 \mp 260 \\
2830 \mp 270\end{array}$ & $\begin{array}{l}0.130 \\
0.277 \\
0.392 \\
0.605 \\
4.43 \\
3.57 \\
3.39\end{array}$ & $\begin{array}{l}7.6 \\
5.9 \\
4.7 \\
3.5 \\
5.3 \\
4.1 \\
2.5\end{array}$ \\
\hline
\end{tabular}

JEffective full width at 50\% acceptance in the scan plane.

simulate measured PAD's and the field of view of the spectrometer show the effect to be negligibie. Finite-resolution pho corrections are needed only when Dgo 5 was near the equator and we are assessing the PAD in the region near the lass cone $\left(0-20^{\circ}, z_{0}\right)$.

Two scan mechanisms were used on the OPEP. As a normal spacecraft function, the OPEP (both 1 and 2) was scanned at $1.2^{\circ} / 5$ with angular readout via selsyn resclvers. The spacecraft designers did not fully appreciate our need to know $\hat{\imath}$, the look Jirection of our spectrometer relative to $\vec{B}$ (节 was measured by the UCLA experiment on another boom), and hence the angular error in this readout could be $\pm 5^{\circ}$. Usually, however, this mechanism made only occasional jogs in its orientation in line with the operational needs of the spacecraft systems. Our laxperiment was located on a secondary scan mechanism (provided by the Goddard space Flight Center (GSFC)) that was rather accurately calibrated, although unfortunately in a relative manner because of the uncertainties in the orientation of the OPEP mechanism. However, if the GSFC mechanism was the only one operating (scanned at $3^{\circ} / \mathrm{s}$ through $230^{\circ}$ ), we can do a quite effective calibration of our spectrometers' $\hat{\imath}$ with respect to $\vec{B}$. We achieve this by letting the flux $j_{\perp}$ equal the symmetry point in the PAD's. This procedure provides a measure of the scan-plane correction. This procedure provides something more, however. Despite the efforts of the UCLA experimenters (C. T. Russell in particular), it was perfectly possible that small errors exist in the magnetic-field data and, furthermore, that an error of a degree or more may exist in relating these measurements to spacecraft body coordinates. Such errors are indeterminant and are combined in making the scan-angle correction (see below). 
On every other orbit inside of $-4 R_{E}$, the GSFC scan mechanism was turned off to accommodate a companion experiment, and our experiment's $\hat{l}$ was set to $-0^{\circ}$ in OPEP coordinates. The OPEP scan mechanism then kept $\hat{\imath}$ looking forward approximately in the orbital plane. In the following discussion, we describe the data acquired in this mode as non-scan data. Errors of $\pm 5^{\mathrm{c}}$ in the determination of the local pitch-angle may exist but are not consiuered serious in the following work.

\section{DATA REDUCTION}

Data are available in what we call the three-way mersiu tapes. Data w're available from the spacecraft at 1-kilobit (kb), 3-kb and 64-kb acquisition rates. Because of the commutation of our data channels, the data at the 1-kb rate represent counting periods of $4.608 \mathrm{~s}$ with readout every $4.608 \mathrm{~s}$. To compress the data, the data collected at the higher acquisition rates were averaged at $2.304 \mathrm{~s}$ (or 2-kb equivalent); this rate was chosen to maintain reasonable pitch-angle resolution. The three-way merged tapes [ Zeligman and Walton, 1972] then consist of files of the described counting-rate data interspersed with spacecraft attitude orbit $(A / D)$ and ragnetometer data (4.608-s averages).

The primary data-processing code, called BJPRP, obtains $j_{\perp} v \Sigma \lambda_{I}$ and extracts all available pitch-angle data. This code does the following:

(1) Recognizes when the experiment is scanning; (if it is not, it goes to $(6))$.

(2) Checks to see that the pitch-angle coverage is adequate for PAD analysis.

(3) Determines the simmetry point in the PAD of $E_{1}$ and/or. $E_{2}$ data (the symmetry point would be $90^{\circ}$ if not for the errors described earlier).

(4) Performs least-squares analysis of the available (after backgrounds are subtracted) PAD data, or,

(5) if the pitch-angle coverage is small but near $90^{\circ}$, selects the average flux near $90^{\circ}$ to $B$ for $j_{\perp}$, generated for $E_{1}$ through $E_{7}$ as a single data record.

(6) Determines the average flux in the non-scan mode every $77 \mathrm{~s}$ and subtracts the backgrounds.

(7) Calculates the invariant mirror latitude of each non-scan data point, which becomes part of the output along with the $j_{\perp}$ values. 
We describe the BJPRP operations on the sca'l data in more detail:

(1) The code first isolates a complete Pad from the continuous stream of $j$ (particle flux)-vs- $\alpha$ (apparent pitch-angle) data on the three-way merged tape. A complete PAD exists in the range 0 to $180^{\circ}$ and must include the $\alpha=90^{\circ}$ point; it allows no pitch-angle reversals (PAD is single-valued).

(2) Using the non-linear least-squares routine CURFIT, the PAD's for detectors $E_{1}$ and $E_{2}$ are each fit to a functional form

$$
\log _{10} j=a_{2}+a_{3}\left(\alpha-a_{1}\right)^{2}+a_{4}\left(\alpha-a_{1}\right)^{4}
$$

to determine the unknowns $a_{1}, a_{2}, a_{3}, a_{4}$; the $a_{4}$ term is omitted for 1-kb data. (These equations do not correct for the finite angular response of the spectrometer in any way.)

(3) The values of $a_{1}$ from the two fits are used to estimate $\alpha_{1}$, the apparent $P_{R}$. symetry point. The apparent pitch-angle values are then appropriately cormected to transform $a_{1}$ to $90^{\circ}$, and true pitch-angle values are ohtaines over the entire PAD.

(4) Using the linear least-squares routine MLR, a backwards regression analysis is performed on each detector $E_{1}-E_{7}$. Each PAD is first fit to a function of the form:

$$
\log _{10} j=b_{1}+b_{2}(\alpha-30)+\sum_{i=3}^{7} b_{i}(\alpha-90)^{2(i-2)} .
$$

Each coefficient is t-test: d for significance in the following way. Note that $\left|\left(b_{j}\right) / \sigma\left(b_{j}\right)\right|$ follows a t-distribution if $\left\langle b_{j}\right\rangle=0$. Hence, a value of $\left|\left(b_{j}\right) / \sigma\left(b_{j}\right)\right|>B$ implies that $\left\langle b_{j}\right\rangle \neq 0$ with a probability obtained from the $t$-distribution that depends on $B$ (a code input) and the number of degrees of freedom (dof) in the fit equal to the number of PAD points minus the number of terms in the fitting function. For $B=2$ ( $s^{2}$ andard input), a value of $\left|\left(b_{i}\right) / \sigma\left(b_{j}\right)\right|>2$ implies that $\left\langle b_{i}\right\rangle \neq 0$ with a probability of $95 \%$ (if dof $=2$ ) or $90 \%$ (if dof $=5$ ).

All coefficients are tested in this way to determine if they differ significantly from zero. If some fail the test, the term corresponding to the least-significant coefficient is dropped from the fitting function, and the fit is recalculated. This procedure continues until all coefficients are significant or only one remains.

The even-power terms insure a PAD form that is symmetrical about $\alpha=90^{\circ}$. The linear term ("detrending" term) is introduced to compensate for asymmetry" 
dus to the motion of the satellite through spatial flux gradients during the finite observatiun time of the PAD data.

(5) The finel fit of each PAD is evaluated, and the fit variance calculated every $5^{\circ}$ irom $a=90^{\circ}$ to the extremum pitch-angle position. The detrend term is deleted in calculating the count rates going to the data base.

(6) The calculated results and relevant ephemeris and magnetometer deta are written into a data-base disk file. The make-up of the data file is as follows:

\begin{tabular}{|c|c|c|c|c|c|c|}
\hline Word 1 & $j_{!}^{12}\left(E_{1}\right)$ & $\mathrm{j}_{1}\left(\mathrm{E}_{2}\right)$ & $j_{1}^{12}\left(E_{3}\right)$ & $j_{1}\left(E_{4}\right)$ & $\begin{array}{l}12 \text { bits } \\
j_{1}\left(E_{5}\right)\end{array}$ & \\
\hline Word 2 & $\mathrm{j}_{1}\left(E_{6}\right)$ & $\mathrm{j}_{1}\left(E_{7}\right)$ & $\begin{array}{l}12 \\
\lambda_{1}\end{array}$ & 11 & $\begin{array}{c}4 \\
0 \text { or } 1 \\
\text { ishleg }\end{array}$ & $\begin{array}{c}3 \\
\text { scan } \\
\text { mode }\end{array}$ \\
\hline & 21 & 18 & 19 & 2 & & \\
\hline
\end{tabular}

Word 3

L R

$|\bar{B}|$

kb rate

0 or 1

Word 4

$$
\text { ms of day UT }
$$

9

day

7
year

17

18

18

18

6

Word 5

中GSM

longitude

$\lambda_{\mathrm{GSM}}$

$\lambda_{\mathrm{m}}$

confidence latitude magnetic latitude

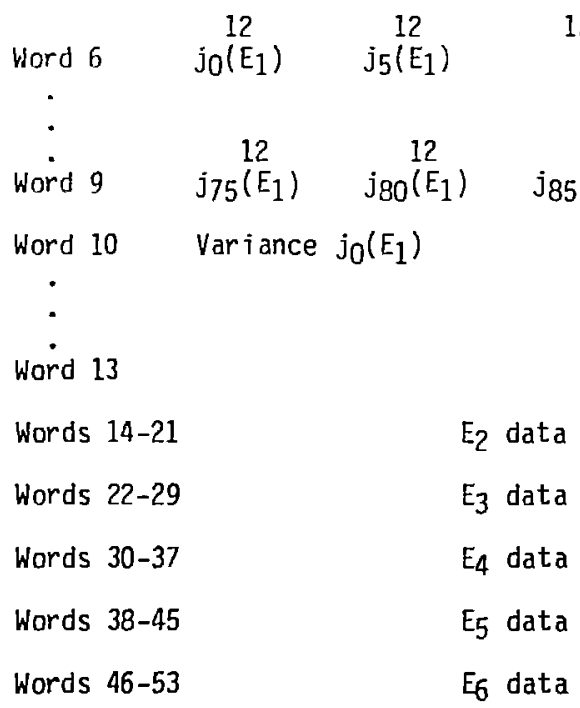


sinds $5 h-61$

E J Jata

hord 62

$\begin{array}{rrrrr}5 & 6 & 6 & 6 & \\ E_{1} & E_{2} & E_{3} & E_{4} & E_{5}\end{array}$

Word 63

BX $\stackrel{20}{20}(U C L A)$

$\begin{array}{cr}6 & 6 \\ E_{5} & E_{6}\end{array}$

6
$E_{7}$

$\begin{array}{ccc}2 & 7 & 9 \\ \text { I } & \text { INO } & \text { I } \\ \text { Predict } & \text { Bkg } & \text { Paritysum }\end{array}$

Word $54 \quad$ Geographic latitude $\quad 20 \quad 20$

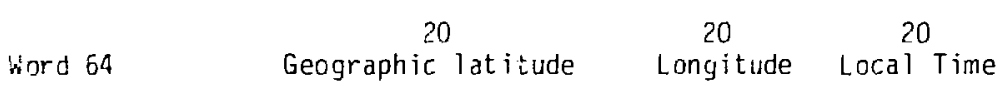

Word $54 \quad$ Geographic latitude $\quad 20 \quad 20$

20

20

BY BZ

The data in words 3 and $4,|\bar{B}|, B / B_{0}$, and $L$, are taken from the field model. In Word $2, a_{1}$ is the symmetry point of the PAD's before correction, and $\lambda_{1}$ is the invariant mirror latitude of the fluxes in question calculated from $B / B_{0}$ (i.e., $B / B_{0}=\left(1 / \cos ^{6} \lambda_{I}\right)\left(A-3 \cos ^{2} \lambda_{I}\right)^{\frac{1}{2}}$ for scan data). To obtain the mirror latitude for non-scan data, we replace $B / B_{0}$ by $\left(B / B_{0}\right)\left(1 / \sin ^{2} \alpha_{a v}\right)$ in the above expression; here $\alpha_{a v}$ is the average pitch-angle of observation found in the a location. In these calculations, we have relied upon the results of the field model provided on our $A / 0$ tapes. Inaccuracies clearly occur at $L>4$. The 0150 -Pfitzer model [015on and Pfitzer, 1974; private communication, 1972], an improved model, would be much better since it is veighted heavily by magnet umeter results obtained at $6.6 \mathrm{R}_{\mathrm{E}}$ and allows for day-night asymmetries. We rave not yet used this model in our studies.

Word 62 contains diagnostic data: $E_{1}-E_{7}$ give the number of data points per PAD; I Predict equals one if predict B-values are used because of flux-gate magnetoneter fill-words and equals zero otherwise; INO Bkg is a flag that is set if the final fit is zeroth order; and I Paritysums is a running count of the tape-parity errors.

We found that outputing the individually fitted PAD's for each channel on microfiche has made for rapid retrieval during the course of this work, and this should be valuable for subsequent work. We later show PAD's obtained throughout the magnetosphere as a short but incomplete study of such results.

The above file is outputed as a J-FILE and also becomes part of a database file called NEWDB. This file results in much greater data packing than the J-FILE and provides a directory and history of data processing. To facilitate manipulation of NEWDB, a code called NDBALT was constructed that allows data-transfer between files NEWDBX and a NEWDBY and that inserts or extracts J-FILES. 
Various plotting routines are controlled by the code BPLTM. The code ip-DATA produces plots of $j_{1}$ vs $L$ operating on the J-FILES. The codes J0BO9 and JOB 10 produces rumerical listings of the essential data in a J-FILE excluding pitch-angle data (these codes also work directly from NEWDB). Of special interest is $30 \mathrm{BO}$, which produces pitch-angle plots by operating on a 1]-FILE. The pitch-angle plots are $j(\alpha)$ vs $\alpha, j\left(\alpha_{0}\right)$ vs $\alpha_{0}, j\left(B / B_{0}\right)$ vs $B / B_{0}$, and data Lables. These plots are available on both microfiche and 35-mm microfilm.

The codes J0BO9 and JOB10 were designed for editing all data except those in the PAD portion of the file. In this case, the data of interest are the $j_{\perp}$ values and attendant data. Corrections are inserted by cards or, potentially, by teletype.

\section{RESULTS}

RADIAL-DATA PLOTS

Figures $8 a-d$ show examples of data for the Day-163 storm-injection period plotted by the TP-DATA option of the BPLTM code. The plots show $j_{1}$ vs $L$ for $E_{1}-E_{7}$ along with $\Phi_{G S M} v s L$ and $\lambda_{I} v s L$. We note that the $\lambda_{I}$ curves show points (the smooth curves) along the satellite orbits at the positions of available count-rate data; for the non-scan portions of a given orbit at $\mathrm{L}>4$, there are additional data points at greater latitude that indicate the mirror latitudes of the non-scan data. The count-rate plots are presented with l's marking the data points on the intsound orbits and 0 's on the outbound orbit. (Because the points are not reproduced well, the respective legs of the orbits are marked with arrows.) To allow the presentation of backgrounds, the data are presented as true count rates plus 1 . The data were initially processed with $+2 \mathrm{c} / \mathrm{s}$ to facilitate background subtractions and use of the logarithms of the count rates; these data are stored with the $+2 \mathrm{c} / \mathrm{s}$ in the data base.

We show the Day-158 data in Fig. $8 \mathrm{a}$ as an example of data obtained before the Day-163 storm injection. An important feature is the energy dependence in the slot position $(L=2-4)$ and its depth, i.e., relative intensity of the electrons in the slot. For $E_{4}-E_{7}$, the fluxes in the slot are zero within statistics. For $L>5$, there are marked differences in the particle intensities between the inbound and outbound portions of the orbit. For the outbound portion, the satellite was closer to midnight (note the value of $\phi_{G S M} ; 180^{\circ}$ is equivalent. to local midnight) and at higher latitude. 


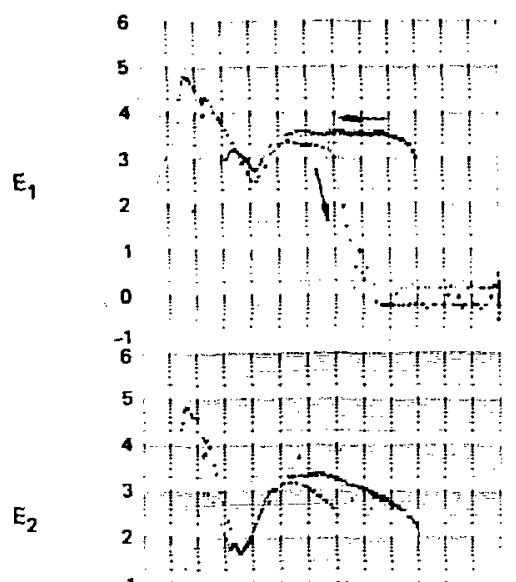

$1 \div 1 \cdot 1 \cdots+1,1+1 !$

o. 1

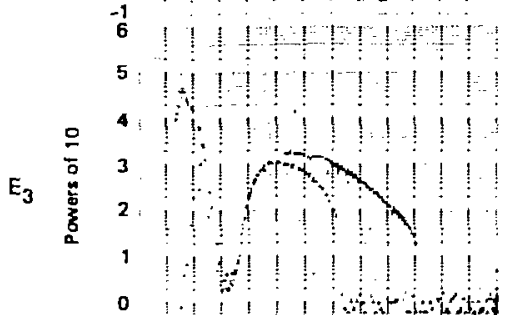

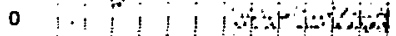
$-1$

5

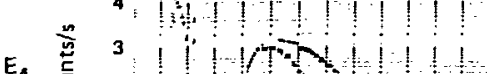

$E_{4}$

2111

$1,1 \cdot 1+1$

$01+1119+19+4$

6101

5. $1=1$

$441+1=1$

$32+1,13=1$

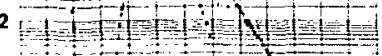

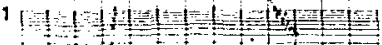

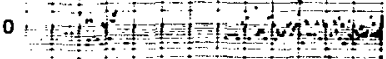

${ }^{-1} 0$

L

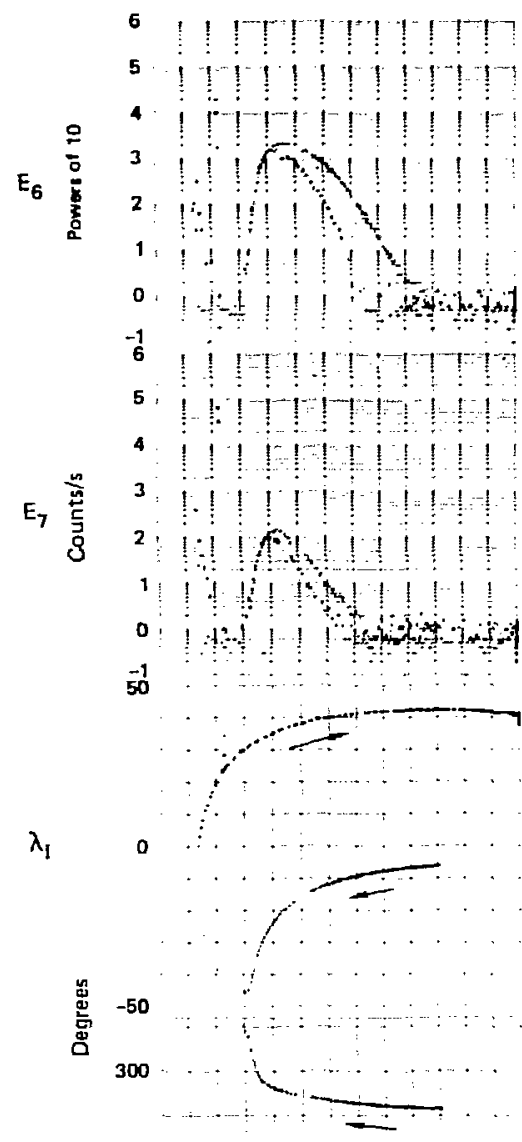

$\phi_{\mathrm{GSM}}$

200

100

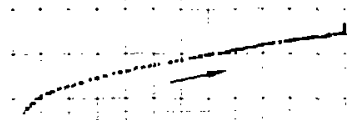

0

L

13
Fig. 8a. Radial $j_{1}-v s-L$ plots for Day 158,1968 . These are quitt ime data prestorm. The 1 's and 0 's indicate the inbound and outbound orbits, respectively; the legs of the orbit are further emphasizer by the arrows on the drawings. 

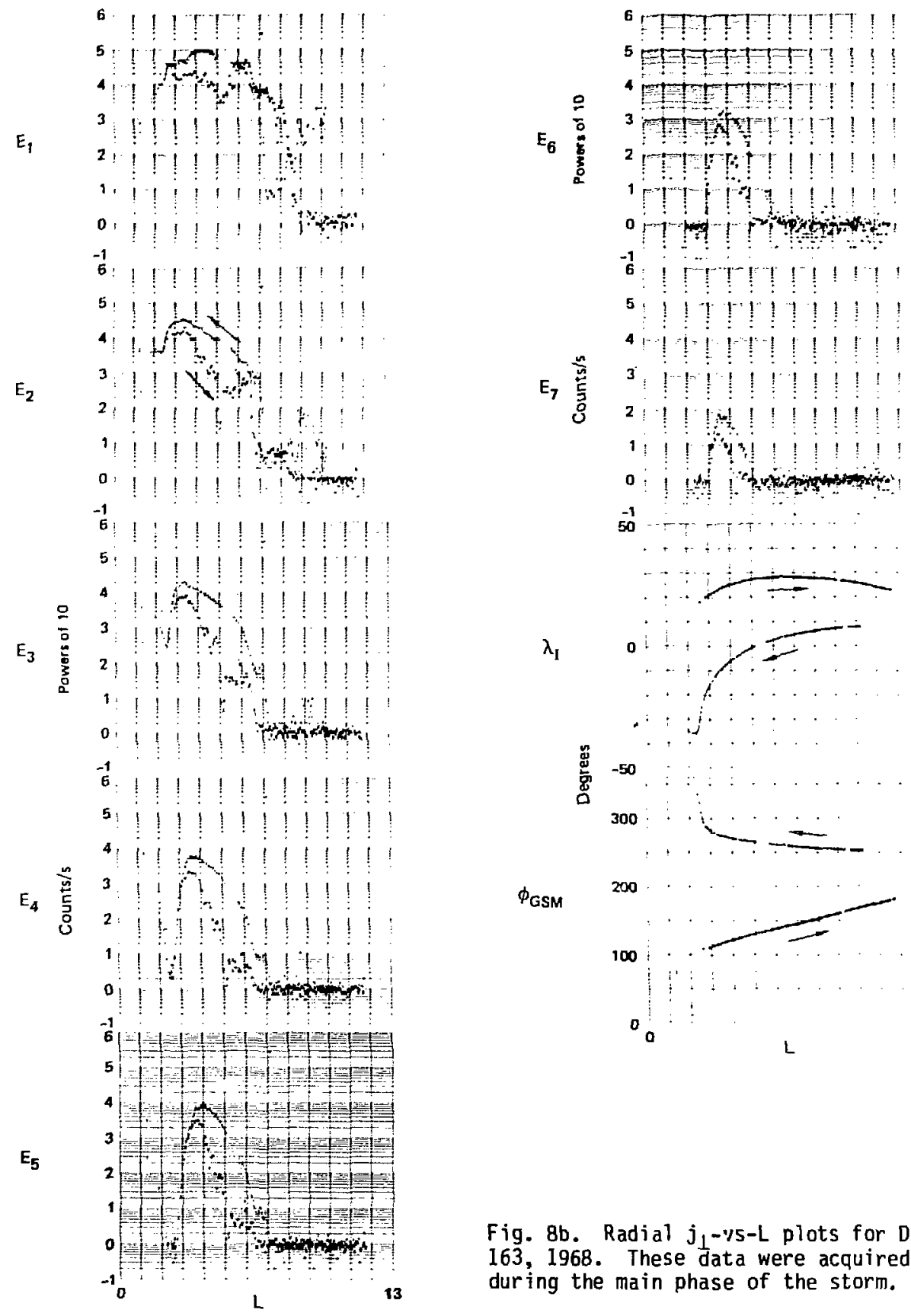

13

Fig. 8b. Radial $j_{1}-1 / 5-L$ plots for Day 163,1968 . These data were acquired during the main phase of the storm. 

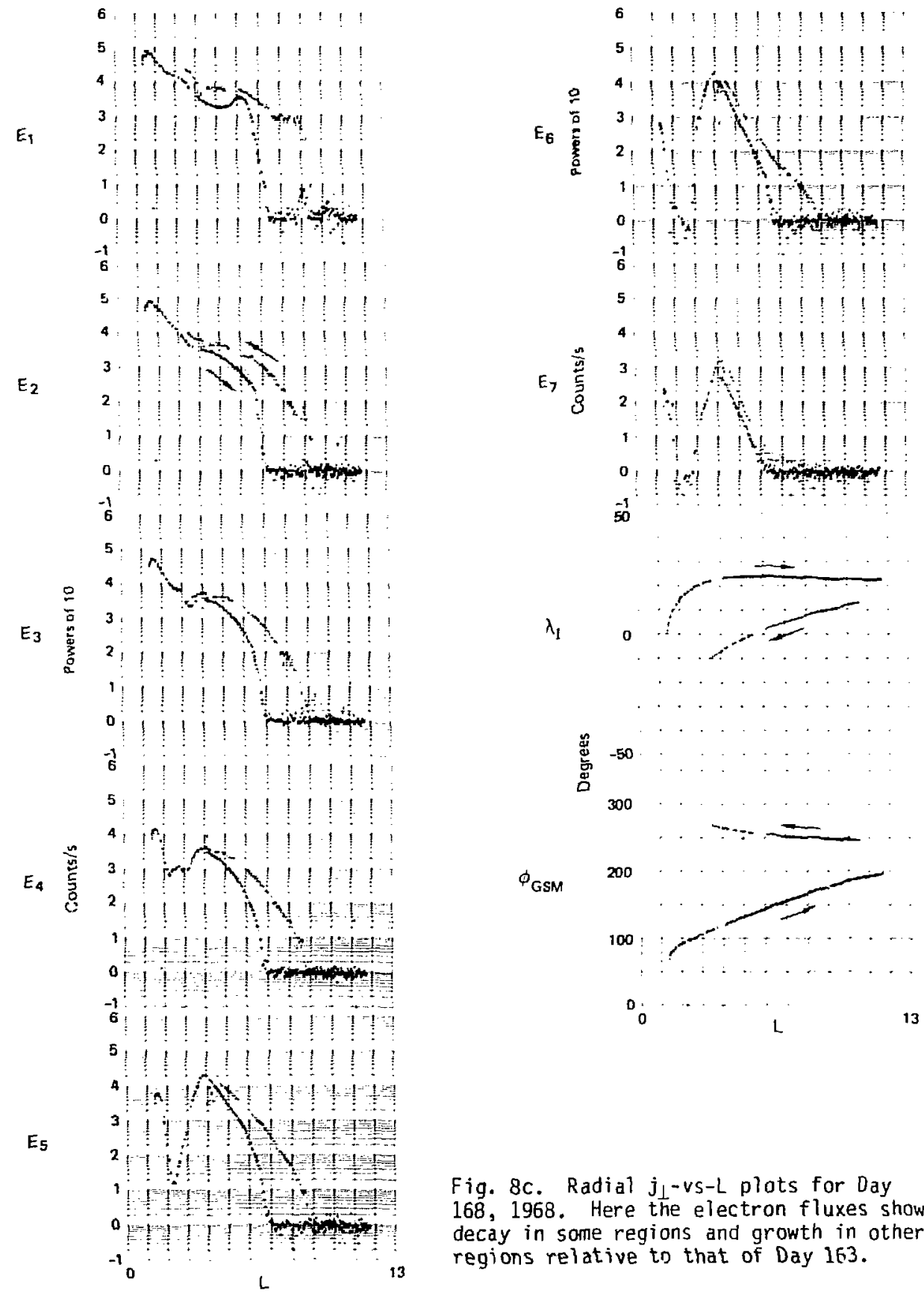

Fig. 8c. Radial $j_{\perp}-v s-L$ plots for Day 168,1968 . Here the electron fluxes show decay in some regions and growth in other regions relative to that of Day 163 . 


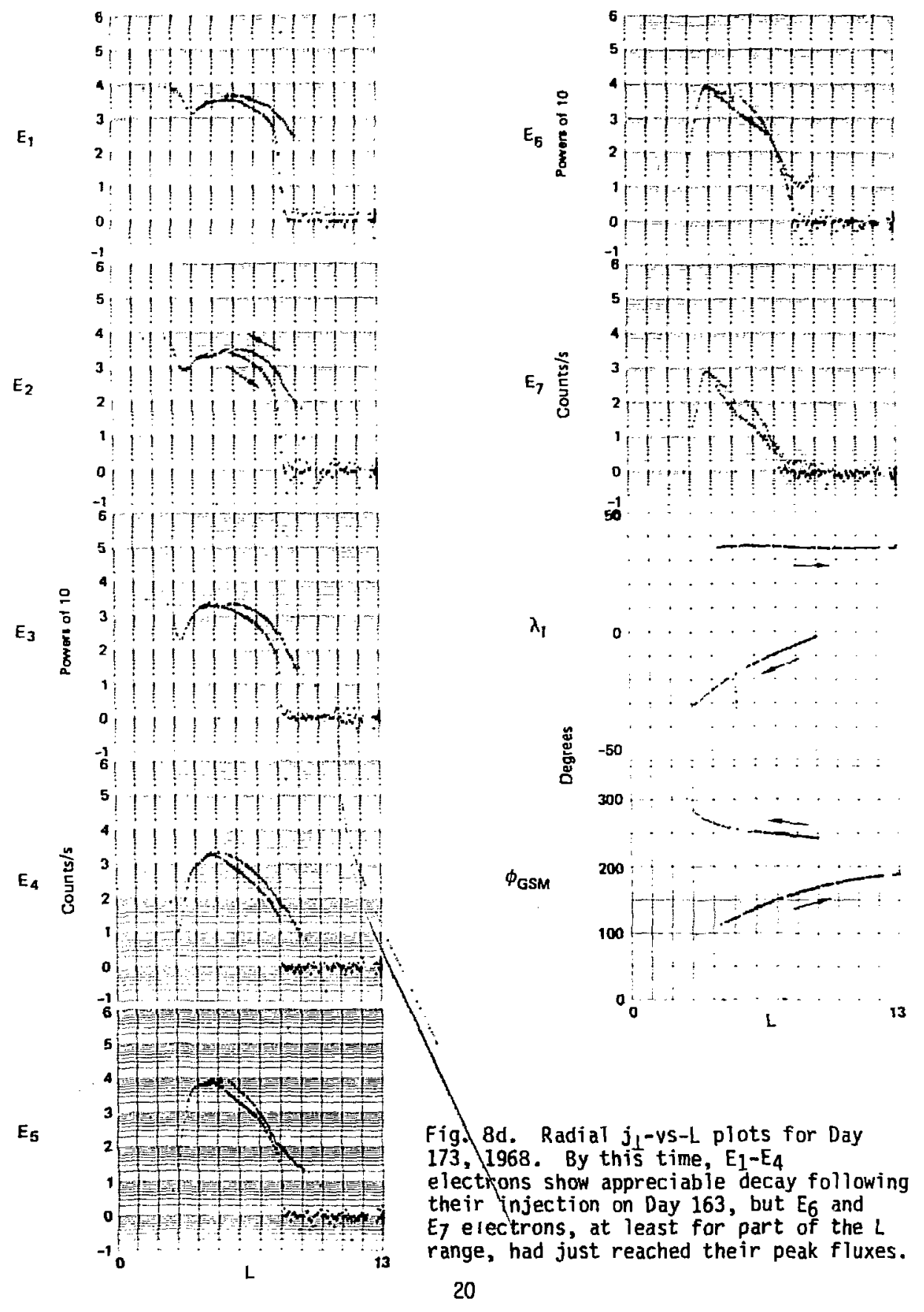


The data of Fig. 8a should be compared with those of Fig. 8b that were acquired during the main phase of the magnetic storm on Day 163 . The effects of the injection are particularly evident at $L=3$, especially in $E_{4}$, where the increase is from about 0 to $500 \mathrm{c} / \mathrm{s}$, and in $E_{3}$, with changes from 35 to about $10^{4} \mathrm{c} / \mathrm{s}$.

The regions of greatest counting-rate fluctuations in the Day 163-data are on the outbound leg of the orbit. Figure $8 \mathrm{~b}$ indicates that 0 go 5 was near midnight with $\lambda_{1}=20-30^{\circ}$. (The field distortions were so great in this case that $L$ was not a particularly good parameter for $L>3.5$.) Apparently we were witnessing the main-phase injection of energetic particles during this orbit.

Figures 8cd s'inw data for Days 168 and 173. The Day-168 óata are representative of conditions after the magnetosphere had settled down following injection and the particle fluxes had begun their rearrangement as a result of pitch-angle and radial diffusion. It is instructive to notice the evolution of the slot $(L=3-4)$ in the data of the :rious energy channels uetween the data of Days 168 and 173: for a given ent^gy, the slot begins at that $L$ shell in which, as a result of losses caused by pitch-angle diffusion, electrons have their minimum lifetime. (For $E_{1}-E_{3}$ results, the slot is latitude-dependent for these data in this respect.) It is probably significant that for the $E_{4}$ lata of Day 168 , two minima are present (at $L=2.3$ and 3.0 ). The dual minima also appear ir data for Day 171 (the next. available data), but the decay effects are too rapid for a good assessment of the decay times; the two data sets are combined in the decay studies.

Figures 9a-d show radial flux plots, or the period associated with the November-0ctober magnetic storms. Figure 9 a shows data fur Day 301 . Note in particular that for $E_{5}, E_{6}$ and $E_{7}$, there are no electrons at $L=3$. Figure $9 \mathrm{~b}$ shows data on Day 300 during the main phase of the November 1 storm. Here we note rather strcig injection into $L=2.5$ in all channels, but especially the higher energy channels, with the exception of $E_{7}$. For bot $n$ the June and October-November storms, $E_{7}$ electrons were lost during the main phase, later to re-emerge, ostensibly, as a result of radial diffusion or possibly some more rapid mechanism. Such loss is usually attributed to parasitic wave-particle interactions at the plasma pause [Thorne and Kenrel, 1971], but this explanation has been called into question by Davidson [1978]. In comparing particle fluxes between the two storms for Days 163 and 306 , it should be noted that the latter data were acquired farther from the magnetic 

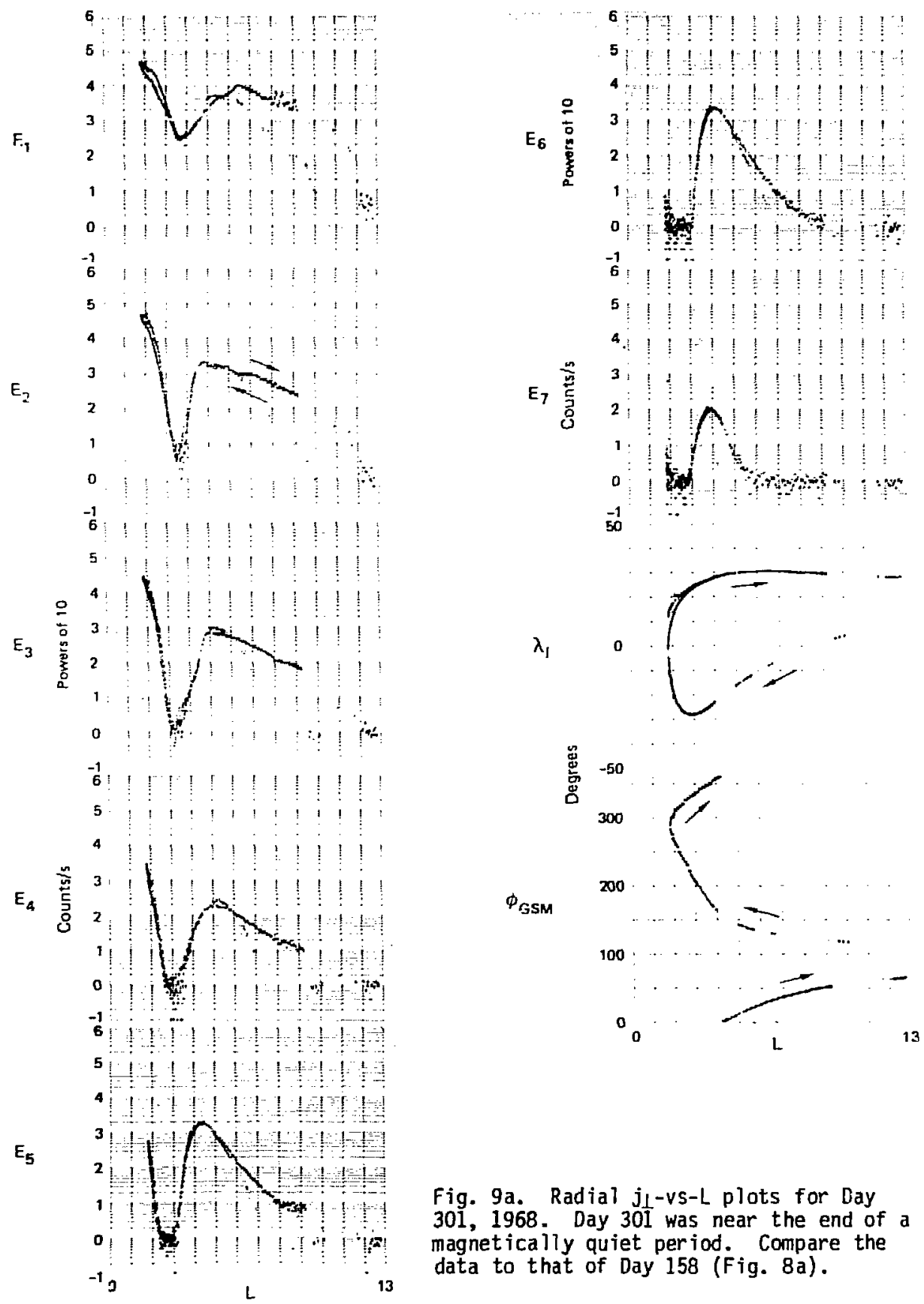

Fig. 9a. Radial $j_{\perp}-v s-L$ plots for Day 301, 1968. Day 301 was near the end of a magnetically quiet period. Compare the data to that of Day 158 (Fig. 8a). 


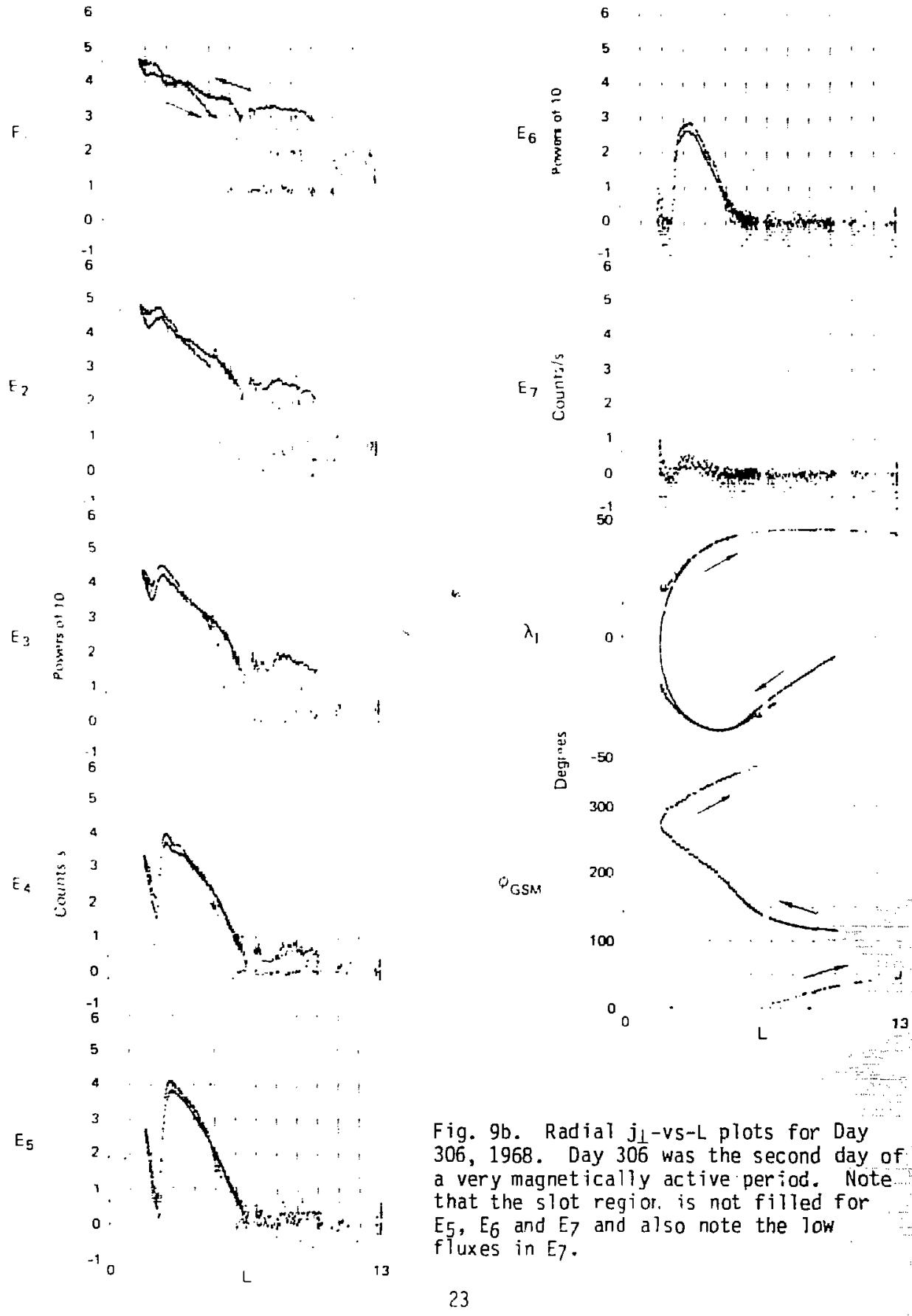


$E_{1}$

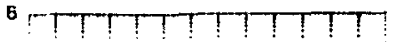

$5, \cdots+T \div+T \div \div$

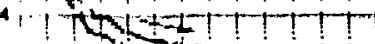

$311-7$ int?

$2,1-190$

1 1 $1 / 1,1+1+1+1$

$0: 1: 1101 \%$

$-1$

TITI!

${ }^{5} \mathrm{Tt}+\mathrm{CH}+1$

${ }_{4}^{4} 1+1010+1$

$E_{2} \quad 3 \quad,-9+4+1=$

$21-1-1+1$

$1111-10$

$0 \quad i \quad i[i]+i]$

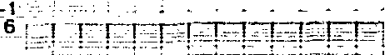

$1+1+1$

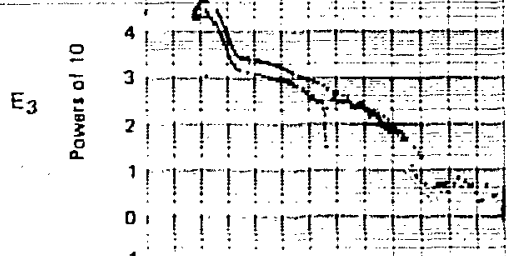

$-1$

TI ए।

$5 \mathrm{~m}+1$

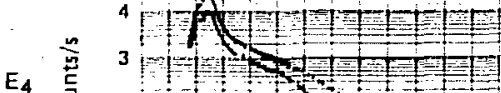

$210101+0$

$1-1+1-1+2]$

0101010

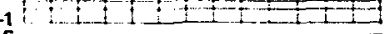

6 年1

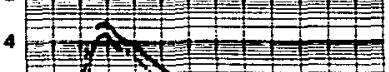

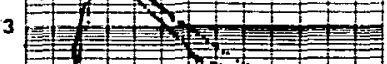

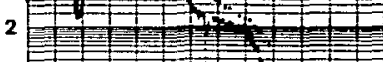

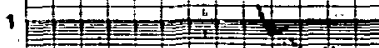

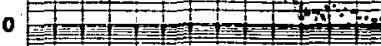

$-10_{0}=13$

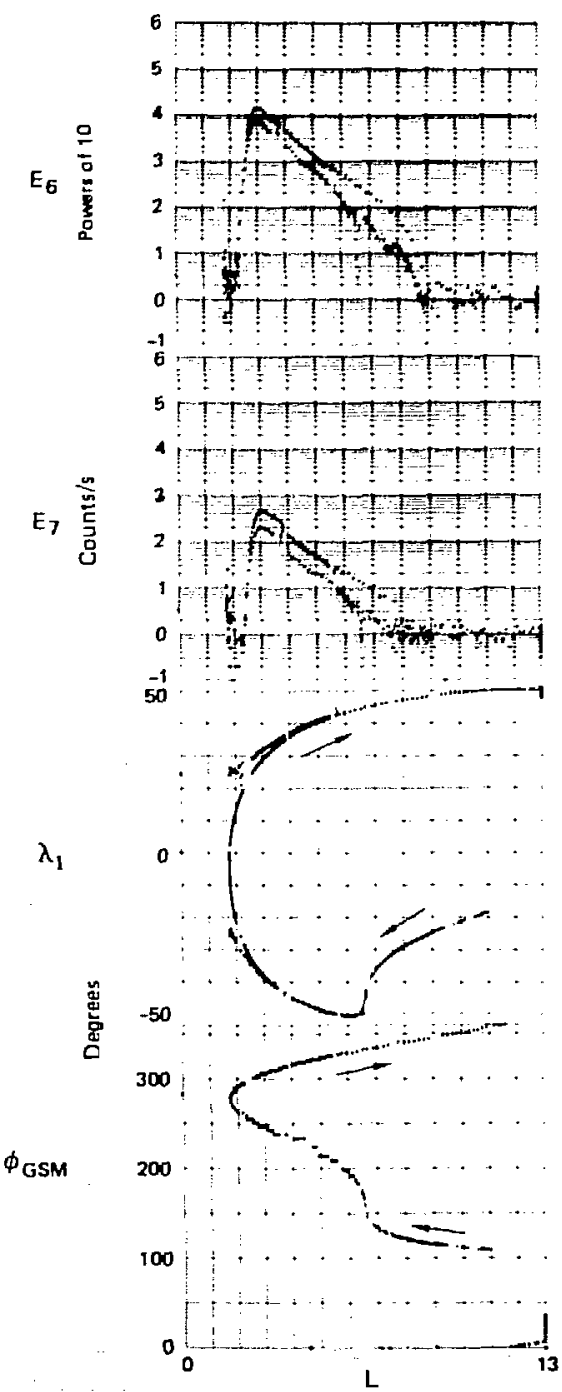

Fig. 9c. Radial $j_{\perp}-v s-L$ plots for Day 311, 1968. Note the marked enhancement and great radial extent of $E_{6}$ and $E_{7}$ fluxes over those observed on Day 306. 
EL. $\quad$ : : : : ${ }^{0}$

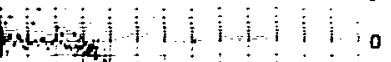

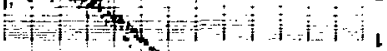
If $1+101$ ${ }^{9} \exists$

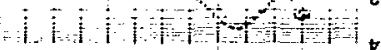
and

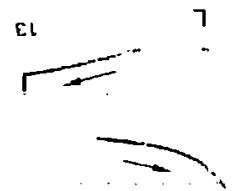

${ }_{0}$

001

$\operatorname{coz}$ wsפ $_{\phi}$

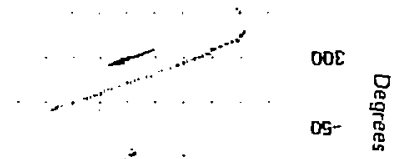

o

I

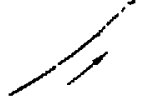

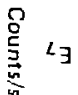

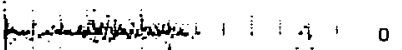

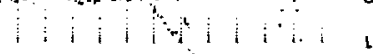
; 1 i 1 \%

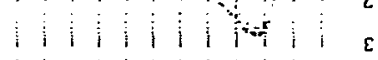

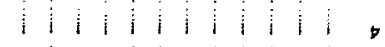

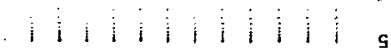

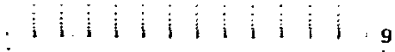

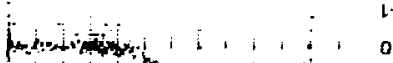
i i i i i i i i i i i 1

i i i i i i i.i

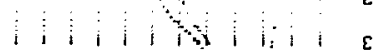

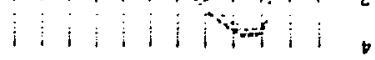

$1+1+1 \quad 1 \quad j \quad j \quad 1 \quad s$

i i i i i i i i i

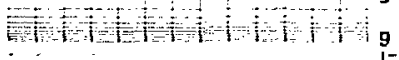
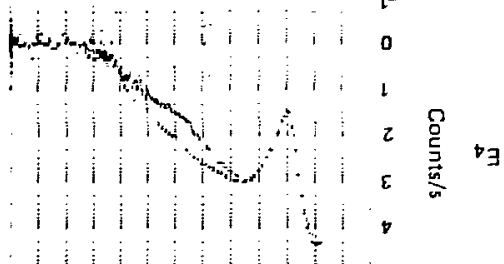

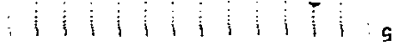

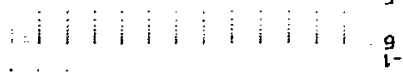
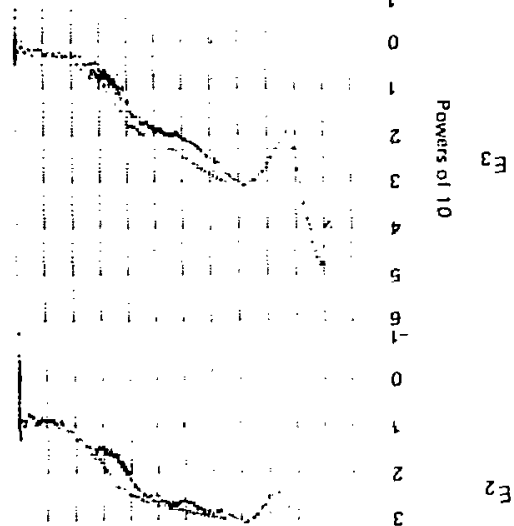

$\varepsilon$

23

13 
requator. The necessary pitch-angle corrections can be made using results we fur isentil below.

Figure 9c shows data acquired on Day 311. Comparison of the fluxes between Days 306 and 311 , especially in the regions $L=2$ to 4 , reves s that. even the highest-energy electrons are detected at great distances $(L=8)$ on this day. Figure 9d shows data accquired on Day 319. These data ref iert the changes that continue to occur post storm and clearly show the evolution of the slot region.

PITCH-ANGLE DISTRIBUTIONS

Fiqures $10 a-c$ show diagnostic PAD plots of $E_{1}-E_{6}$ data for 0 ay 142 , 1968. It was rare to find extended periods when Ogo 5 was close enough to the magnetic. Atnr for $L=3$ to 6 for good $P A D$ determinations (we need $\lambda_{I} \leq 10^{\circ}$ ). Notable examples of ' - ratisfying these constraints were obtained on ;ays 69 and 142 when 0 go 5 was inbound at -1000 LT and 0700 LT, respectively. We thought the Day 142 data were especially interesting; the lower energy distributions $\left(E_{1}-E_{3}\right)$ often show an asymmetry; if real, this effect means that there was a net current of electrons along the field line. Although such effects are often seen near dawn and dusk, we cannot rule out that partial shadowing by the solar panel did not occur at some point in the scan of the spectrometer aperture. However, there are other effects in the data that are quite real, and we focus our attention on them.

To fully appreciate these effects, one needs to be familiar with the calculations of Lyons and Thorne [1972], Lyons, Thorne, and Kennel [1972] and Lyons and Thorne [1973]. These calculations analyze the electron interactions with whistler-mode radiation for the cyclotron harmonics (including higher-order harmonics) and the Landau interaction. The rise in flux at $40-50^{\circ}$ pitch angles for $E_{1}-E_{3}$ results from the Landau interaction. We feel it quite worthwhile that the reader scan the data in $L$ for a given energy to observe how the PAD's change. [For further understanding of the data, see Lyons, Thorne, and Kenne1, 1972; Lyons and Thorne, 1973; Lyons and Williams, $1975]$.

In Figs. 1la-c, we show a second example of diagnostic PAD's. Here Ogo 5 was inbound at $~ 2000$ LT on an especially quiet day. At the more extended distances, the PAD's are largely depleted in flux near $90^{\circ}$ and peaked at $30^{\circ}$ pitch angles. Such PAD's are called butterfly PAD's, reflecting their 

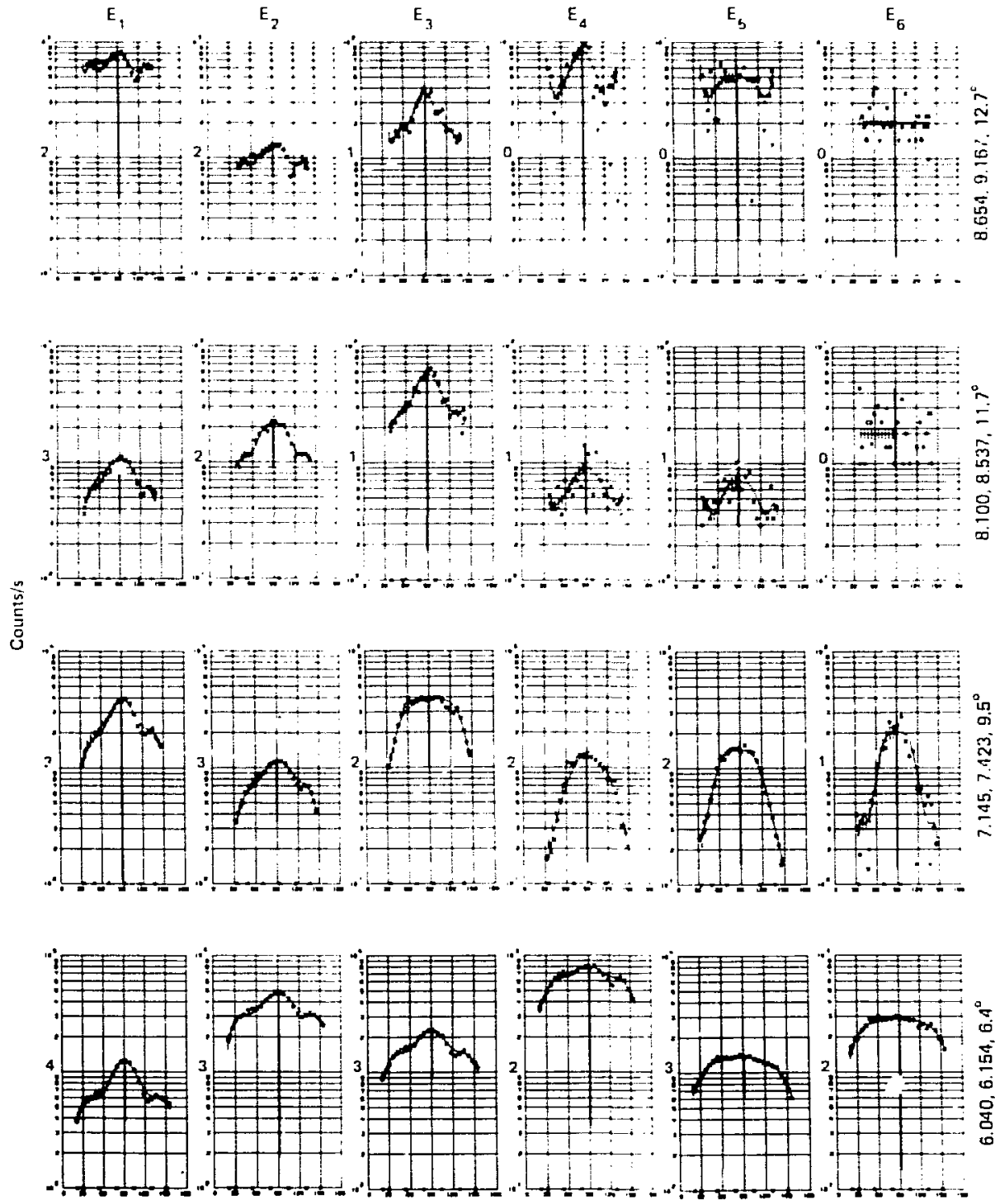

Pitch angles $0-180^{\circ}$

Fig. 10a. Diagnostic PAD's for Day 142, 1968. R, L, and $\lambda_{m}$ are given along the right side: of the plats; $\phi G S M=275-285^{\circ}$. The energy channel is given at the top of each set of PAD's. Fluxes ere in $\mathrm{c} / \mathrm{s}$. 

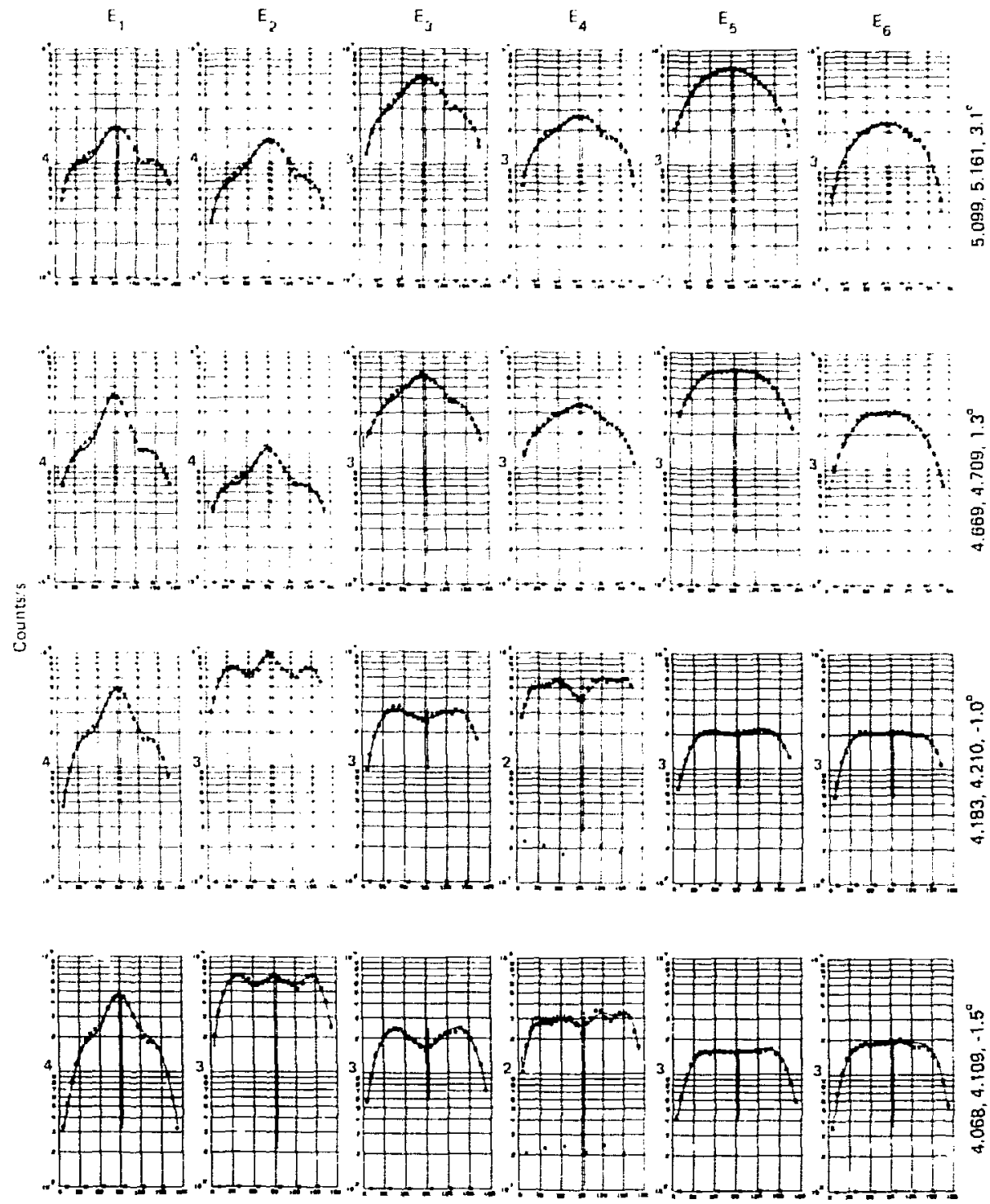

Pitch angles $0-180^{\circ}$

Fig. 10b. Diagnostic PAD's for Day 142, 1968. $R, L$, and $\lambda_{m}$ are given to the right of the plots; $\Phi G S M=287-293^{\circ}$. This is a continuation of Fig. 10 a. 

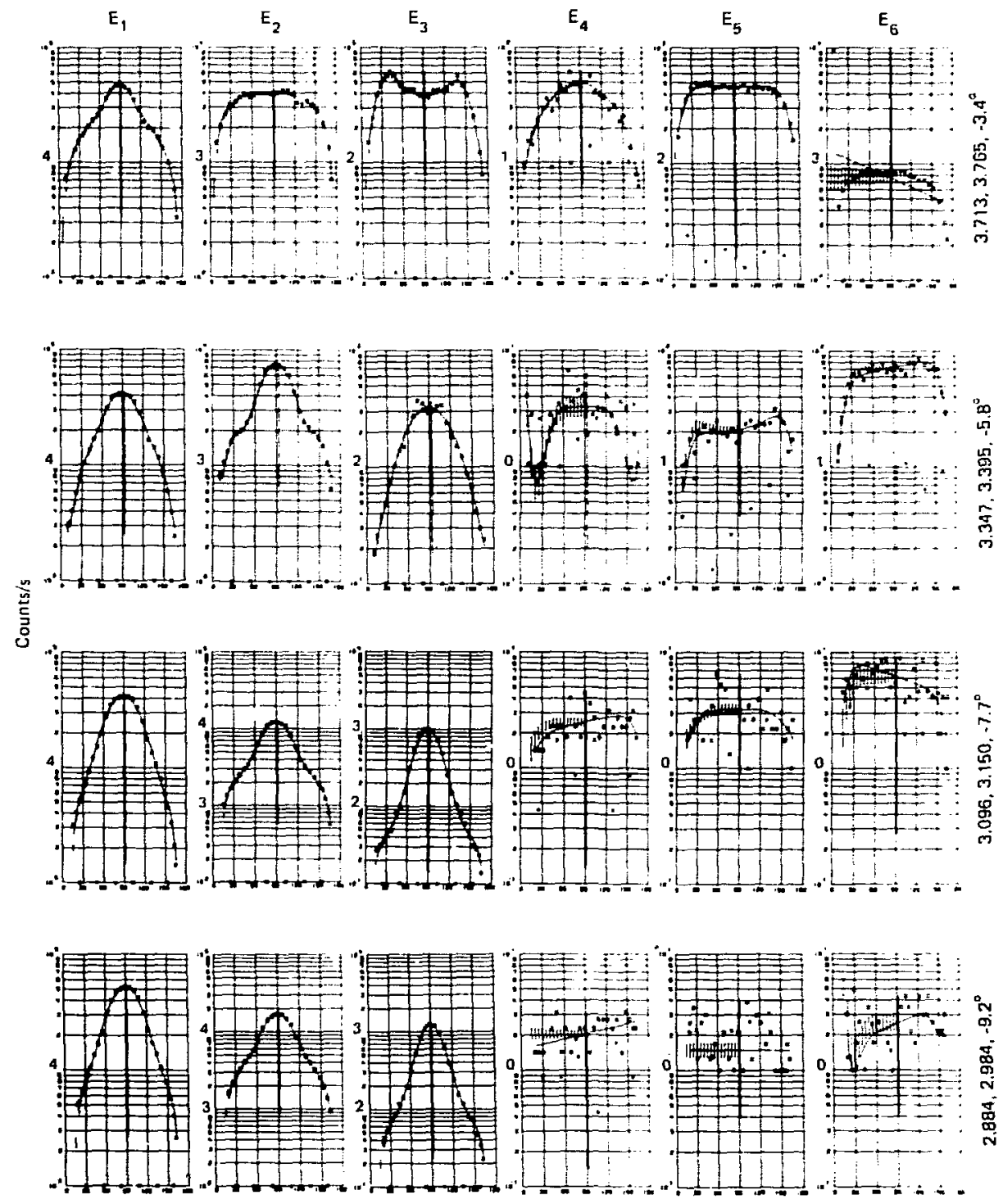

Pitch angle $0-180^{\circ}$

Fig. 10c. Diagnostic PAD's for Day 142, 1968. $R, L$, and $\lambda_{m}$ are given to the right of the plots; $\phi G S M=296-301^{\circ}$. This is a continuation of Figs. 10ab. 


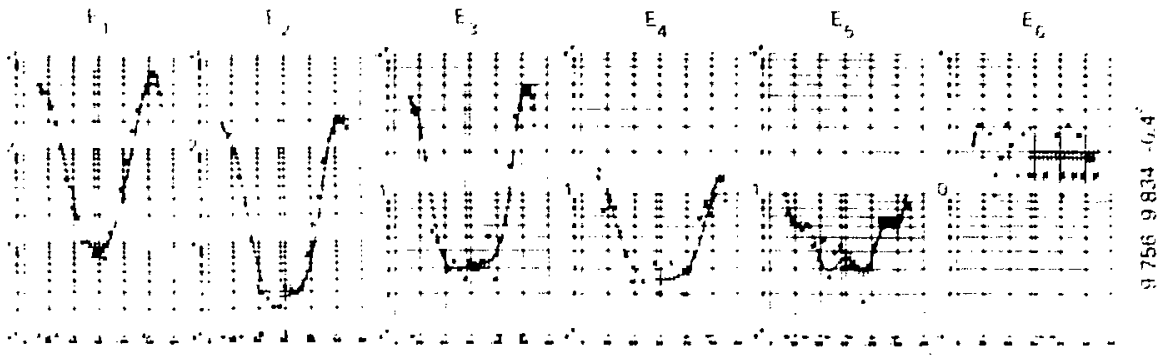

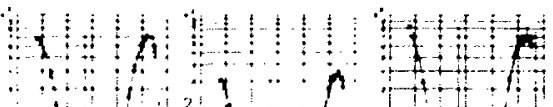

$\therefore$ a n

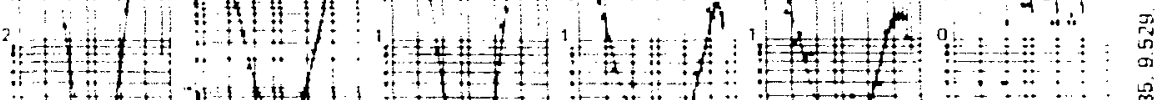

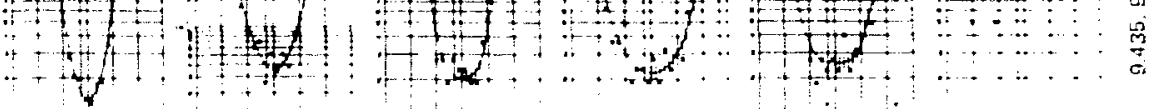
с
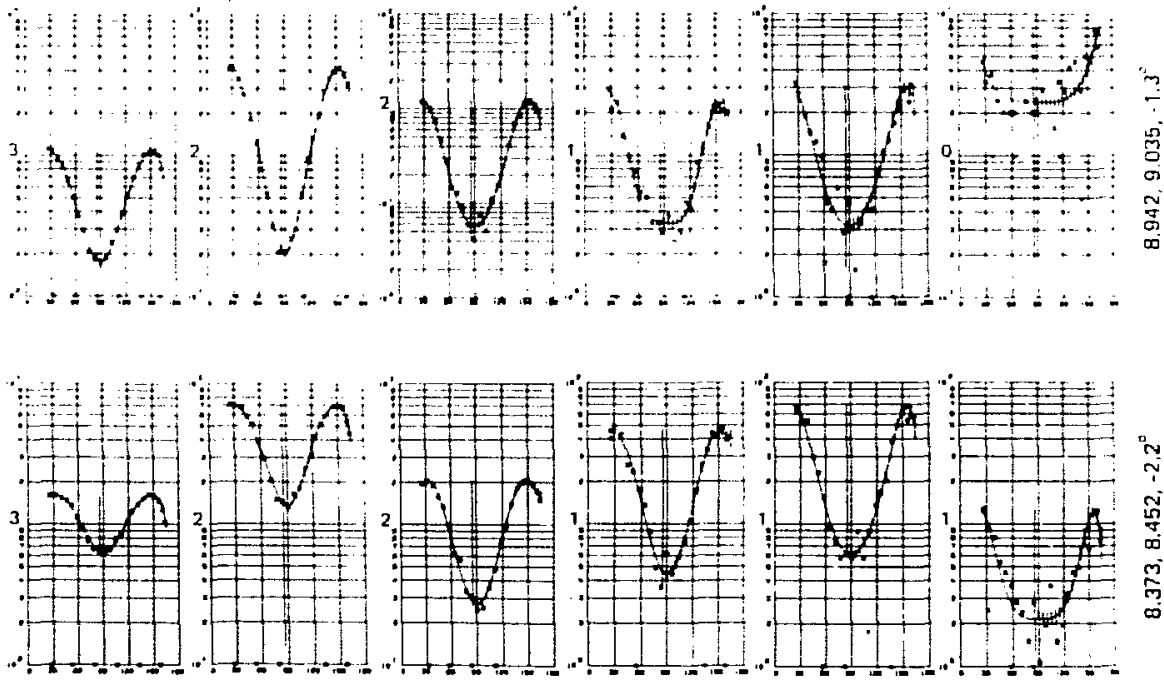

Pitch angles $0-180^{\circ}$

Fig. 11a. Diagnostic PAD's for Day 295, 1968. $R, L$, and $\lambda_{m}$ are given to the right of the plots; $\phi G S M=124-178^{\circ}$. 

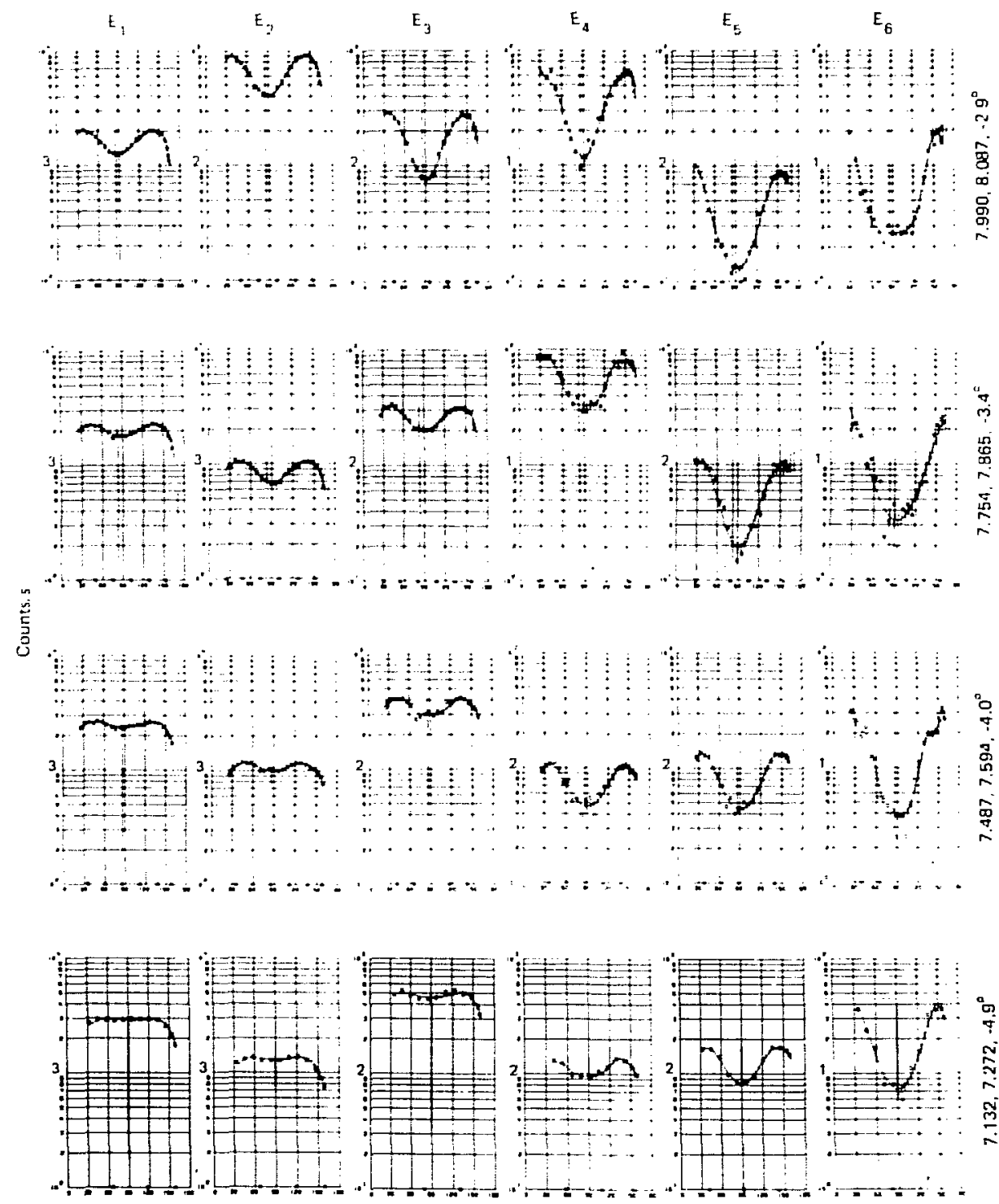

Pitch angles $0-180^{\circ}$

Fig. 11b. Diagnostic PAD's for Day 295, 1968. R, L, and $\lambda_{m}$ are given to the right of the plots; $\phi G S M=128-130^{\circ}$. This is a continuation of Fig. 11 a. 
$\begin{array}{lllllll}E_{1} & E_{2} & E_{3} & E_{4} & E_{5} & E_{6}\end{array}$
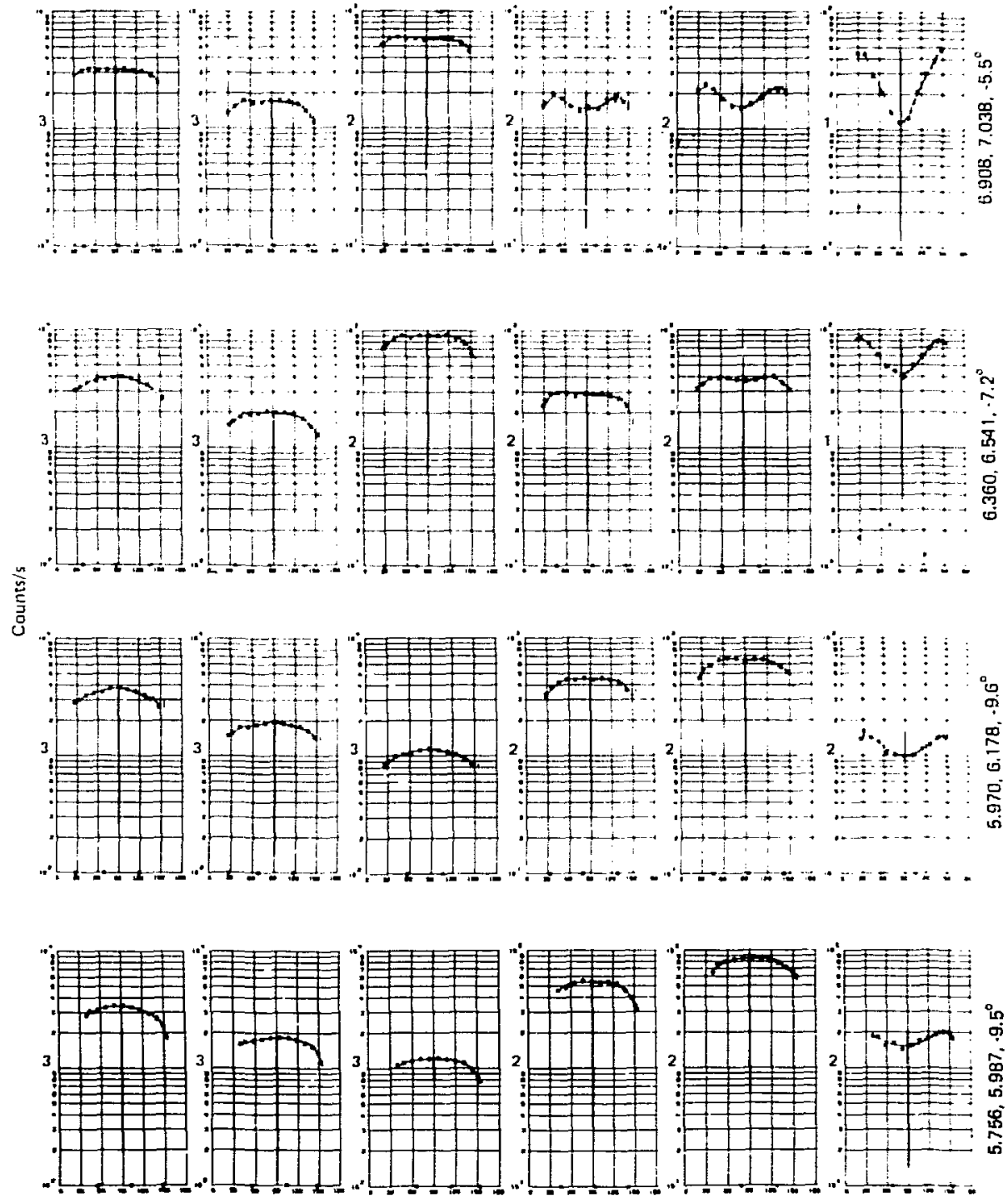

Pitch angles $0-180^{\circ}$

Fig. 11c. Diagnostic PAD's for Day 295, 1968. $R, L$, and $\lambda_{m}$ are given to the right of the plots; $\phi_{G S M}=131-136^{\circ}$. This is a continuation of Figs. 11ab. 
shape in a polar plot. Nearer the earth, the butterfly PAD is lost, and the distribution changes gradually to the normal loss-cone distribution (note that the butterfy $P A D$ is also a loss-cone distribution). The appearance of the butterfly PAD in the night-time magnetosphere and in the early afternoon magnetosphere at extended distances is due to shell splitting. Such PAD's have been thoroughly documented as part of a study of the PAD's of energetic electrons in the equatorial regions of the magnetosphere at all local times [West et al., 1973bc; West and Buck, 1974; West, 1978].

In Figs. 12a- $f$, we show PAD's normalized in intensity at $90^{\circ}$ for $L=3$, $3.5,4.0,4.5,5.0$, and 6.0 that resulted from using the J0B06 option of the

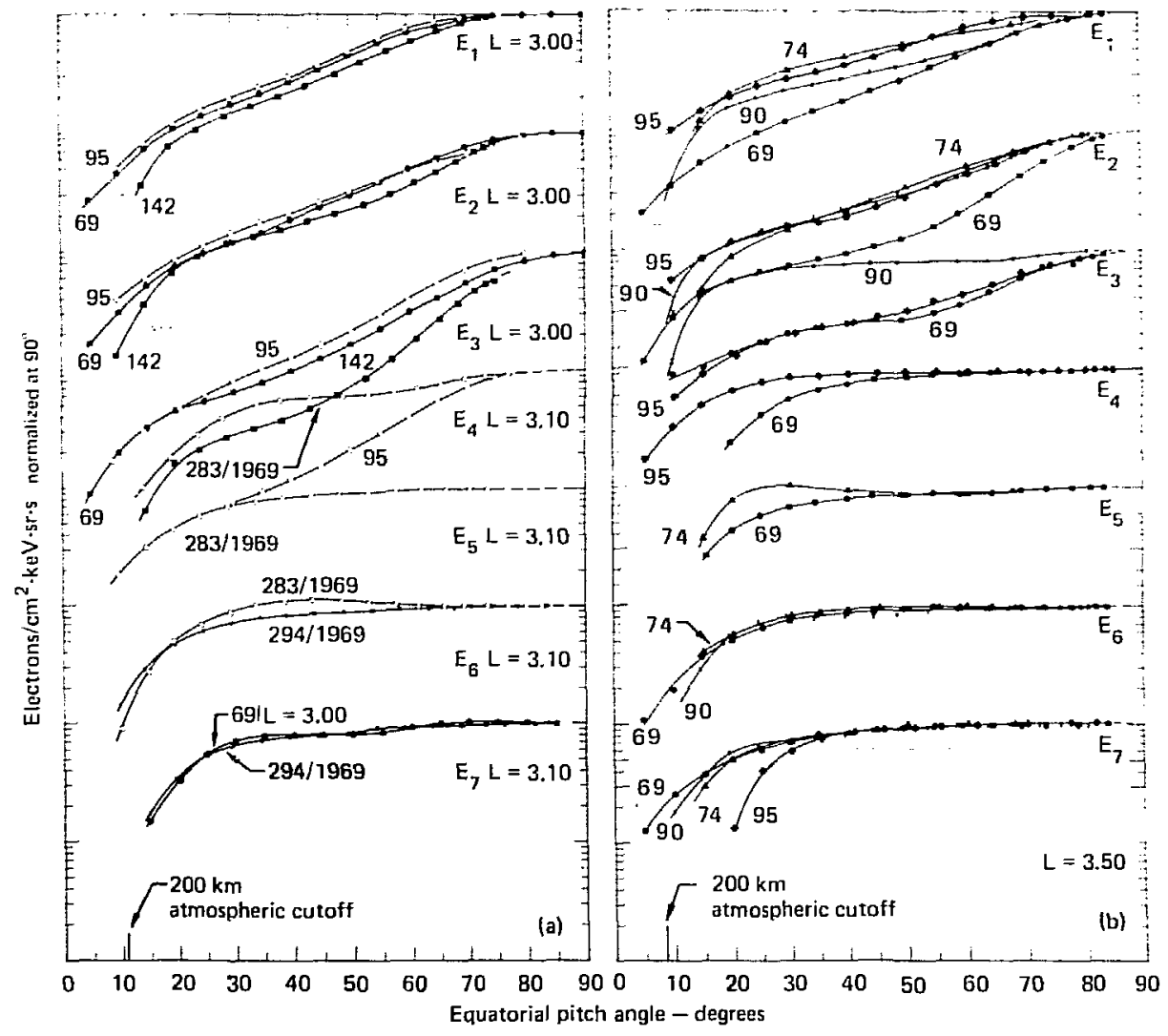

Fig. 12ab. Pitch-angle distributions for $(a) L=3.00$ and 3.10 and $(b) L=$ 3.50. See Table 2 for the conditions of data acquisition. The data in this and subsequent figures were not corrected for the finite resolution of the spectrometer; such a correction is quite important in the region of the loss cone. 


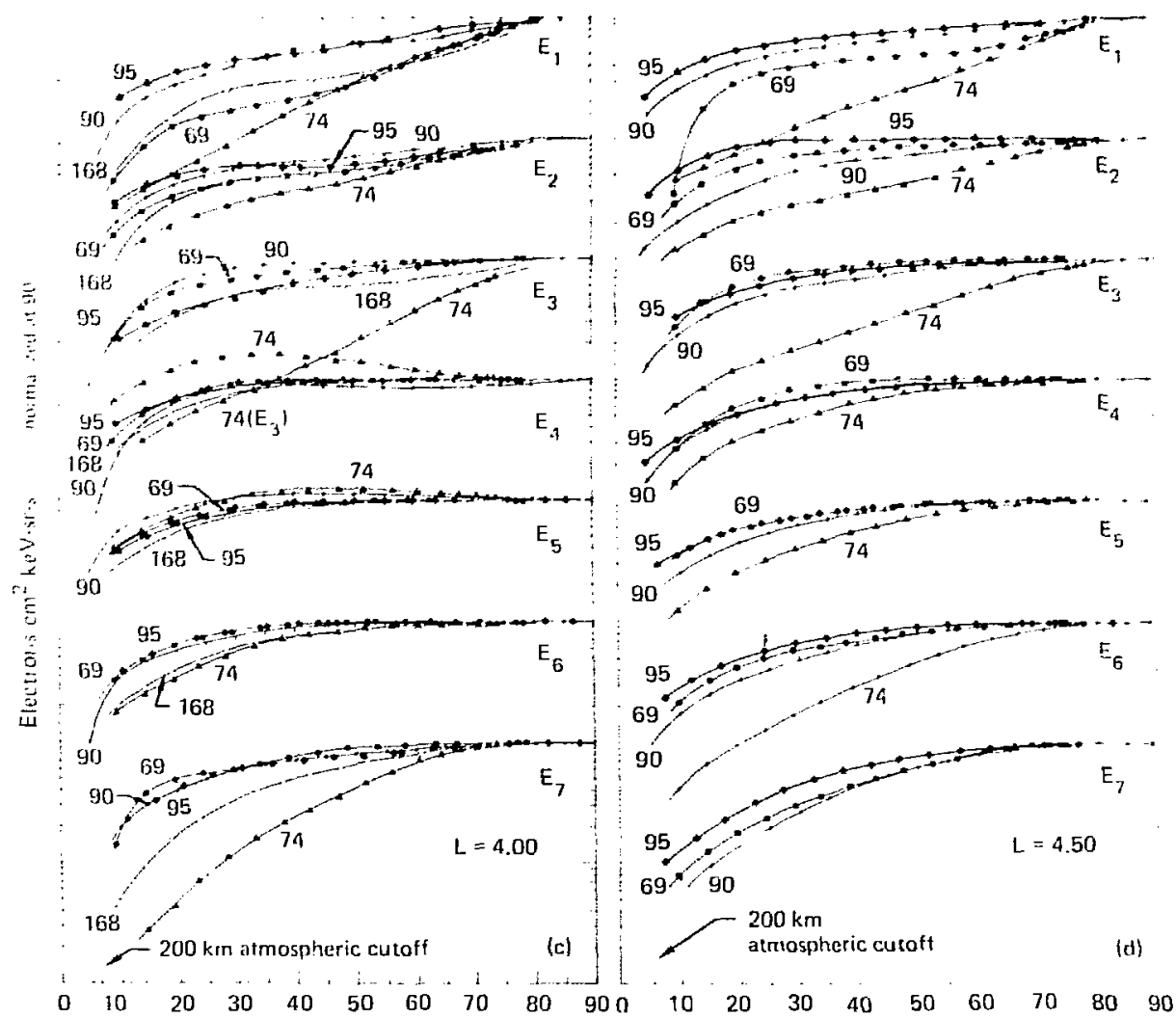

Equatorial pitch angle - degrees

Fig. 12cd. Pitch-angle distributions for (c) $L=4.00$ and (d) $L=4.50$.

BPLTM code. In each case, the results of two or more PAD's were combined to produce the plotted results. For Day-69 data, four PAD's were fitted at constant angle by least squares. In general, however, such a refinement was not particularly warranted, but it was important to average two or more PAD's graphically to ensure that no artifact in the analysis was included. In using the data, it should be noted that finite-resolution corrections were not applied to the data; in the region of the loss cone, the intensities should roll off to zero.

The major use for the PAD's in this study was to correct the $j_{\perp}$ data to a common $\alpha_{0}$ (equatorial pitch-angle). The $j_{\perp}$ data, having been acquired at a range of magnetic activity levels and local times, should be corrected by a fairly diverse set of PAD's. Our data set contains a vast amount of 


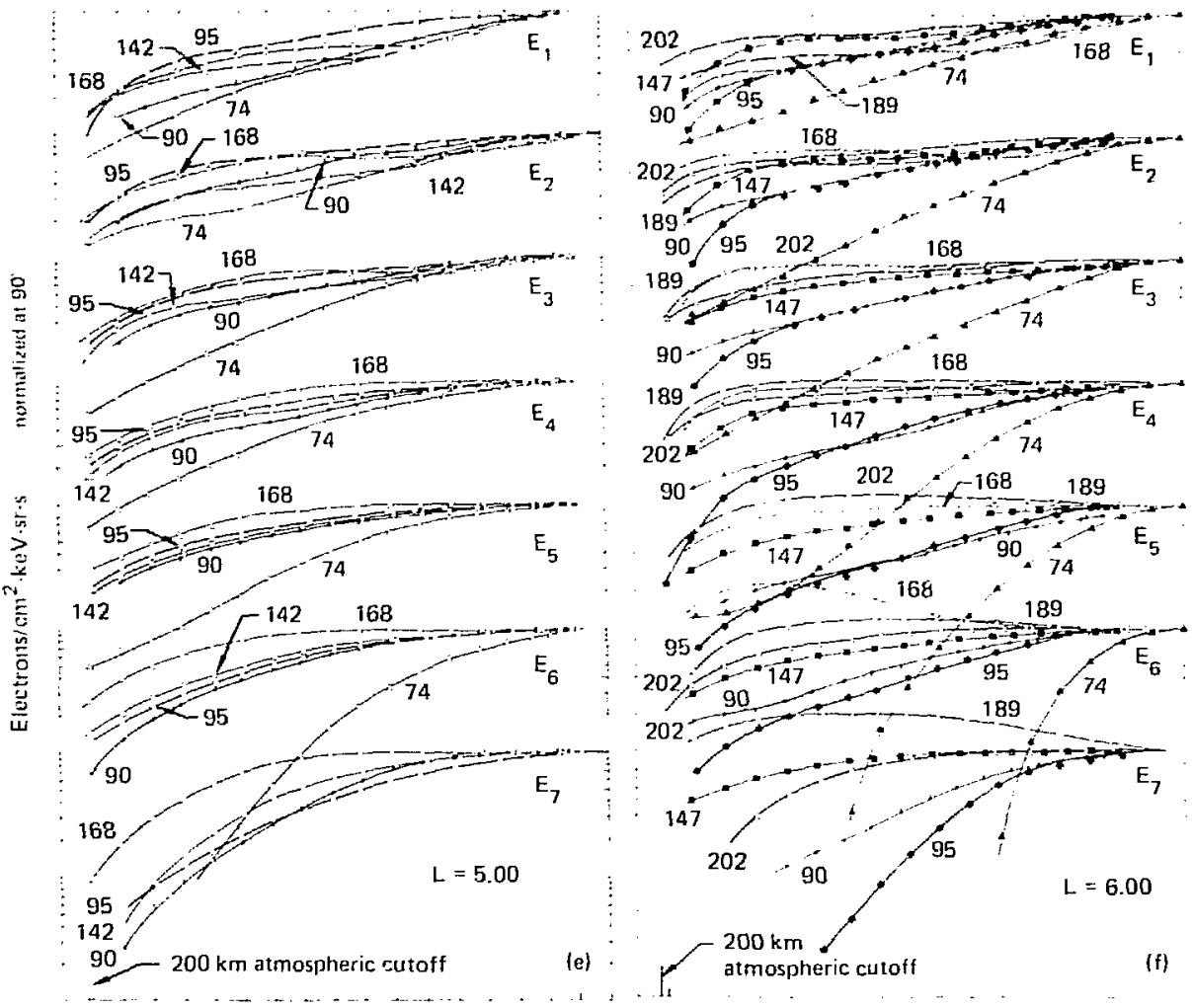

Equatorial pitch an.je - degrees

Fig. 12ef. Pitch-angle distributions for $(e) L=5.00$ and $(f) L=6.00$.

pitch-angle information, but it is not always simple to find exactly what we want in the data in terms of energy, local time, and magnetic activity. In this respect, we have of ten used data acquired during the early days of Ogo 5 when it was inbound in the 0600-1200 LT sector; at these times, good equatorial coverage at $L \simeq 3$ was obtained. Also, good equatorial coverage at $L=3$ was available in 1969 when perigee had risen. Equatorial coverage at $L \simeq 5$ to 6 was available throughout the year. For the present purposes (next section), we use Days 69 and 95 data at $L=3.0$ to 4.5 and Days 90 and 95 data for $L=5.0$ and 6.0. We are interested in making pitch-angle corrections for moderately quiet periods after the storms when the magnetosphere had settled down. 
Ill an attempt to understand the PAD's, we prepared Table 2 showing Dst. and $A E$ at the time of the PAD measurements and back up to 7 hours earlier. For L 6.0, shell-splitting effects, although apparent, are not dominant. The major effects seem due to magnetic activity and seem to reflect the efferts of substorm artivity (possibly on the timescale of a drift period). The major deviation from the norm is for the Day-74 data, and these data were acquired during a major substorm.

It is interesting that the PAD's for $L=3-5$ show the marked effects of the Landau interaction for equatorial pitch-angles $>45^{\circ}$ for a disturbed day (Day 142) as well as for a very quiet day (Day 69). At the lower L-shell; inside $L=2.5$, the effect is generally absent (see West and Buck, 1973t), i.e., the energy dependence is much reduced. For this reason, the plot (Fig. 13) for Day 135, 1969, which shows data obtained during the main phase of a magnetic storm (Dst $=-120 \gamma$ ), is of interest; the effects of the Landau interaction are clearly evident for the $E_{4}$ data. Possibly it occurred for the $E_{5}$ data as well, but the counting rates in the PAD were not above background at $10 w$ angles. Data obtained on Day 95 are shown for comparison and are used later in our data analysis.

\section{TIME-DECAY DATA}

The $j_{1}$ fluxes for Days 147-223, 1968, were obtained and plotted for $L=3$, $3.5,4,4.5,5$, and 6 in Figs. $14 \mathrm{a}-\mathrm{g}$. The data were acquired at $\lambda_{\mathrm{I}}$ varying from 10 to $48^{\circ}$ with most of the data in the $20-30^{\circ}$ range. In principle, the data should be corrected to a common pitch angle and this we have Jone for the most sensitive data. Rather than correcting the data to an equatorial $90^{\circ}$ pitch angle, we chose to use that value of $\alpha_{0}$ for which $j\left(\alpha_{0}\right)$ is independent of local time. Schulz [1972] has found that for particles having an equatorial pitch angle of $45.7^{\circ}$, the $\operatorname{trace}\left(\lambda_{I}=22.7^{\circ}\right)$ of the mirroring particles is a circle, and $\mathrm{j}\left(45.7^{\circ}\right)$ is constant under conditions that conserve the adiabatic invariants. Because this angle minimizes some of the problems of L-shell splitting and field models, it seemed especially appropriate. Actually, however, the regions in which we have made the pitch-angle corrections, i.e., $L=3-4.5$, are not subject to very strong shell-splitting effects. The data thus corrected are evident in Figs. 14a-g. Because of the choice of $\alpha_{0}=45.7^{\circ}$, the average of the data has not changed significantly from that of the uncorrected data. For the lower energy 



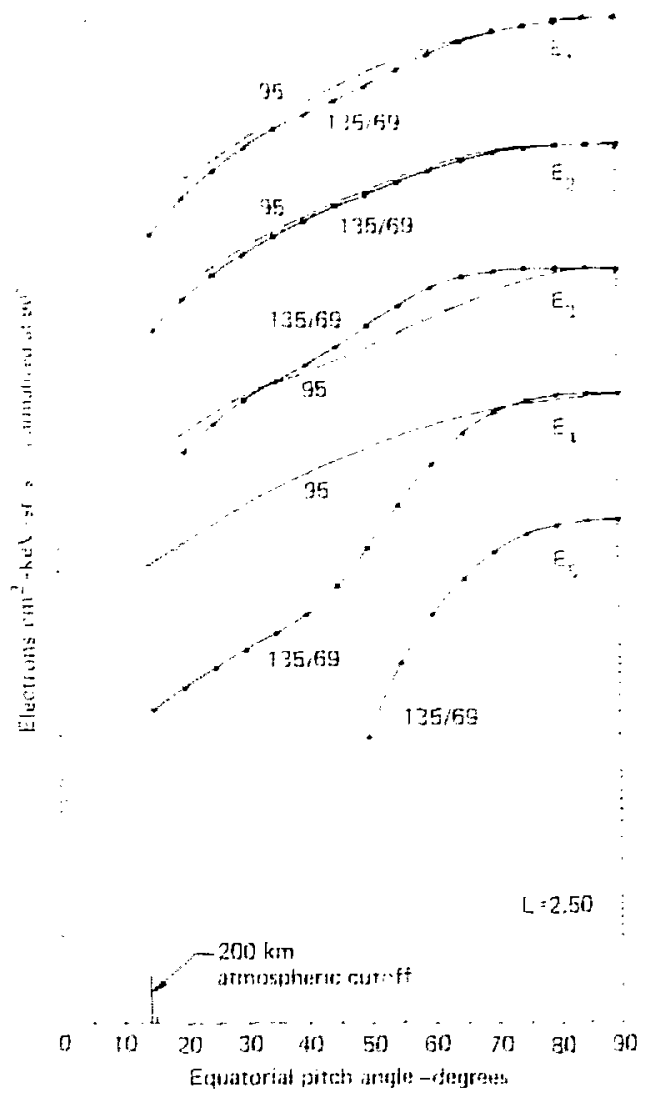

Fig. 13. Pitch-angle distributions for $L=2.5$. The data for Day 135,1369 , are for a magnetically disturbed day, whence the appearance of the effects from the Landau interaction in the $E_{a}$ data. In contrast, the data abtained on Day 95 are representative of a quiet day.

Shannels, however, the exponential decays of the data have become very much better defined.

At the bottom of each $j_{1}$-vs-time plot, we have included daily averages of the auroral electrojet index ( $A E$ ) and Dst. The AE, being based upon the deviations of the auroral-zone magnetrmeter data (width of the envelope of the total deviations in $\mathrm{H}$ at selected stations), is a measure of substorm activity and is thus indicative of the short-term injections. Dst, on the other hand, is based on deviations in $\mathrm{H}$ of ecuatorial magnetameter data and as such is sensitive to the diamagnetic eff scts of the ring current of trapped particles, 

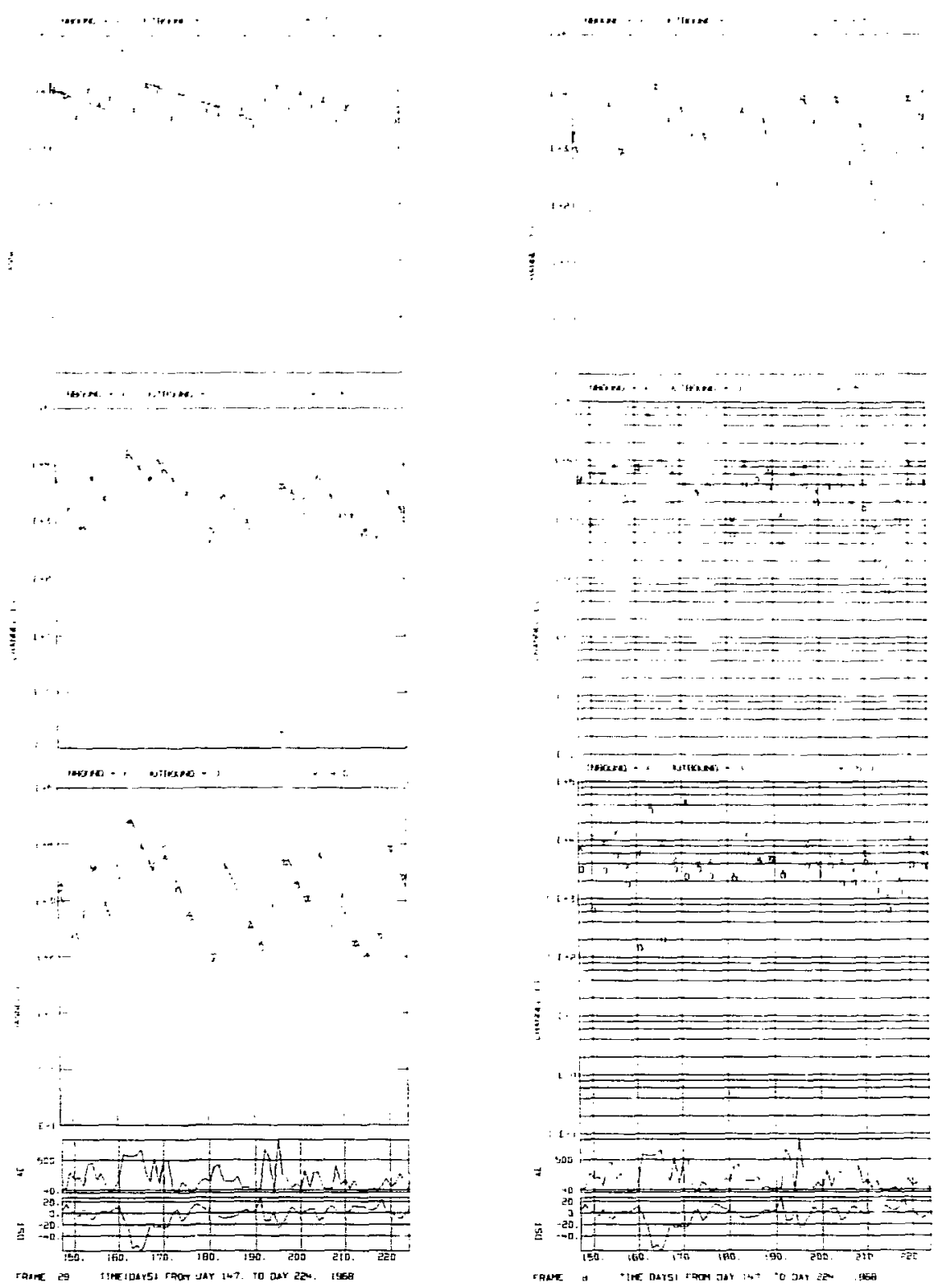

Fig. 14a. Flux-vs-time plots of the $E_{1}(79 \mathrm{keV})$ counting rates for Days $147-224,1968$. The data without grid ines are correr.ted to $45.7^{\circ}$ equatorial pitch angle; the lines indicating decay are all of the same slope. AE and Dst are daily averages. Note that the data are plotted as count rate minus background plus $1 \mathrm{c} / \mathrm{s}$. 

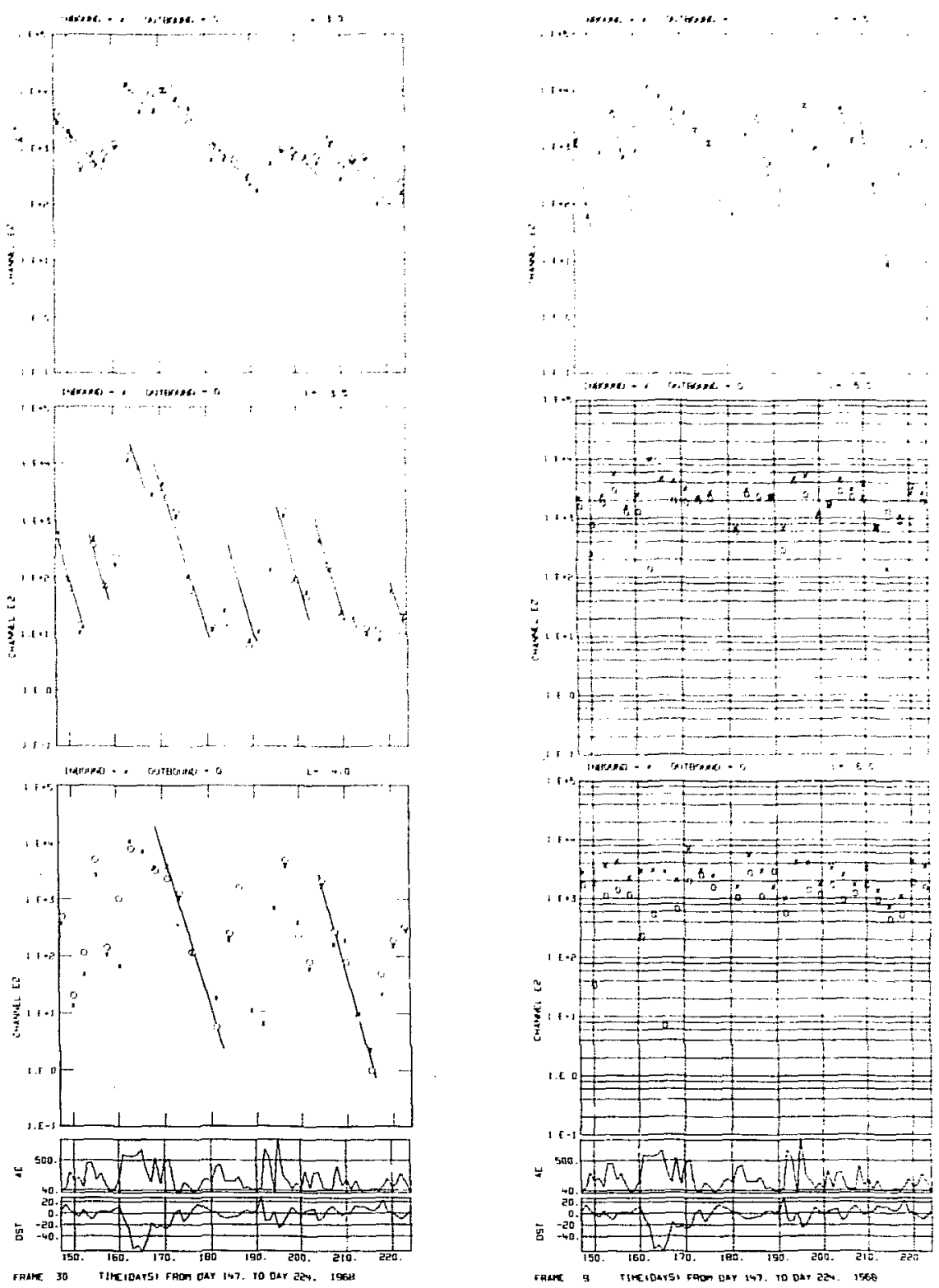

Fig. 14b. Flux-vs-time plots of the $E_{2}$ (158 keV) counting rates for Day $147-224,1968$. The data without grid Tines are corrected to $45.7^{\circ}$ equatorial pitch angle; the lines indicating decay are all of the same slope. 

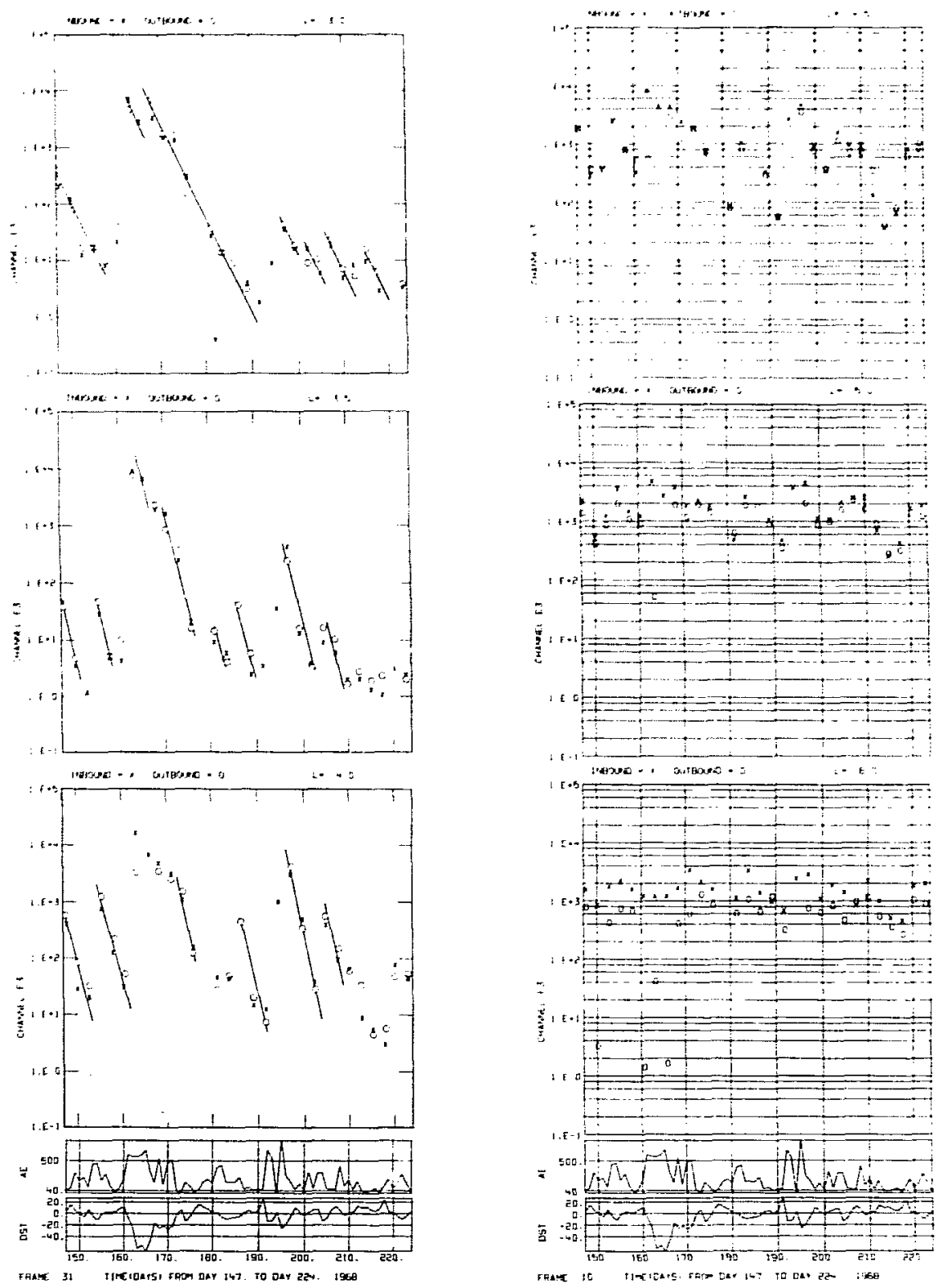

Fig. 14c. Flux-vs-time plots of the $E_{3}(266 \mathrm{keV}$ ) counting rates for Days 147-224, 1968. The data without grid lines are carrected to $45.7^{\circ}$ equatorial pitch angie; the lines indicating decay are of the same slope. 

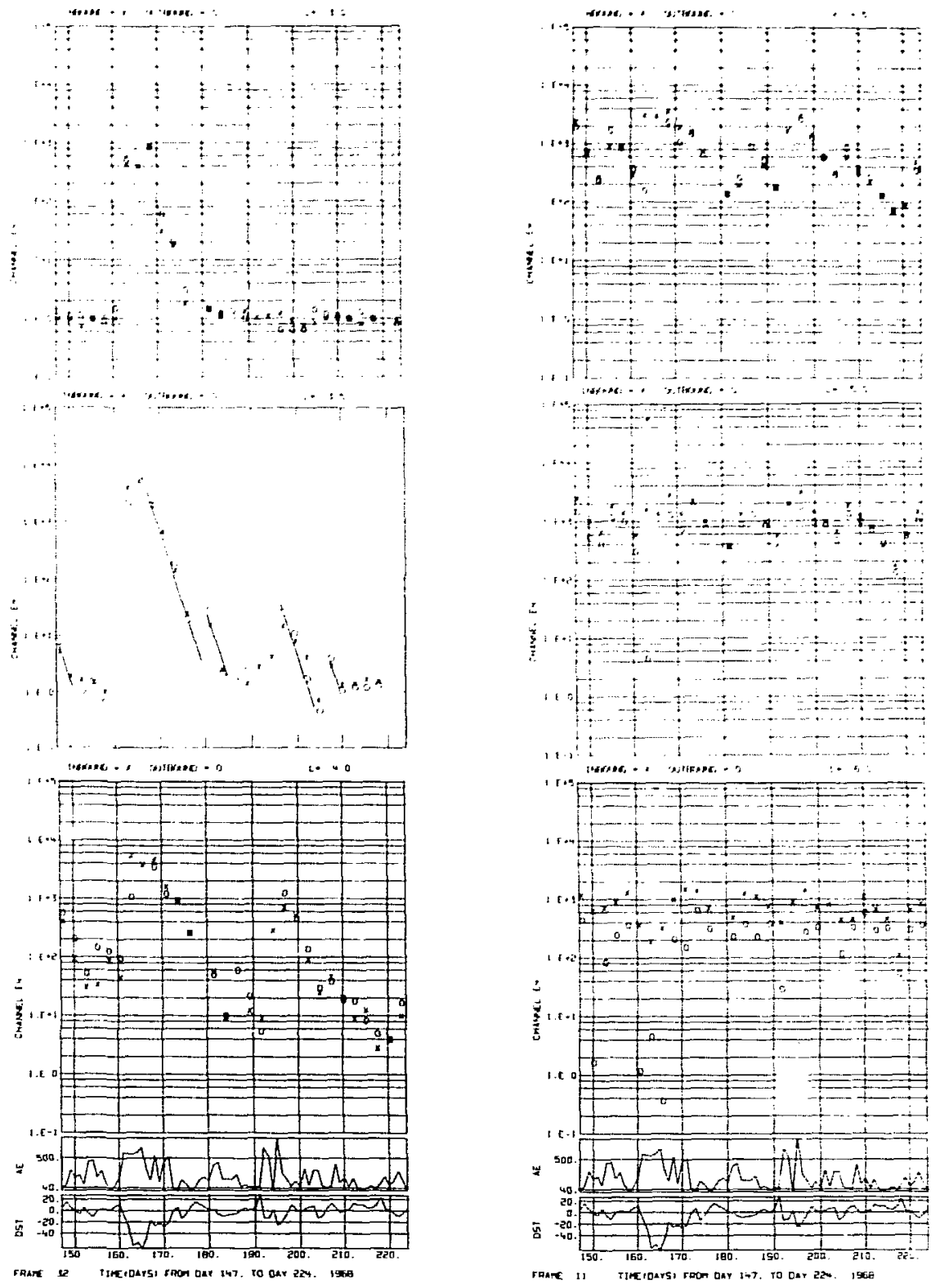

Fig. 14d. Flux-vs-time plots of the $E_{4}(478 \mathrm{keV})$ data for Days 147-224, 1968. The data without grid lines are corrected to $45.7^{\circ}$ equatorial pitch angle; the lines indicating decay are all of the same slope. 

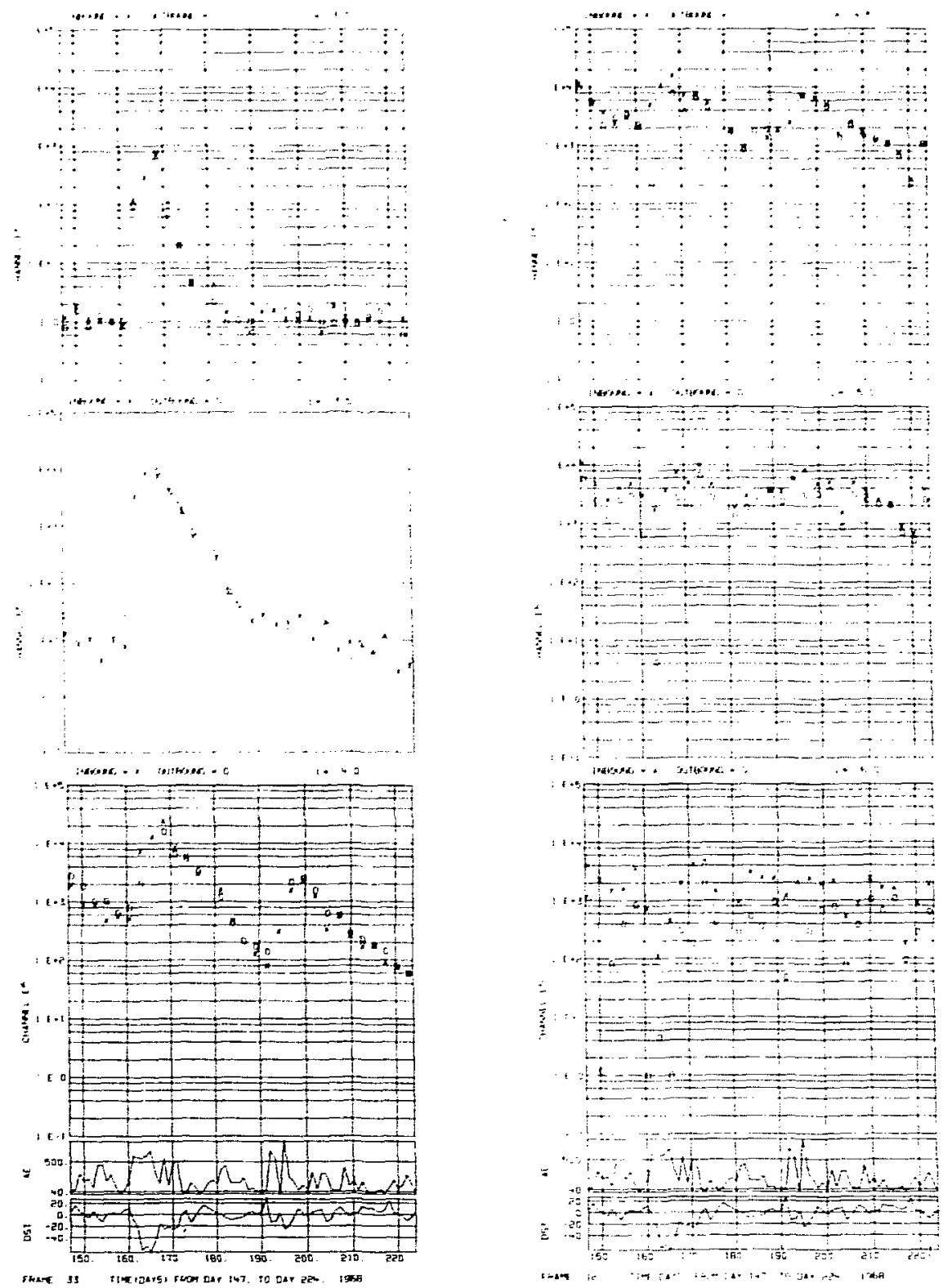

Fig. 14e. Flux-vs-time plots of the $E_{5}(822 \mathrm{keV})$ data for Days 147-224, 1960. The data without grid lines are corrected to $45.7^{\circ}$ equatorial pitch angle. 

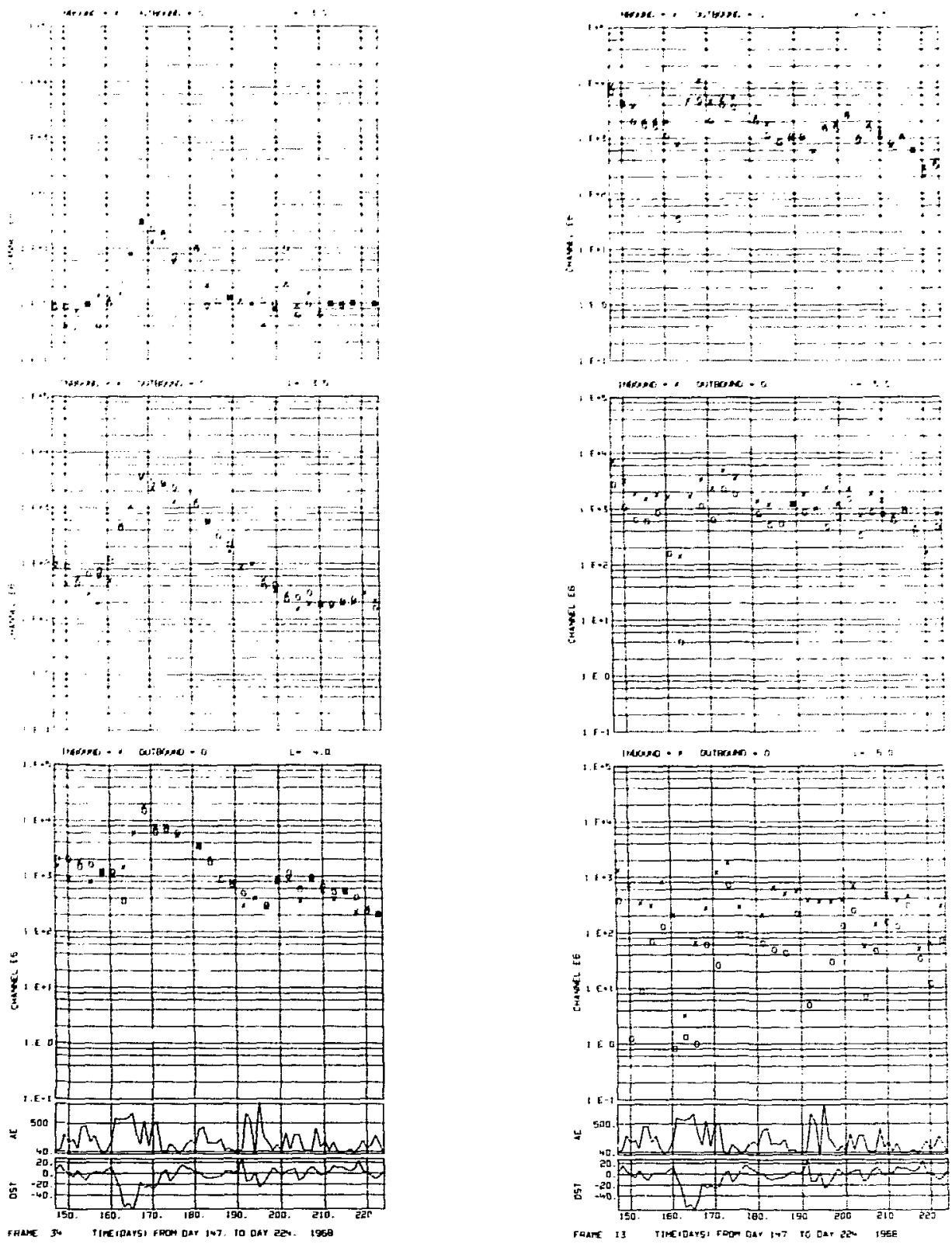

Fig. 14f. Flux-vs-time plots of the $E_{6}(1530 \mathrm{keV})$ data for Days 147-224, 1968. 

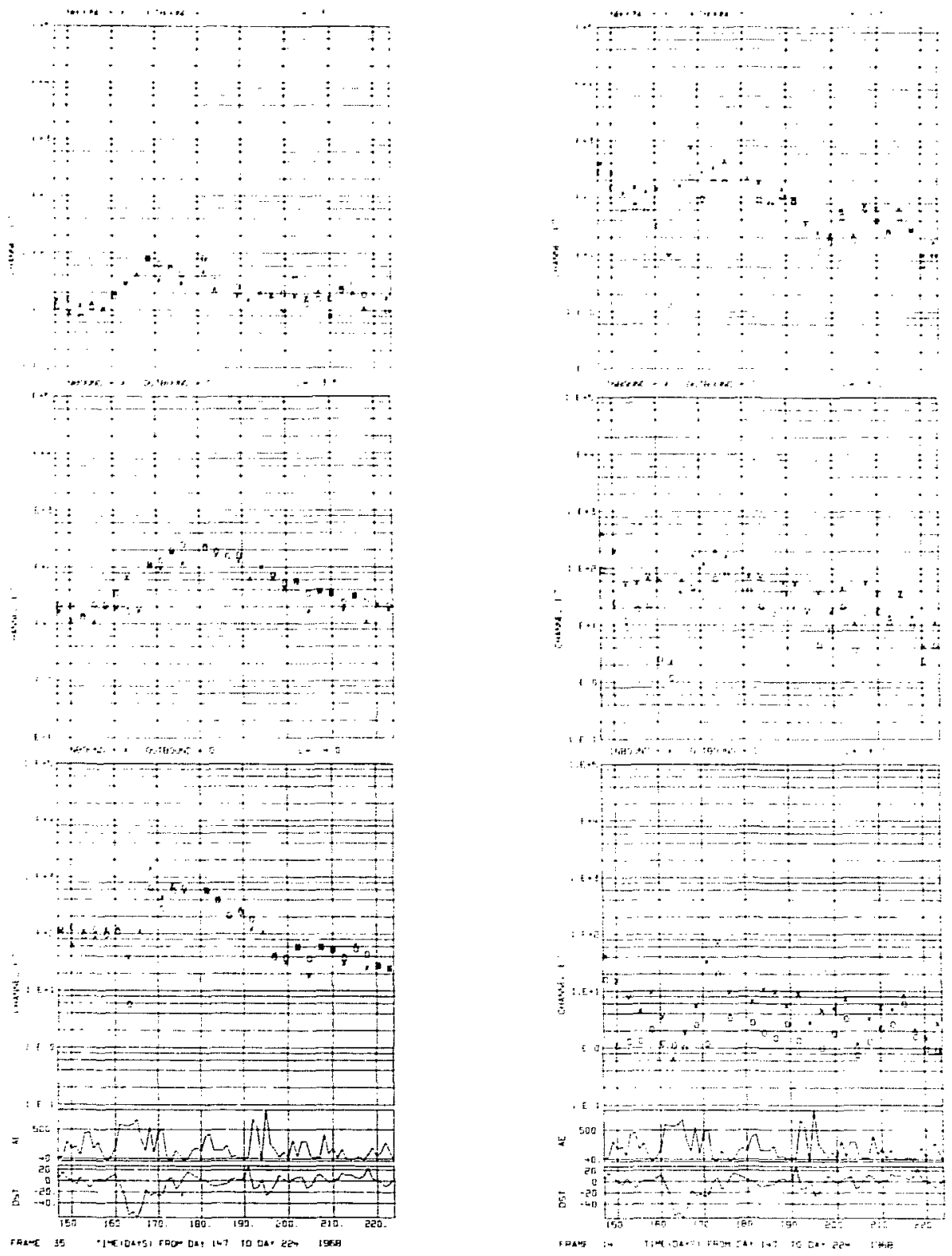

Fig. 14g. Flux-vs-time plots of the E7 (2820 keV) data for Days 147-224, 1968. 
which are enhanced most strongly during magnetic storms. It is probably possible to find regions in the data that correlate better with AE than Dst, but for the most part, the dependence is most closely correlated with the latter. The major injections occurred at Days 168, 173, 182, 208, and 216. The minor injections are most clearly visible at $L=4.5$ for the lower energy channels.

Before discussing the time decays, we present $j_{1}$-vs-time plots for the period associated with the October-Noveinber storms. Data for $L=4.0$ shown in Fig. 15 have been corrected to $45.7^{\circ}$ equatorial pitch-angles. We note rather marked correlation between the injected electron intensities and the negative excursions in Dst. (The positive values of Dst are usually attributed to compressions of the magnetosphere by enhancements of the solar wind dynamic pressure.) As was true for the June period, we find that the lower energy electrons, $E_{1}-E_{4}$, show a much lower threshold for injection than do the higher energy electrons, $E_{5}-E_{7}$. It is also noteworthy, as mentioned in discussing the radial plots, that a marked flux drop-out appears at the beginning of the storm in $E_{7}$ and $E_{6}$ and to some extent in the $E_{5}$ data. This drop-out is attributed to wave-particle interactions at the plasma pause, as suggested on a theoretical basis by Thorne and Kennel [1971]. An earlier example of these effects was observed by Vampola [1971] during disturbed times.

Together with the time-decay data of Fig. 15, we also present the $E_{6}$ data at $L=3.0$ in Fig. 16. The data were corrected to $45.7^{\circ}$ equatorial pitch-angle using PAD data acqu" $d$ on Days 283 and 294, 1969 (Fig. 17a). In Fig. 16, we note that exponential decay continued over a period of two months to background. (There were no electrons of this energy at $L=3.0$ before the storm.) Later, a radial-diffusion analysis indicates that for a fe'l weeks there was a weak inward flow of particles and that the decay was dominated by pitch-angle diffusion. The exponential decay suggests that (1) the decay was proportional to the number of $-1500-k e V$ electrons present, and (2) the wave spectrum producing the decay was remarkably constant with time. The waveparticle interaction is considered to be parasitic, i.e., the waves came from particles of very much lower energy than $1500 \mathrm{keV}$. It would appear that those fluxes were fairly constant in time, possibly being at the wave self-excitation limit [Kennel and Petsheck, 1966]. 
The June data were analyzed for time decay, and the results are presented in Table 3. Results labeled with an asterisk were pitch-angle corrected, but those that were not corrected are a consistent part of the pattern. At the higher $L$-shells ( $L=4.5$ and greater), some of the decay rates are based on only a few data points and hence are of reduced accuracy. It is interesting that the rapid decays for the $L=3.0$ and 4.0 data of October and November agree quite well with those of the June period. It is also significant that, after the initial fast decay, a slow decay was evident for $L=3.0$ (data not shown) and for $E_{4}$ and $E_{5}$; this slow decay indicates a transition to dominarce by radial diffusion.

Figure 17 shows a plot of mean-decay times vs $L$ for $E_{1}-E_{7}$. The data came primarily from the June study at $L>2.4$ but with a few points obtained from the October-November study. Regarding the separation of radial diffusion from pitch-angle diffusion, we note from Table 3 that for $E_{7}$ for the 0ctoberNovember storm, the decay time was 26 days; for June it was 8 days. Clearly the longer decay period resulted from radial diffusion.

Let us reexamine the June $j_{1}$-vs-time plots in Figs. $14 a-g$. The $E_{7}$ data at $L=3.5$ show a growth period of $\sim 15$ : days; at $L=3.0$, we find growth over 5-10 days. The $E_{6}$ data clearly show growth, $\leq 5$ days at $L=4.0, \sim 10$ days at $L=3.5$, and -5 days at $L=3.0$. The $E_{5}$ data also show evidence of growth before the ensuing decay. Conversely, it is interesting that the $E_{4}$ data show essentially no evidence of a growth period longer than the time between satellite passes ( 2.5 days) but show predominantly the total particle flux increase at the onset of the storm. Clearly, the data showing growth periods reflect the effects of radial diffusion. To just what extent we are seeing the effects of pitch-angle diffusion in the decay period or radial diffusion must come from a diffusion analysis as provided in the last section. However, it is our thesis that the most rapid decays that we see as we search through the various data sets are due predominantly to pitch-angle diffusion.

The rapid decay periods in the June data are all fairly consistent in value. The lines indicating decay as shown on the corrected curves of Figs. $14 a-g$ are the same on each curve and fit the data rather well. However, one feature in the $L=4.0$ November data of $F i g .15$ is not unierstood. In examining the $E_{1}-E_{4}$ data, we find evidence of continued injection during Days 305-330, and the decay periods around Days 280, 290, 340, and 360 are 


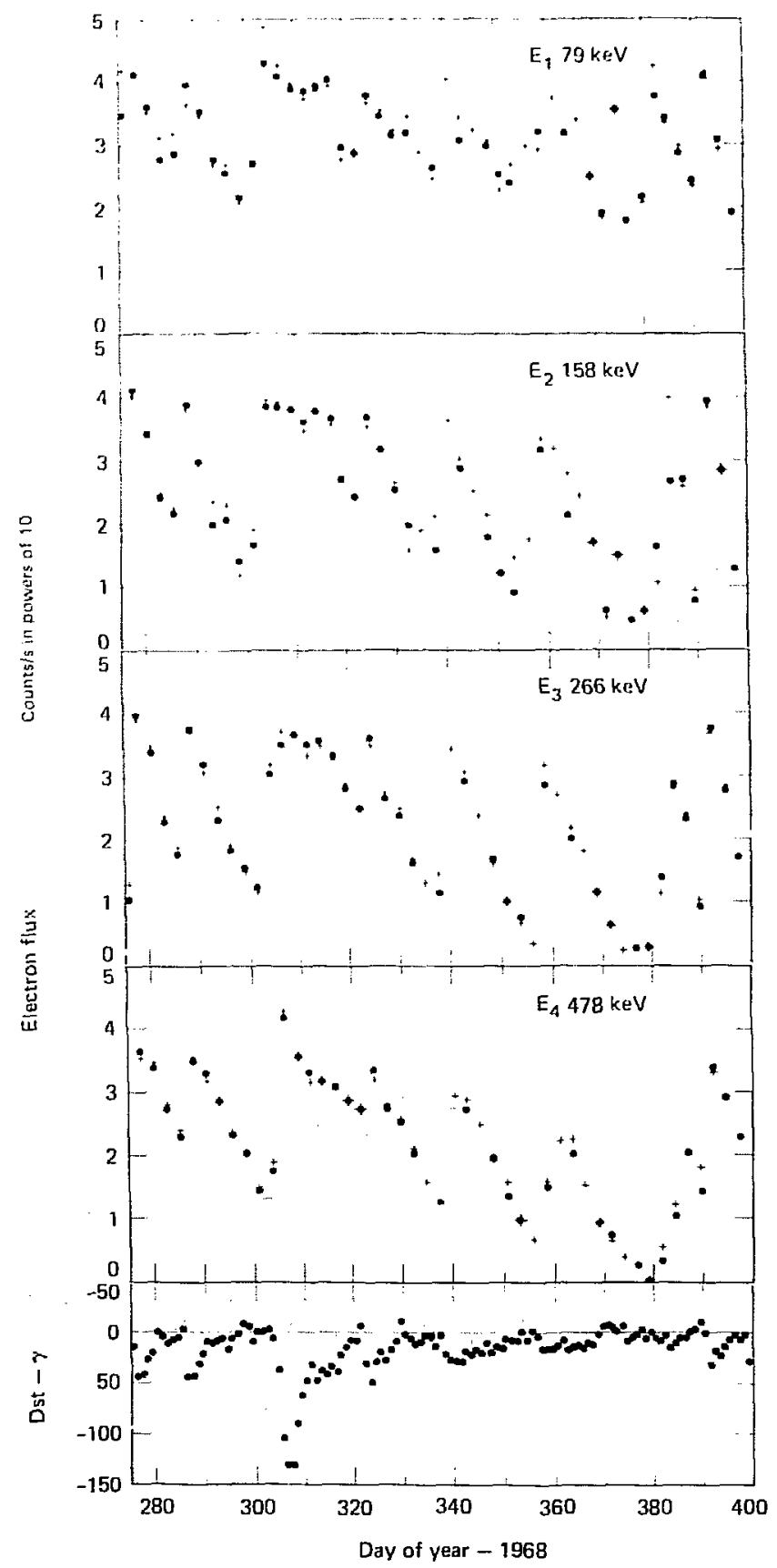




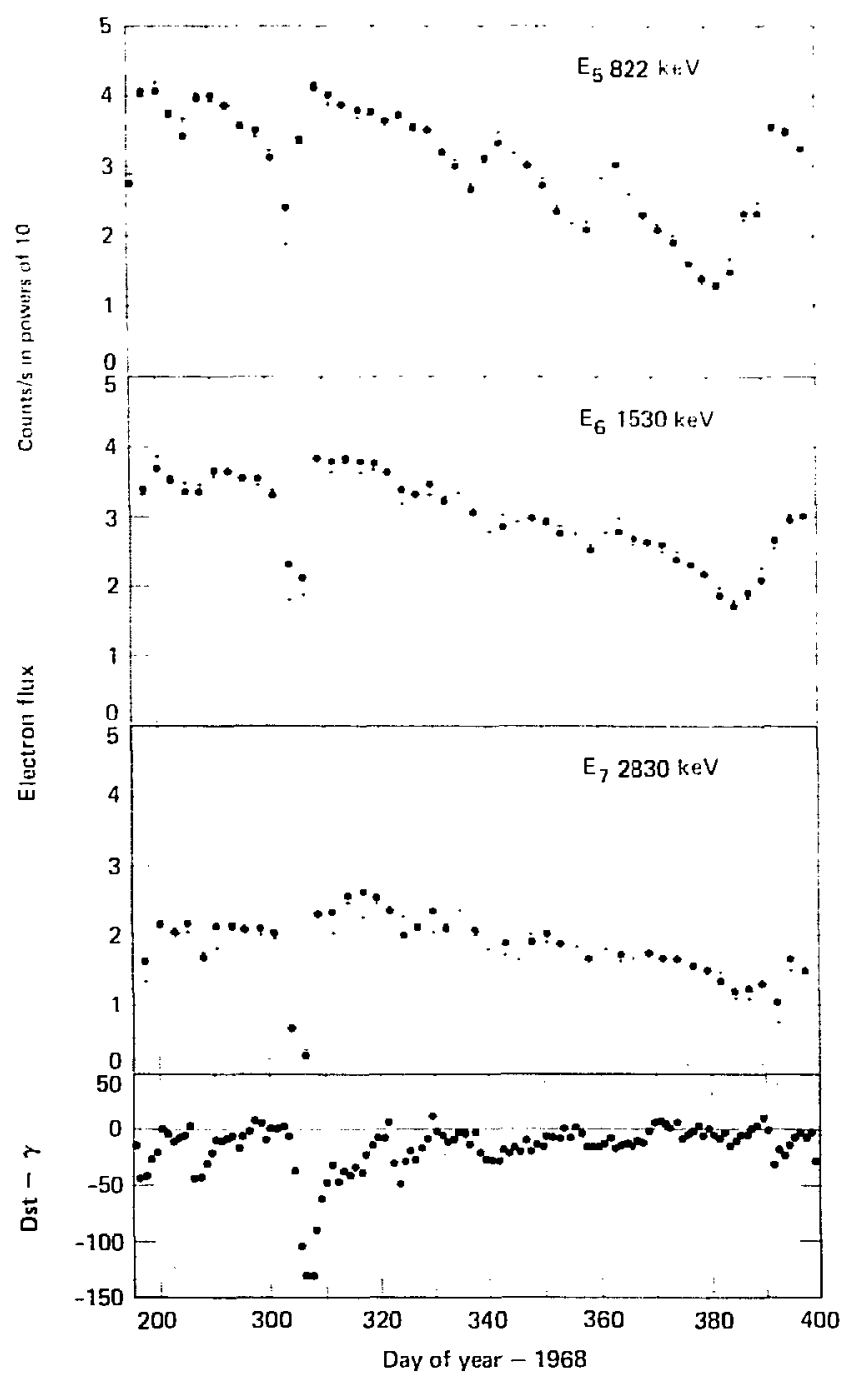

Fig. 15. Flux-vs-time plots for $L=4.00$ for Days $275-400,1968$. The data are corrected to 45.70 equatorial pitch angle. Note that the data are plotted as count rate minus background plus $1 \mathrm{c} / \mathrm{s}$. 


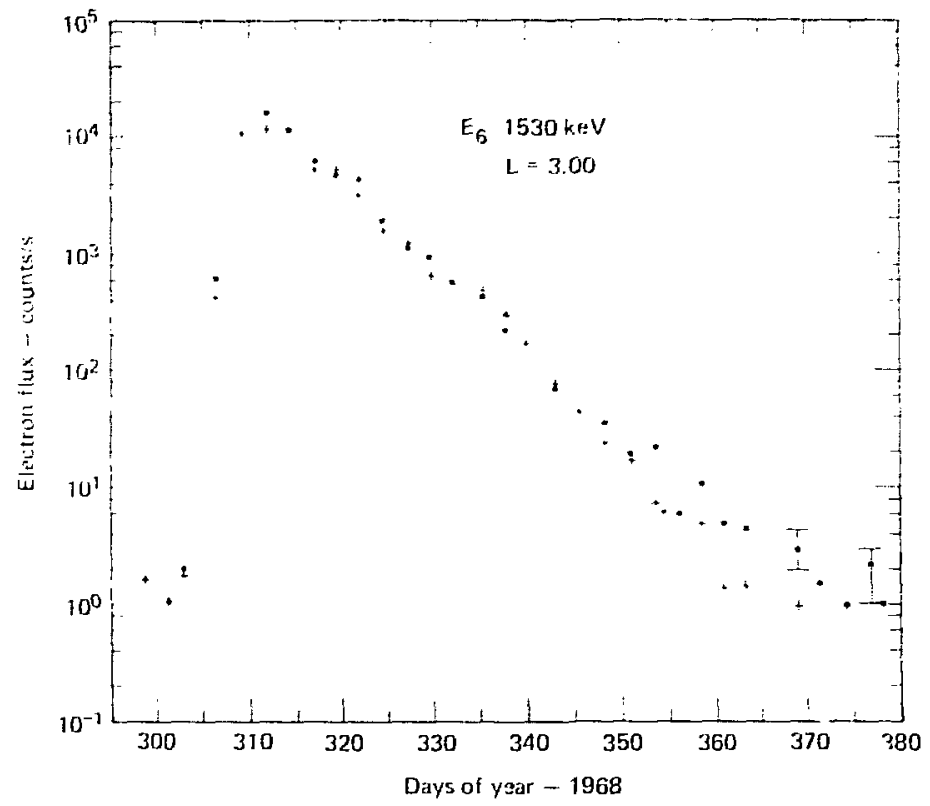

Fig. 16. Electron flux-vs-time at $L=3.00$ during Days $300-350,1968$, for $1530-\mathrm{keV}$ electrons. Note that the fluxes were at zero $\left(1 \times 10^{0}\right)$ just before injection on Lays 305-306. The data are corrected to 45:70 equatorial pitch angle.

TABLE 3. Measured mean decay in days.

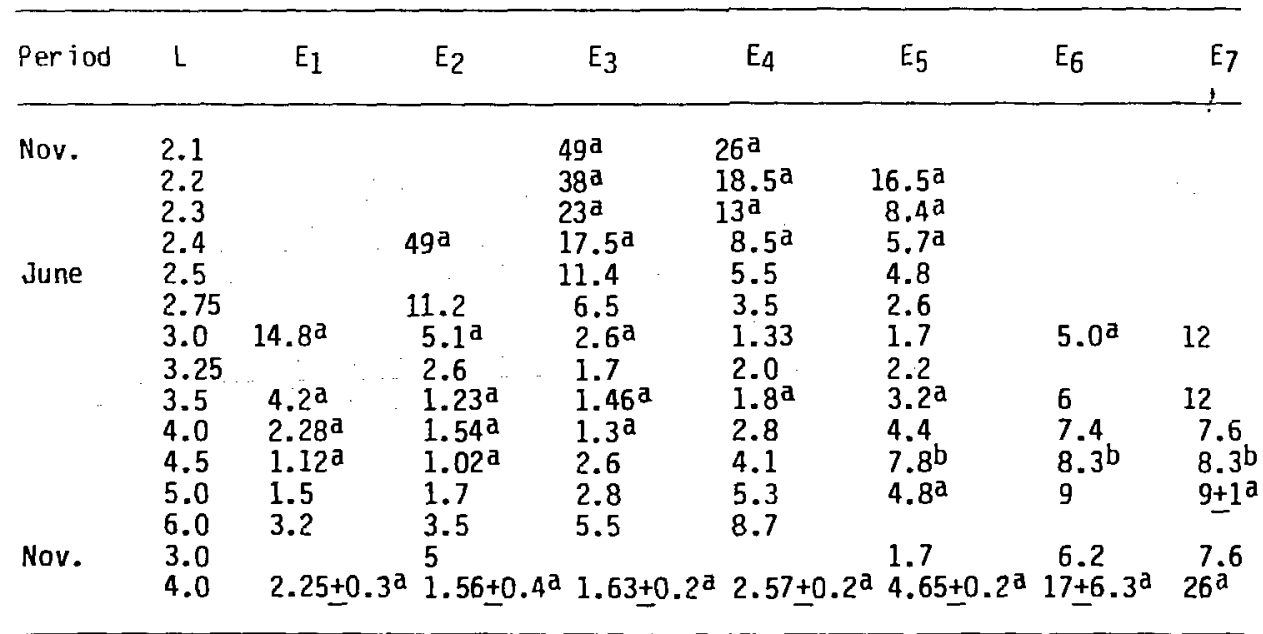




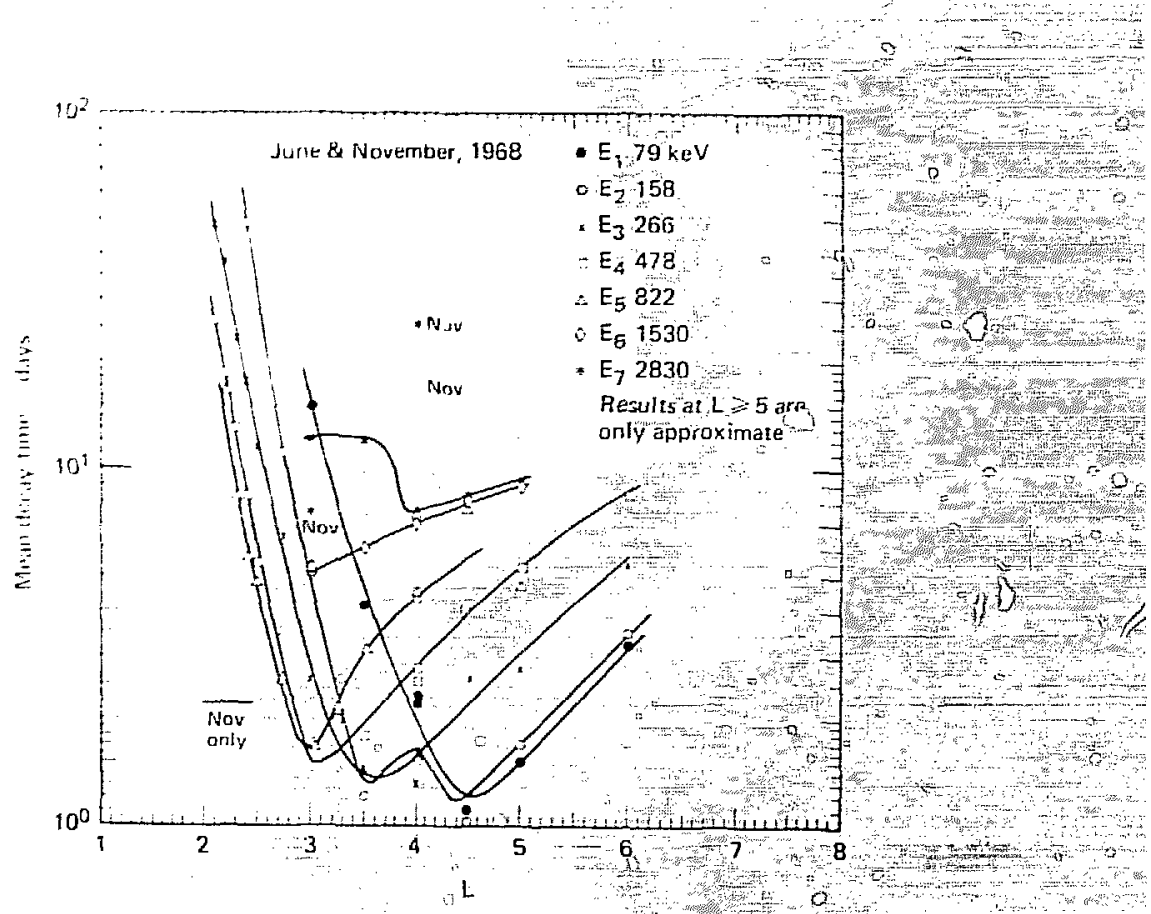

Fig. 17. Decays rates of $E_{1}-E_{7}$ electrons as detormined for the June and November 1968 periods. The $E_{1}-E_{5}$ data, we believe, are indicativenof. pitch-angle diffusion. Much of the $E_{6}$ data is probablyindicative of pitch-angle diffusion whereas only the lower three points for E 7 at $L=4$ to 5 could be due to pitch-angle diffusion.

remarkably consistent. Interestingly, though, the decay rates at pays 285 and 295 are greatly enhanced. The reason for th is has not been found.

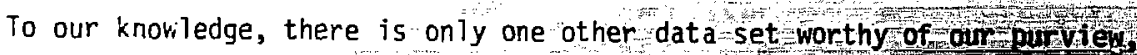

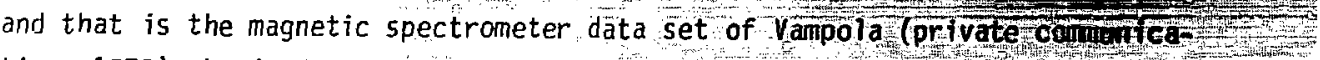

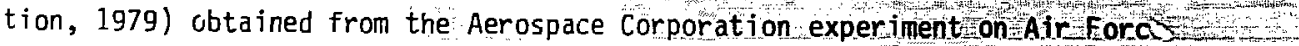
satellite 0y3-3. In this respect, it is a major scjentific disaster that recent NASA scientific satellites launched after $0 \mathrm{go} \mathbf{5}$ hecenot carfod instrumentation of adequate erier gy range and/or background rejection to obtain data complementary to the two present data sets. Vampola's data werefobtained at central energies of $300,475,712,957,1220,1490,1770,2010$, and $2310 \mathrm{keV}$ [Tomassian et a]., 1972] and are plotted in Figs. 18a-i. These data were acquired during 1966 and 1967 at values of $B$ varying from 0.05 to $0.01 \mathrm{G}$ and hence tend to be at high latitude. (Vampola corrected the data approximately to the equator by noting that $\log \mathrm{j}$ vs $\log B / B_{0}$ is often a fairly good straight line extrapolated to $B / B_{0}=1$. The procedure is inaccurate in the 

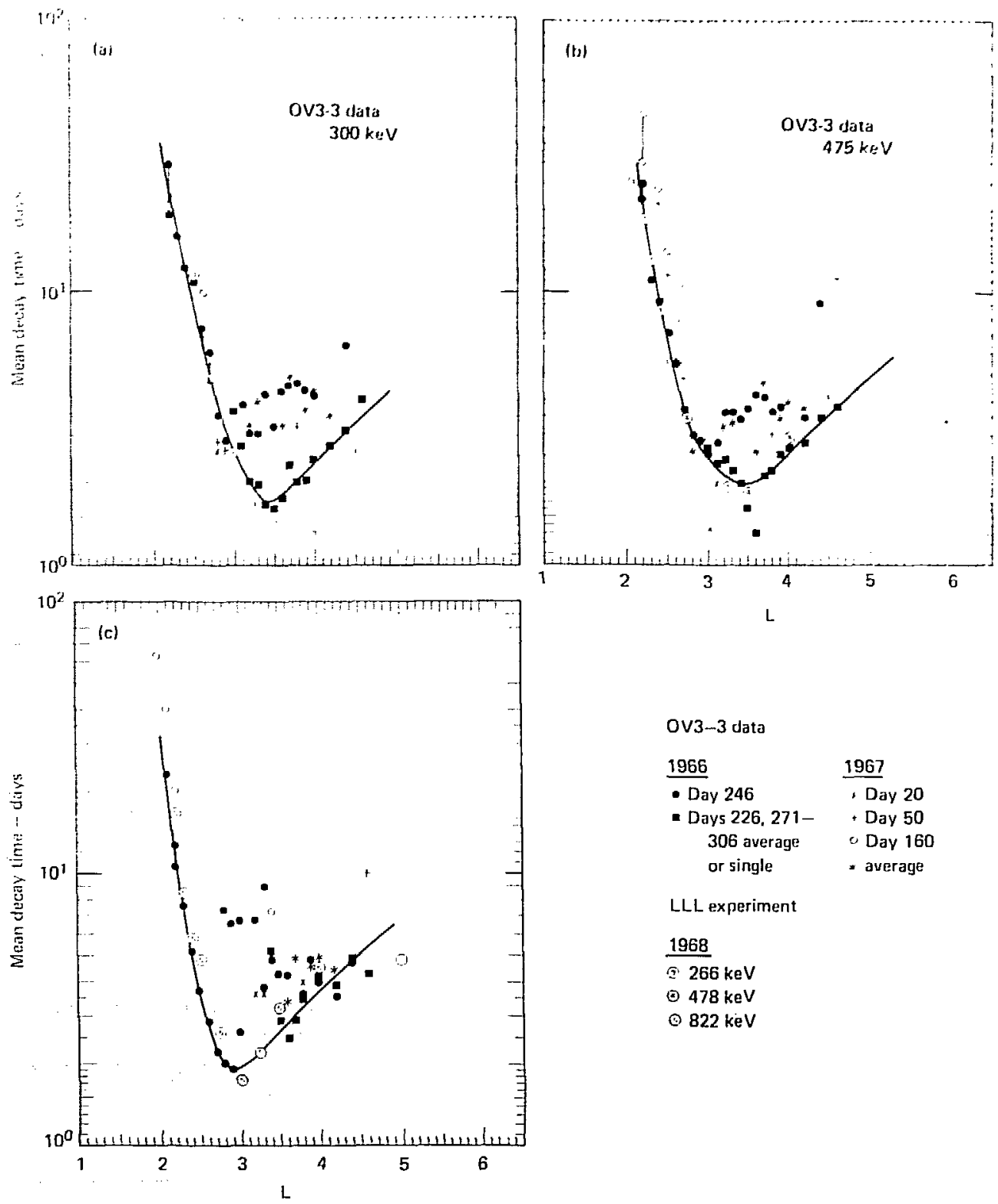

L

Ov3-3 data

1966

- Day 246

- Days 226, 271 306 average or single

LLL experiment

1968

(5) $266 \mathrm{keV}$

o $478 \mathrm{keV}$

(1) $822 \mathrm{keV}$

Fig. 18abc. 0V3-3 sateliite data for (a) $300 \mathrm{keV}$, (b) $475 \mathrm{keV}$, and (c) 712 $\mathrm{keV}$. The indicated traces are probably representative of pitch-angle diffusion; the higher values show effects of radial diffusion. The data were acquired courtesy of A. Vampola (private communication, 1979). 

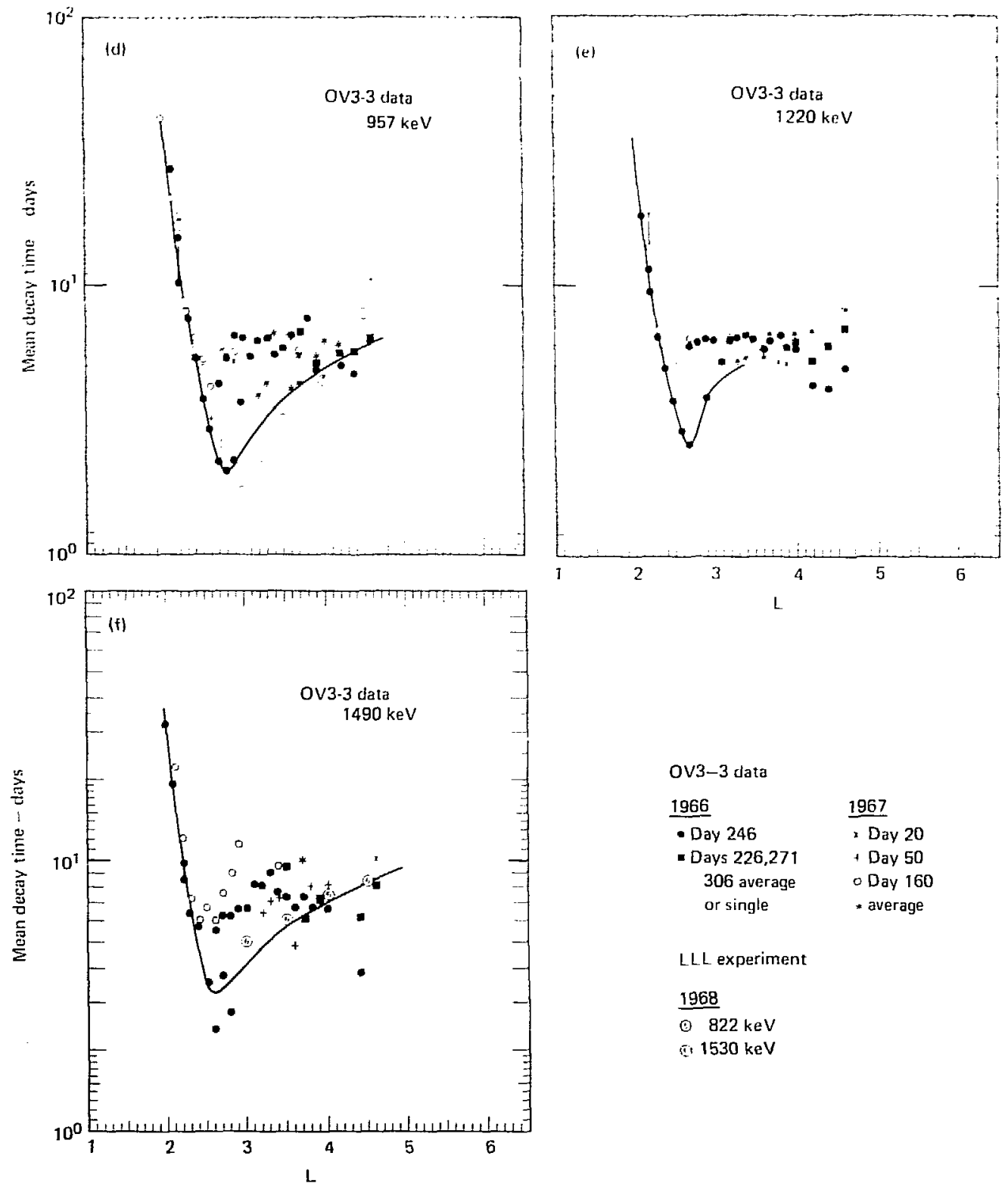

OV3-3 data

1966

- Day 246

- Days 226,271 306 average or single

LLL experiment

1968

(.) $822 \mathrm{keV}$

at $1530 \mathrm{keV}$

Fig. 18def. OV3-3 satellite data for (d) $957 \mathrm{keV}$, (e) $1220 \mathrm{keV}$, and (f) 1490 $\mathrm{keV}$. The indicated traces are probably representative of pitch-angle diffusion; the higher values show effects of radial diffusion. 


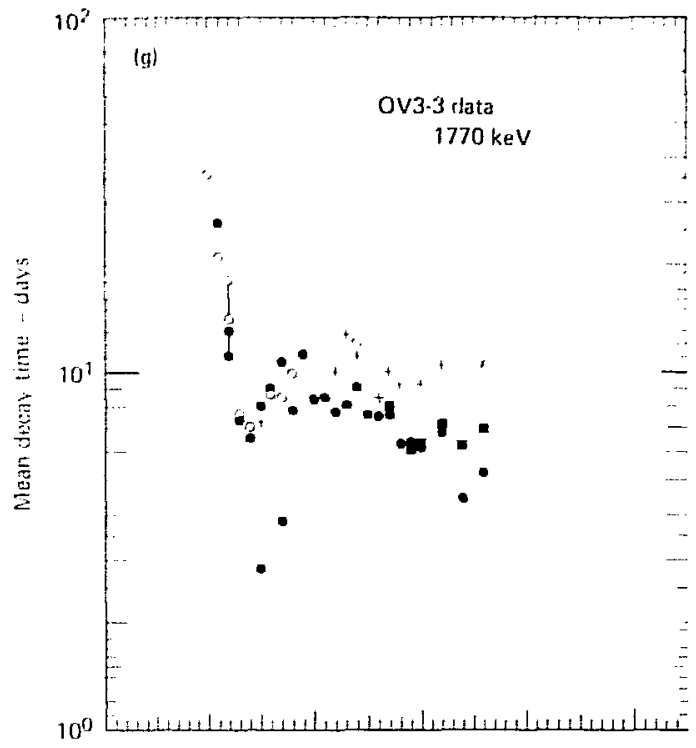

(h)
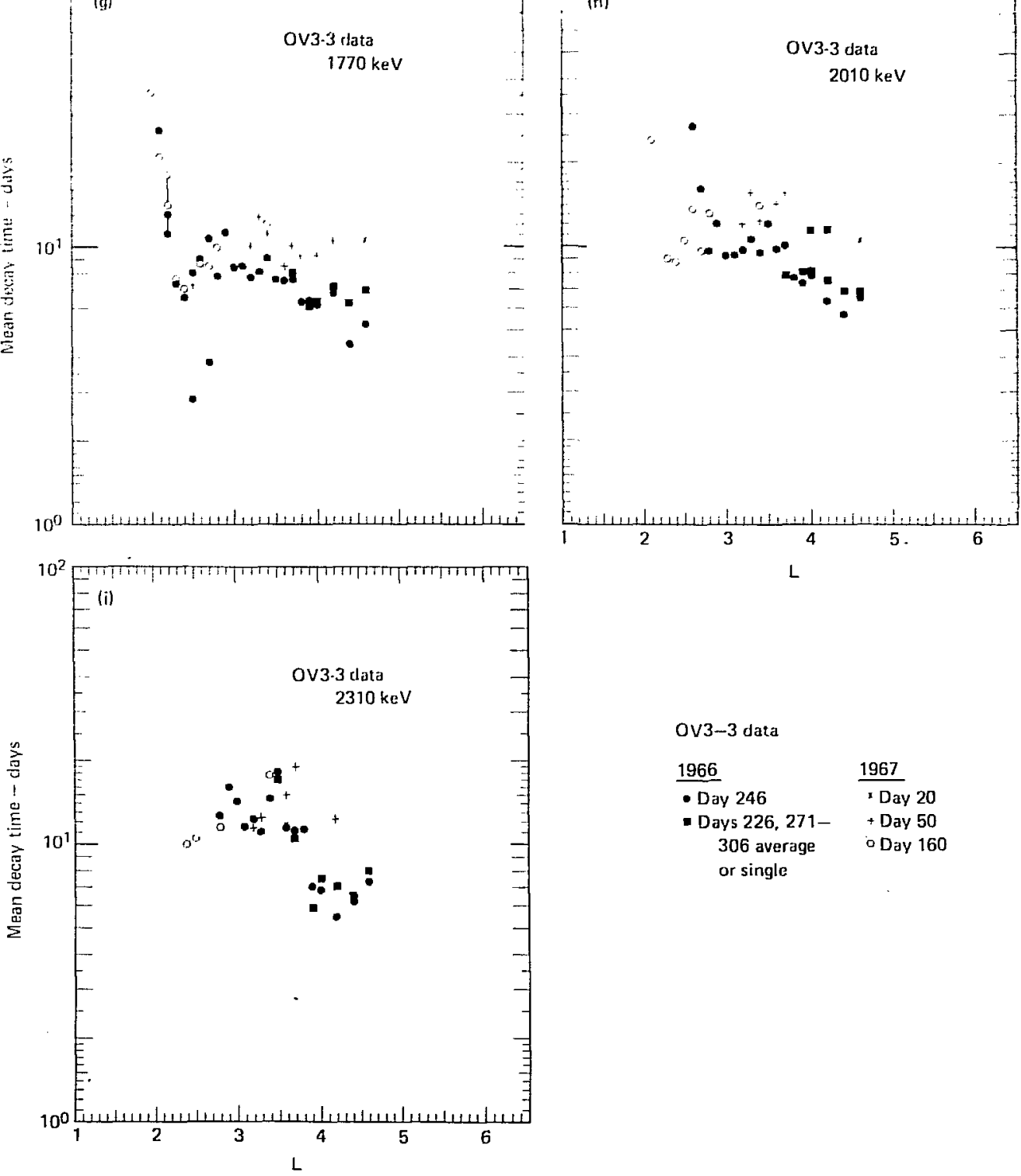

OV3-3 data

1966

- Day 246

- Days 226, 271306 average or single
1967

- Day 20

+ Day 50

- Day 160

Fig. 18ghi. 0V3-3 satellite data for (g) $1770 \mathrm{keV},(\mathrm{h}) 2010 \mathrm{keV}$, and (i) 2310 $\mathrm{keV}$. The indicated traces are probably representative of pitch-angle diffusion; the higher values show effects of radial diffusion. 
region of strong Landau effects in the PAD's, but it quite effectively corrects to a common base those fluxes with equatorial pitch angles less than about $45^{\circ}$.)

Major injections occurred on Day 246, 1966, and Day 160, 1967, with the 1966 injection providing the better data (see the APL data in Fig. 1 for perspective). As indicated on the figures, minor injections occurred at other times, and, as mentioned earlier, we searched for the shortest decay times and plotted all such data. The most reliable data, based on Day 246, 1966, are those at $L=2.2$ to -3.0 . At $L=2.7$, for some of the channels, two component decays were found, and the two components are plotted in the figures. For the 1220- and 1490-keV channels, the minimum decay times were hard to determine and are not too accurate. To provide a much needed perspective, we also have included data at approximately the same energy from our experiment. As a result of our considerations, we feel justified in sketching in the bottom envelope of the data to the Vampola data and presenting the results as pitch-angle diffusion. For the $1770-\mathrm{keV}$ channel and higher, it is difficult to identify anything we might want to call pitch-angle diffusion.

\section{ELECTRON SPECTRA}

The evolution of the electron spectra following the storm-time injections is especially interesting. We first presented a brief study of these results [West et al., 1970], and Vampola et al. [1977] has presented somewhat similar results; otherwise, the evolution of the spectral features as presented here is both new and unique.

Figures 19 ab show the evolution of spectra at $L=3,3.5,4.0$, and 4.5 following the Day-163 injection. (Note that data points for both inbound and outbound portions of the orbit are usually shown along with the respective $\lambda_{I}$. The log-average spectra are shown.) A pre-injection spectrum is shown by the Day-158 data; note for $L=3.0$ and 3.5, there are no measureable electron fluxes above - $300 \mathrm{keV}$. The electron spectra for Day 163 possibly do not show the full injection since marked increases are apparent on Day 168, especially at > $300 \mathrm{keV}$. The spectra for Day 168-184 show the general evolution of the spectral features in the data. (A small injection occurred before the next available data on Day 187.) The data at $L=3.5$ and 4.0 show both the growth and decay of the higher-energy electrons and the marked decay 

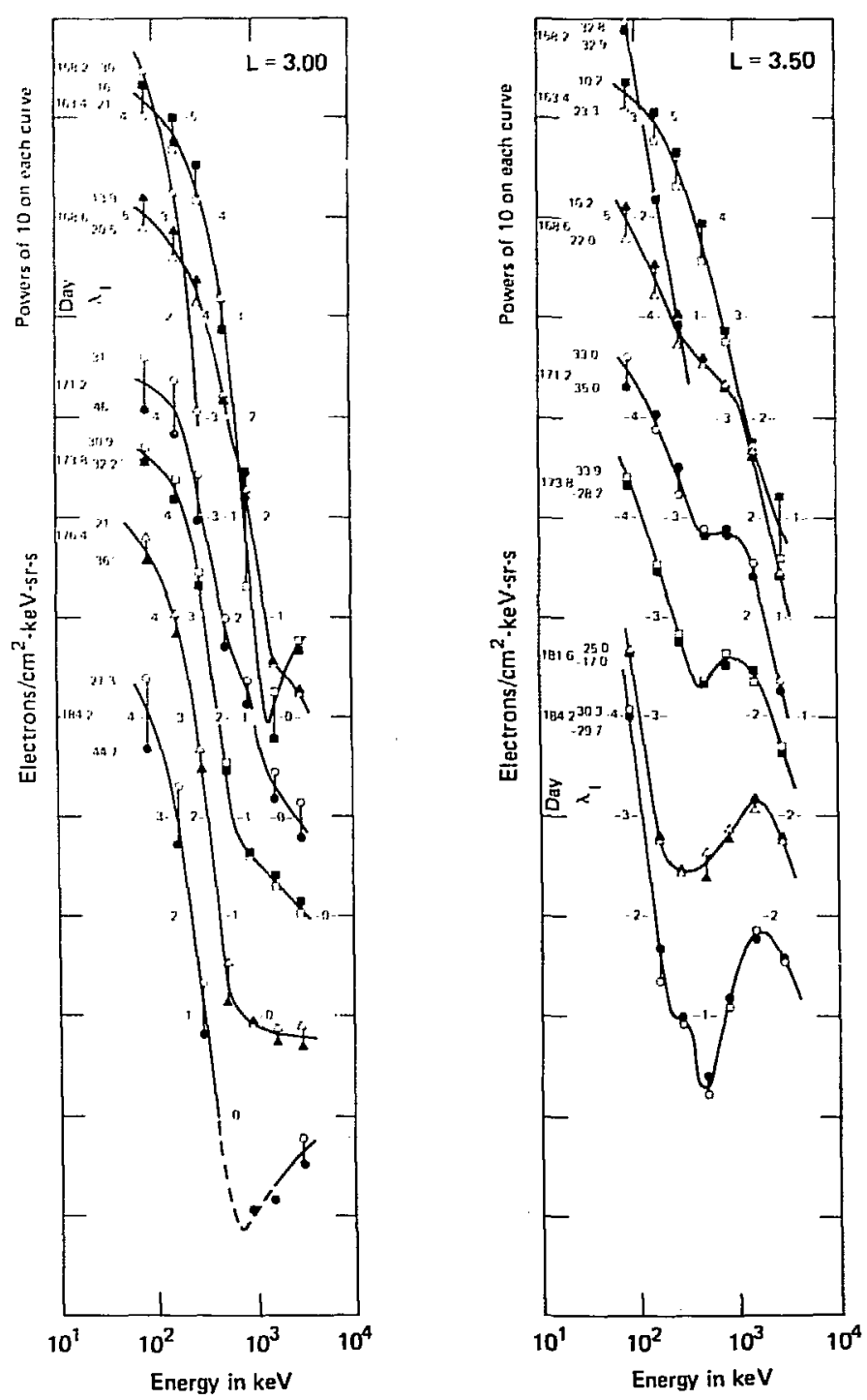

Fig. 19a. Electron spectra before, during, and after the magnetic storm of Day 163, 1968. A new injection that occurred shortly after Day 184 makes further study not too meaningful. 

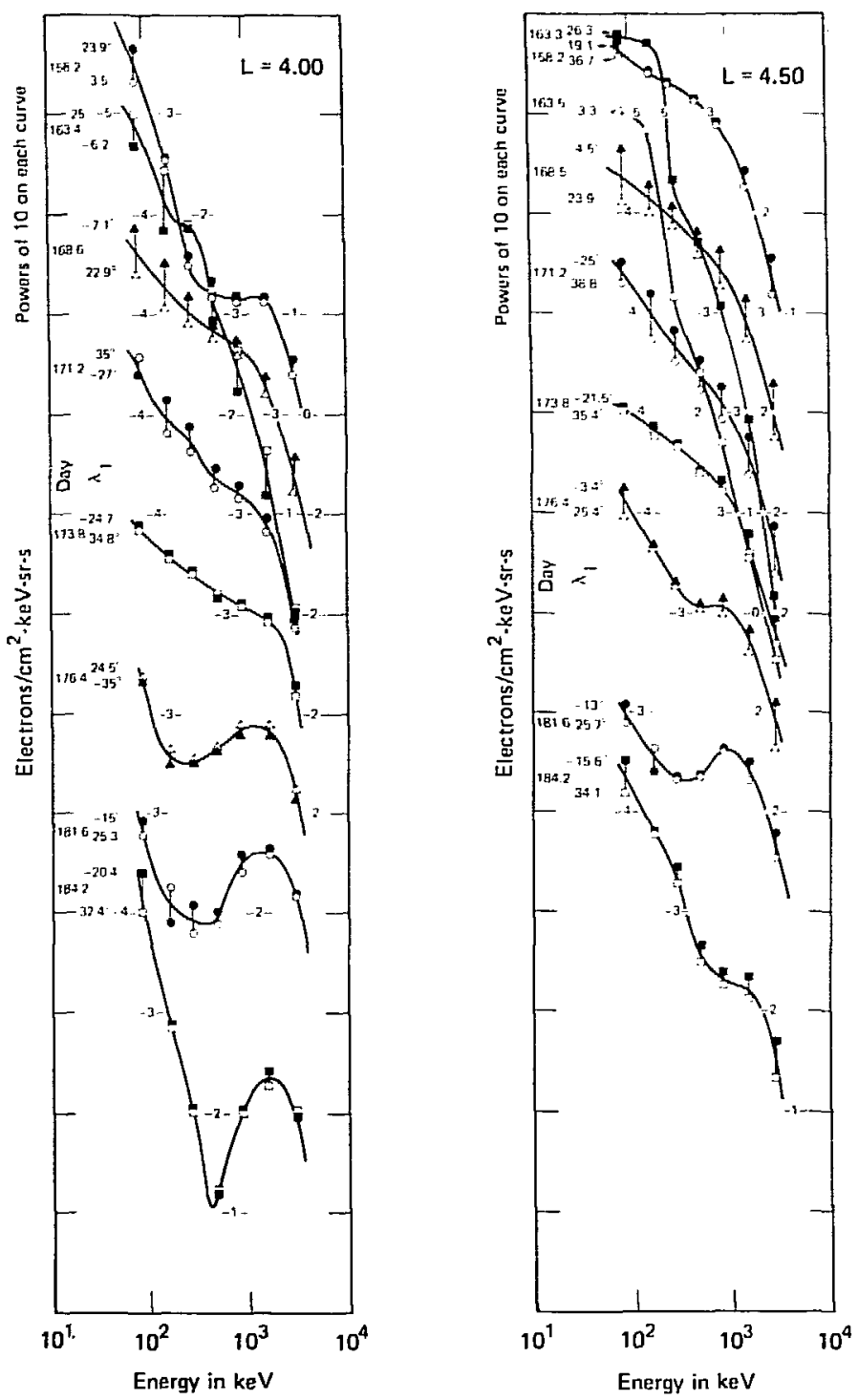

Fig. 19b. Continuation of the electron-spectra study (begun in Fig. 19a) associated with the Day-163 storm. 
of electrons - 200 to $500 \mathrm{keV}$; note that the plots tend to emphasize the decay of those electrons that have their most rapid decay at $L=3.50$ and 4.0 .

Figure 20 shows spectra obtained at $L=3.0$ and 4.0 following the Days 305-306 storm injection. By comparing the $L=3.0$ data to those of the June period, we note that there was injection to much lower L-shells during Days 305-306. For the latter period, we were fortunate to have almost a month fiee of the injections that would have greatly affected the spectra; although something happened - Day 320, the evolution of the spectra was not affected greatly. The significant aspect in the comparison of the two data sets is that, despite the wide range in magnetic activity for the two periods, the features in the resulting spectra are much the same.

We find it very interesting and significant that the spectrum of electrons that exists in these regions some weeks after a magnetic storm (as a result of the diffusive rearrangement of electrons) tends to be rather hard. This observation is especially significant in the evaluation of data from interral electon spectrometers and, of course, is significant to the very practical problem of damage to satellite electronics.

In deference to those interested in radiation damage, we present Fig. 21. We noted that the fluxes at $L=6.0$ in the study of electrons for Days 147-220, 1968, did not show very marked fluctuations, even during the Day-163 storm injection (a relatively mild storm). Hence we performed a log-average of the counting rates along with the probable deviation of $\log j_{1}$. The data were not corrected to the equator but used at their $\lambda_{I}$ of observation, which averaged $22^{\circ}$. Since these electrons are so close to the injection region assimed to be that primarily associated with substorms, we do not expect the spectrum at $L=6.0$, as we have presented it, to be very dependent on whether we are at solar minimum or maximum.

Vampola (private communication, 1979) has brought to our attention that his published average-spectrum of OV1-19 data acquired in 1969 [Vampola et a1., 1977] at $L=6.0$ agrees extraordinarily wel1 (10w by 20-40\%) with our spectrum in Fig. 21. There is a qualification, however, in that Vampola's data were taken at $B \simeq 0.05 \mathrm{G}$, which translates to $\lambda_{I}=52.8^{\circ}$ or $\alpha_{0}=9.75^{\circ}$. We est imated that the needed pitch-angle correction for the comparison is - X4, which places the Vampola data near the top of our probability bars. Even with this qualification, we consider the results of the comparison good. 

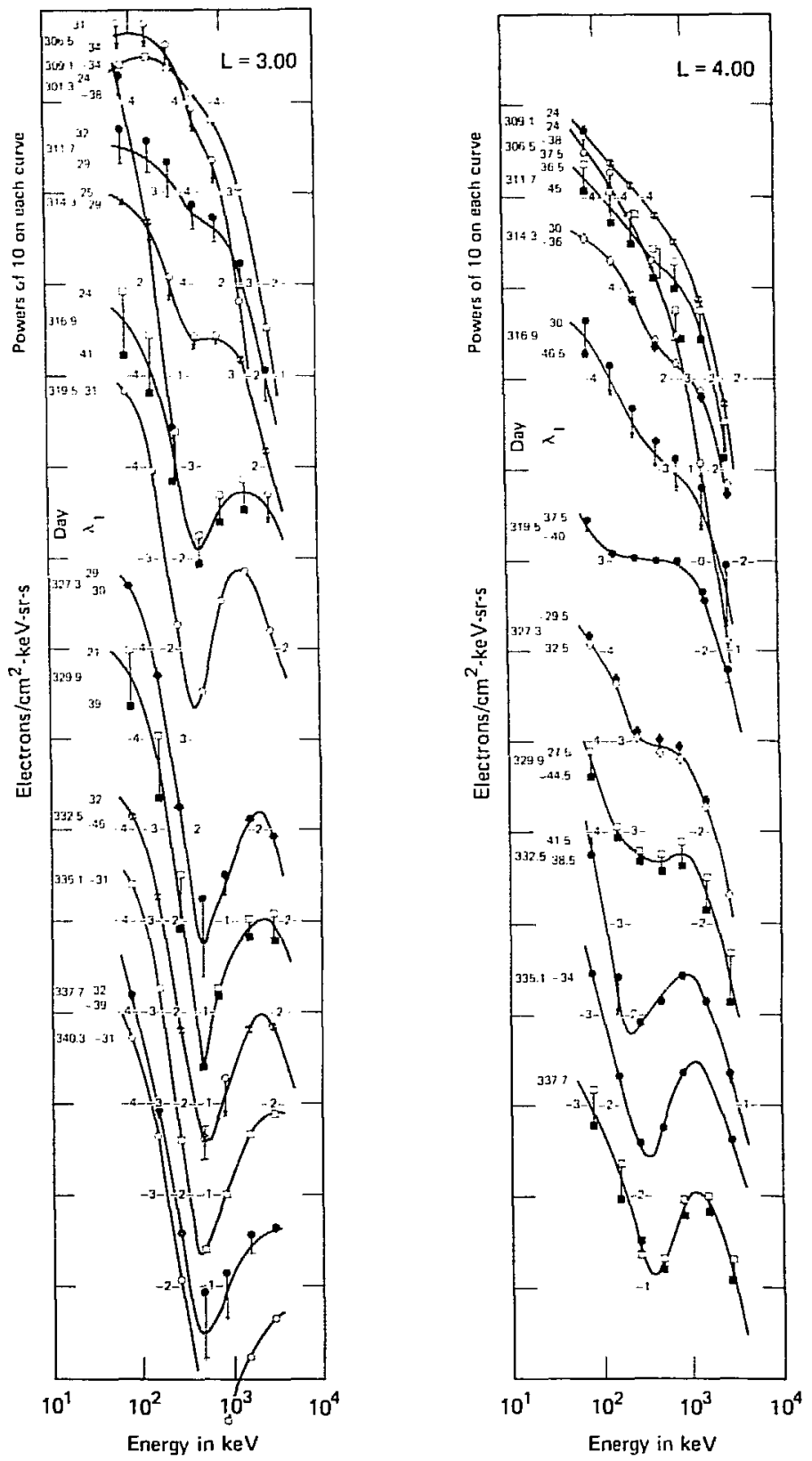

Fig. 20. Study of evolution of electron spectra following the main-phase injections on Days 305-306, 1968. From Fig. 15, we note that an additional injection, occurring $\sim$ Day 320 , shows in the $E_{1}-E_{4}$ data but not in the $E_{5}-E_{7}$ data. 


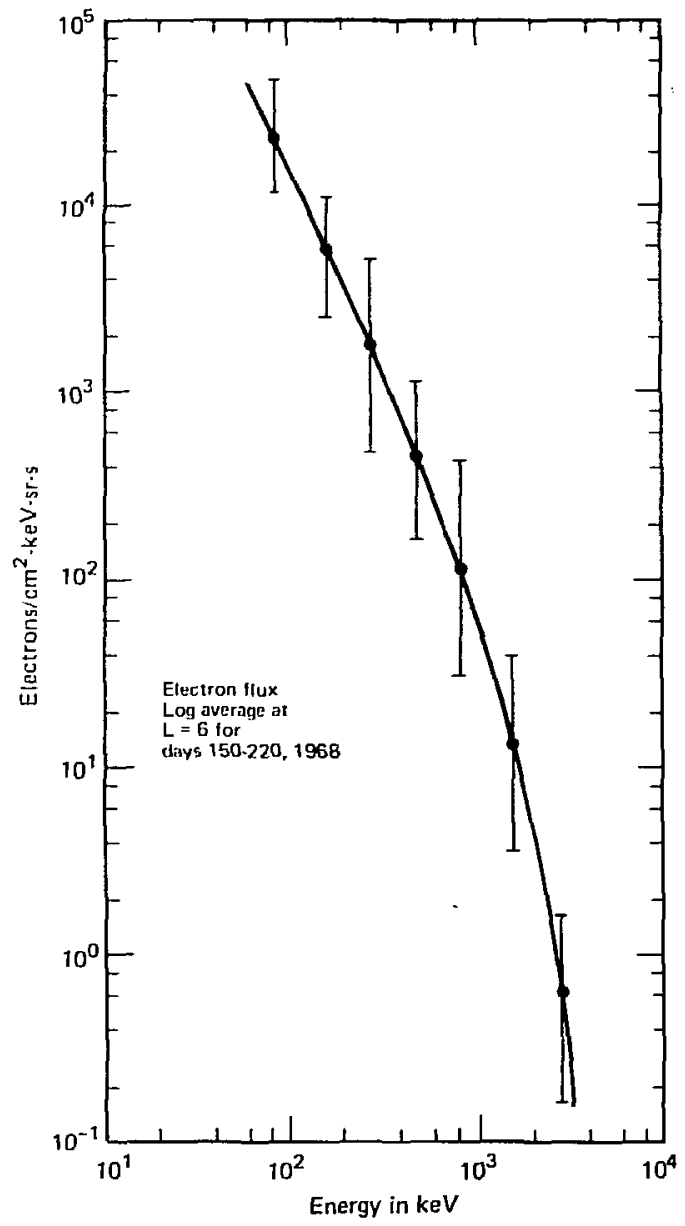

Fig. 21. Average electron flux for Days 150-220, 1968. The error bars show the protichle value of $\log j_{1}$. The data are not pitch-angle corrected but are representative of the flux to be expected at $\lambda_{I}=40$ to $0^{\circ}$ during all but very fi=turbed periods.

\section{DIFFUSION ANALYSIS}

To show that the minimum lifetimes observed in our studies can be reasonably identified with pitch-angle diffusion, we performed a radial-diffusion analys is of the November data. Radial diffusion is 
customarily treated under the assumption that the first and second adiabatic invariants are conserved. The pitch-angle diffusion is assumed to result from wave-particle interactions that violate only the first invariant. During radial diffusion (or convection), the mirror field changes as

$$
B_{m} \propto L^{-n}
$$

where $n$ is approximately a constant equal to 3 at the equator and decreases to 2 in the limit of zero-pitch angle $\left(B_{m}+\infty\right)$. We define a dimensionless variable:

$$
n=\frac{p^{2}}{m^{2} c^{2}} L^{n}=\frac{E\left(E+2 m c^{2}\right)}{m^{2} c^{4}} L^{n} \text {, }
$$

which is proportional to the magnetic moment when $n=3$; at such a time, it is equivalent to MeV/G when divided by 1.217 . This variable is approximately conserved in both radial and pitch-angle diffusion. A diffusion equation written in the three variables $\alpha_{0}, L$, and $n$ is therefore reduced to an equation with only two differential operators and no cross terms [Wa]t, 1970; Schulz and Lanzerotti, 1974]:

$$
\begin{aligned}
\frac{\partial f}{\partial \mathrm{t}}= & L^{-2+3 n / 2} \frac{\partial}{\partial L}\left[\bar{D}_{L L} L^{2-3 n / 2} \frac{\partial f}{\partial L}\right] \\
& +\frac{1}{\cos \alpha_{0} \sin \alpha_{0} \tau_{b}} \frac{\partial}{\partial \alpha_{0}}\left[\bar{D}_{\alpha_{0} \alpha_{0}} \cos \alpha_{0} \sin \alpha_{0} \tau_{b} \frac{\partial f}{\partial \alpha_{0}}\right] \\
& + \text { (Sources) }- \text { (Atmospheric Loss) }
\end{aligned}
$$

where $f \bar{F} f\left(\alpha_{0}\right)$ is the phase space density and $\tau_{b}$ is the bounce period. The diffusion coefficients, $\bar{D}_{\alpha_{0} a_{0}}$ and $\bar{D}_{L L}$, are averaged over the bounce period [Lyons, Thorne, and Kennel, 1972].

For equatorially mirroring particles, the $L$ diffusion operator becomes

$$
L^{5 / 2} \frac{\partial}{\partial L}\left[\bar{D}_{L L} L^{-5 / 2} \frac{\partial}{\partial}[] .\right.
$$


The phase-space density for this case is

$$
f_{0} \times j_{\perp 0} /\left(p^{2} / m^{2} c^{2}\right)=j_{10} L^{3} / \eta \text {. }
$$

For particles mirroring well off the equator, $n$ does not change much until the mirror latitude exceeds $-70^{\circ}$. An interesting case is $\alpha_{0}=40^{\circ}$, for which $n=8 / 3$, and the diffusion operator is

$$
L^{2} \frac{\partial}{\partial L}\left[\bar{D}_{L L} L^{-2} \frac{\partial}{\partial L}\right]
$$

The power of $L$ is (coincidentally) the same as that used in much of the early literature on radial diffusion.

The solution of the diffusion equation to find $\bar{\sigma}_{L L}$ is greatly simplified if $\bar{G}_{a_{0} \alpha_{0}}$ can be evaluated separately, either by theoretical analysis [e.g., Lyons, Thurne, and Kennel, 1972] or by analysis of cases in which radial diffusion can be ignored. The latter alternative might seem impossible to implement, but there may be situations in which the effects of radial diffusion are weak. This can occur when the pitch-angle distribution is far from its lowest normal mode [Wa]t, 1970]. It is difficult to take advantage of this situation because the distribution may be changing very rapidly, thus hindering the evaluation of decay rates. It is often easier to look for instances where the pitch-angle distribution decays exponentially while maintaining its shape. This ensures that the distribution has reached its lowest normal mode. If electrons over a broad $L$ range decay exponentially but with different rates, it may be assumed that pitch-angle diffusion dominates. (One caution: the radial diffusion rate may fortuitously be of just the right magnitude over the $L$ range that the above situation prevails; the distribution then on ly appears to be in its lowest mode.)

We believe that the decay data observed during the June storm and, in genera1, the rapid decays as observed for the 0V3-3 data are fairly good measurements of the pitch-angle diffusion rates, and we have used them to analyze the data observed during the November storm in terms of radial diffusion. Most assuredly for the higher energy groups for the June storm, there are radial diffusion results (note the growth in the fluxes for some of the cases), but the rases (at least most of them) are so drastically different 
between the two periods that we feel no serious errors are being made. The pitch-angle diffusion decay rates used for the present study are shown in fig. 22 and were derived, of course, from Figs. 17 and 18. Obviously, these decay rates include the effects of atmospheric loss.

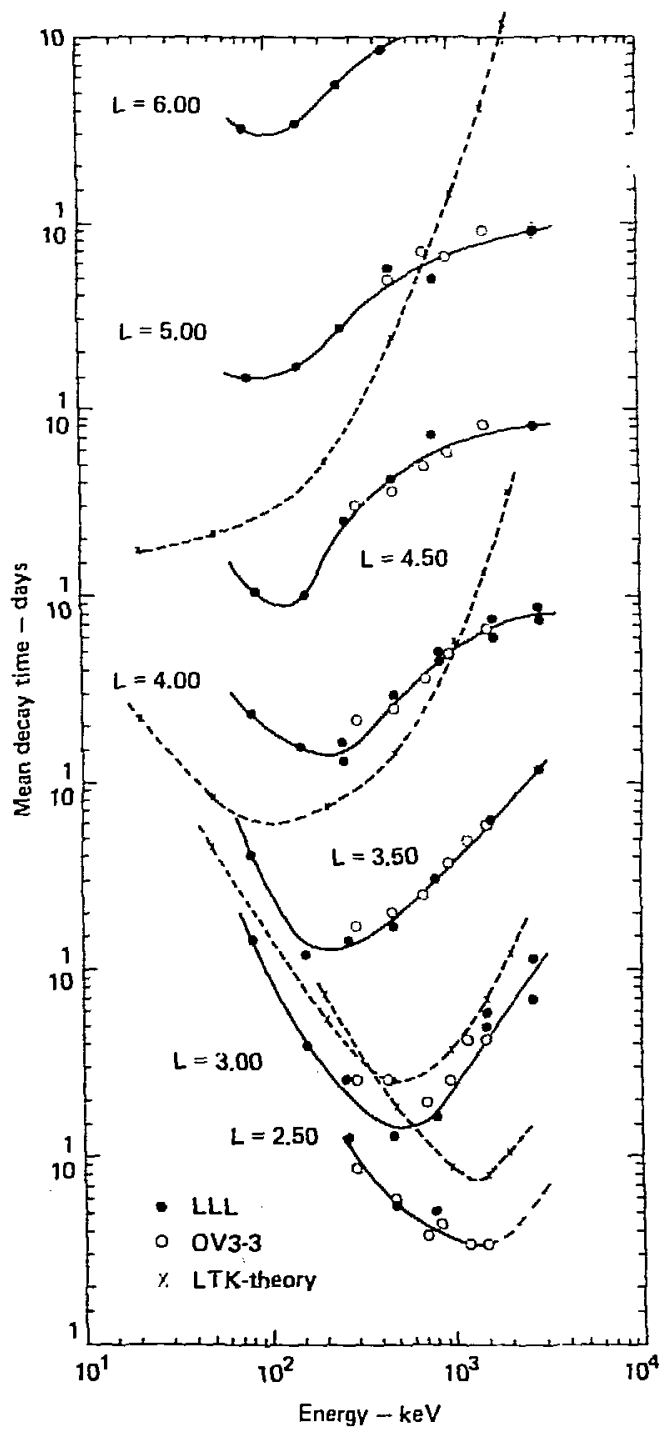

Fig. 22. Pitch-angle diffusion lifetime -" constant L. The results were derived irom Figs. 17 and 18 . The dashes curve is from the theory of Lyons, et a1. [1972]. 
Our diffusion equation, as presented above, includes the sources. For the period that we selected (after the main-phase injection), we believe that all local sources have beer, turned off, and we assume any continuing source is located at or beyond the L-limit of our analysis (i.e., at $L>7$ ). Finally, we wish to solve

$$
\frac{\partial f_{0}}{\partial t}=L^{-5 / 2} \frac{\partial}{\partial L}\left[L^{5 / 2} \bar{D}_{L L} \frac{\partial f_{0}}{\partial L}\right]_{\eta}-\frac{{ }^{f_{0}}}{{ }^{T_{P A D}}},
$$

in which the diffusion is at constant $\eta$ and ${ }^{T} P A D$ is the pitch-angle diffusion decay rate.

An obvious approach to the solution (see Schulz and Lanzerotti [1974] for a discussion of various approaches to the solution of the diffusion equation) is to assume that $\bar{u}_{L L}$ varies as a power law of $L$, most typically $\bar{D}_{L L}=k_{1} 10$, and solve immediately for $k$, where $\partial f_{0} / \partial t$ and $f_{0} / \tau_{\text {pAD }}$ are known. The method relies upon determining $\partial^{2} f_{0} / \partial L^{2}$, which can be uncertain despite the data smoothing employed in such an analysis. We have chosen an approach that minimizes the uncertainties in the data; the method is based upon the first integral of the diffusion equation:

$$
\bar{D}_{L L}(2)=\frac{\int_{1}^{2} L^{5 / 2}\left(\frac{f_{0}}{\tau_{P A D}}+\frac{\partial f_{0}}{\partial t}\right) d L+L_{1}^{5 / 2} \bar{D}_{L L}(1) \frac{\partial f_{0}}{\partial L_{1}}}{L_{2}^{5 / 2} \frac{\partial f_{0}}{\partial L_{2}}} .
$$

One can start at a high value of $L$, where $\partial f / \partial L=0$, and generate $\bar{D}_{L L}(2)$, or, preferably, start at a low value, estimate a diffusion coefficient at the lower boundary (which can be corrected later by extrapolating from the nigher calculated values), and then integrate for increasing $L$. We have used the latter approach.

Figures 23 and 24 show the equatorial distribution functions for two periods (Days 309.1-322.1 and 324.7-335.1) following the main-phase injection on Day 306 . These plots were generated by first transforming the data to the equator, making log-log spectral plots of the data, and interpolating by hand 


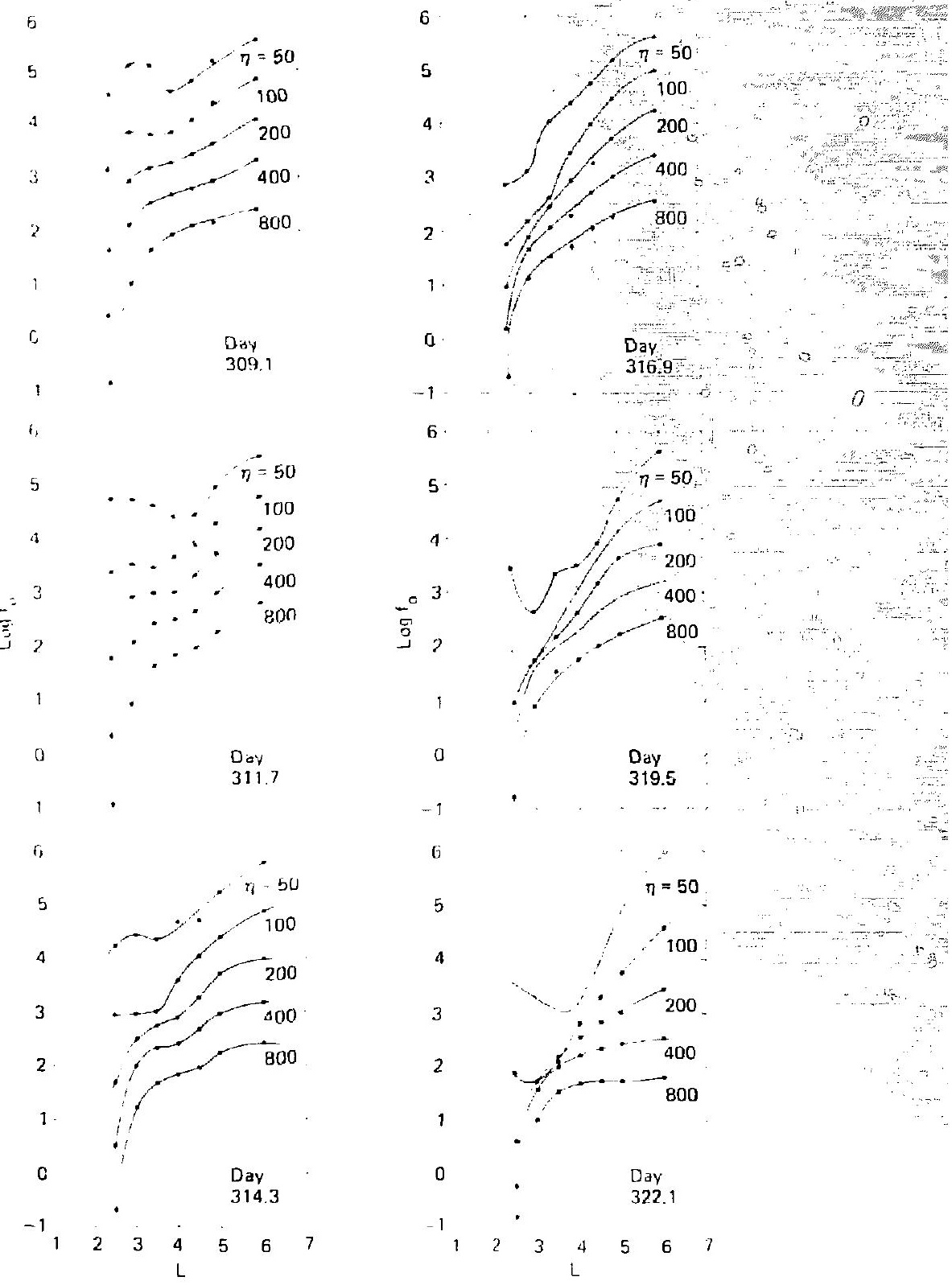

Fig. 23. Equatorial distribution functions for Days 339.3-322.1. Vote that for $L$ changing from $6-2.5$ at $\Pi=50$, E varies from $56-536 \mathrm{ieV}$; For $\eta=100$, $107-879 \mathrm{keV}$; for $\pi=200,198-1387 \mathrm{keV}$; for $\pi=400,352-2124 \mathrm{keV}$; and for $n=$ $800,597-3180 \mathrm{keV}$. 


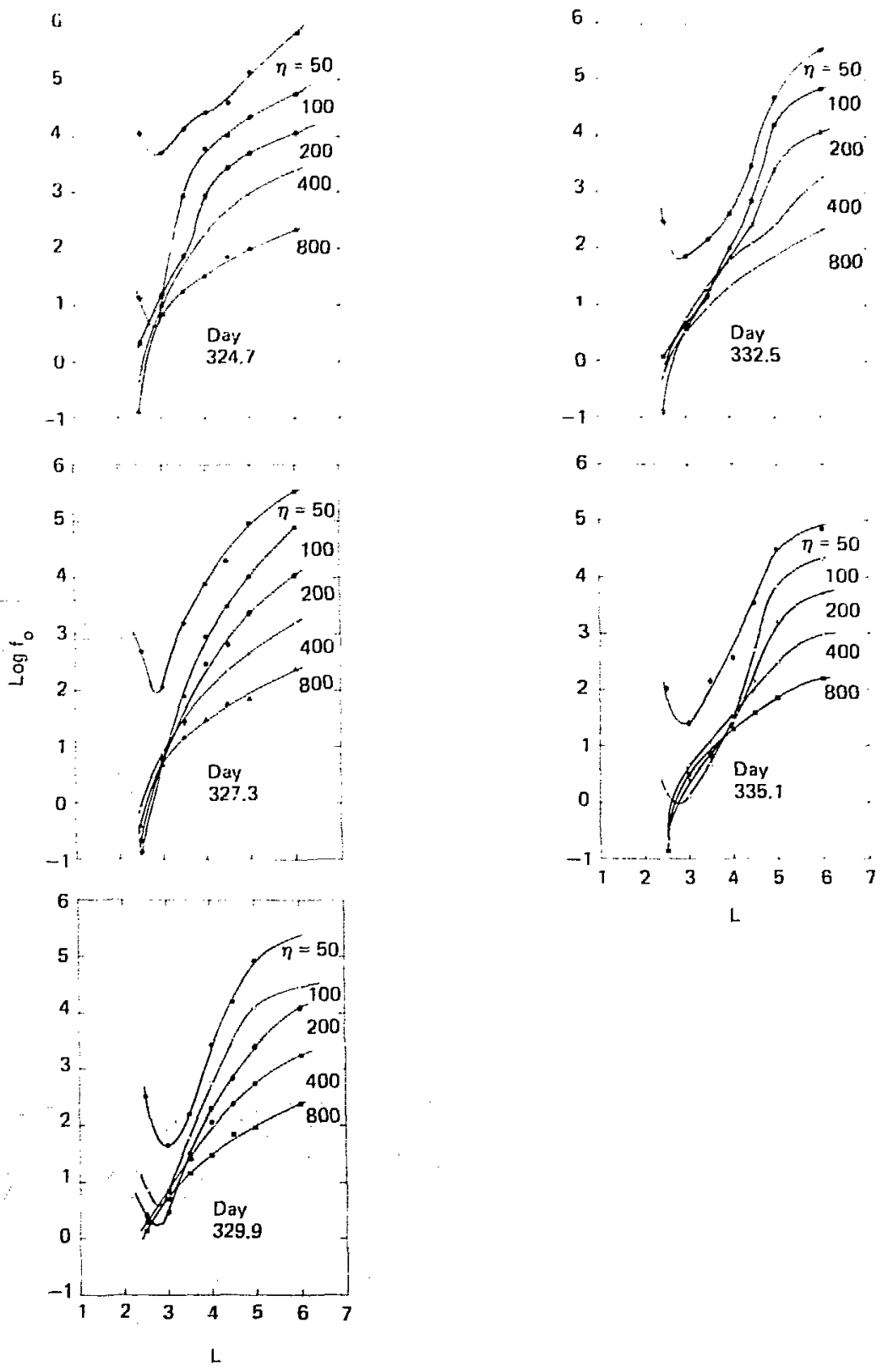

Fig. 24. Equatorial-distribution functions for Days 324.7 to 335.1 . 
between data points to obtain values of $f_{0}$ at the required energies. Figure 25 shows plots of $f_{0}$ at constant $n$ as a function of time. There is a change in the trend of the data about Day 322, necessitating treating the two periods separately. For each value of $\eta$, the data were fitted to $e^{-t / \tau}$ by least squares; the data were not always perfectly represented by an exponential expression, but such treatment represents the best compromise. The averaged values of $f_{0}$ were plotted at the center of the respective time intervals, as shown in Fig. 26. For subsequent calculations, the data in Fig. 26 were fitted by least squares to $\ln \mathrm{f}_{0}=\mathrm{a}+\mathrm{bL}^{-1}+\mathrm{CL}^{-2}+\mathrm{dL}^{-3}+\mathrm{eL}^{-4}$ and
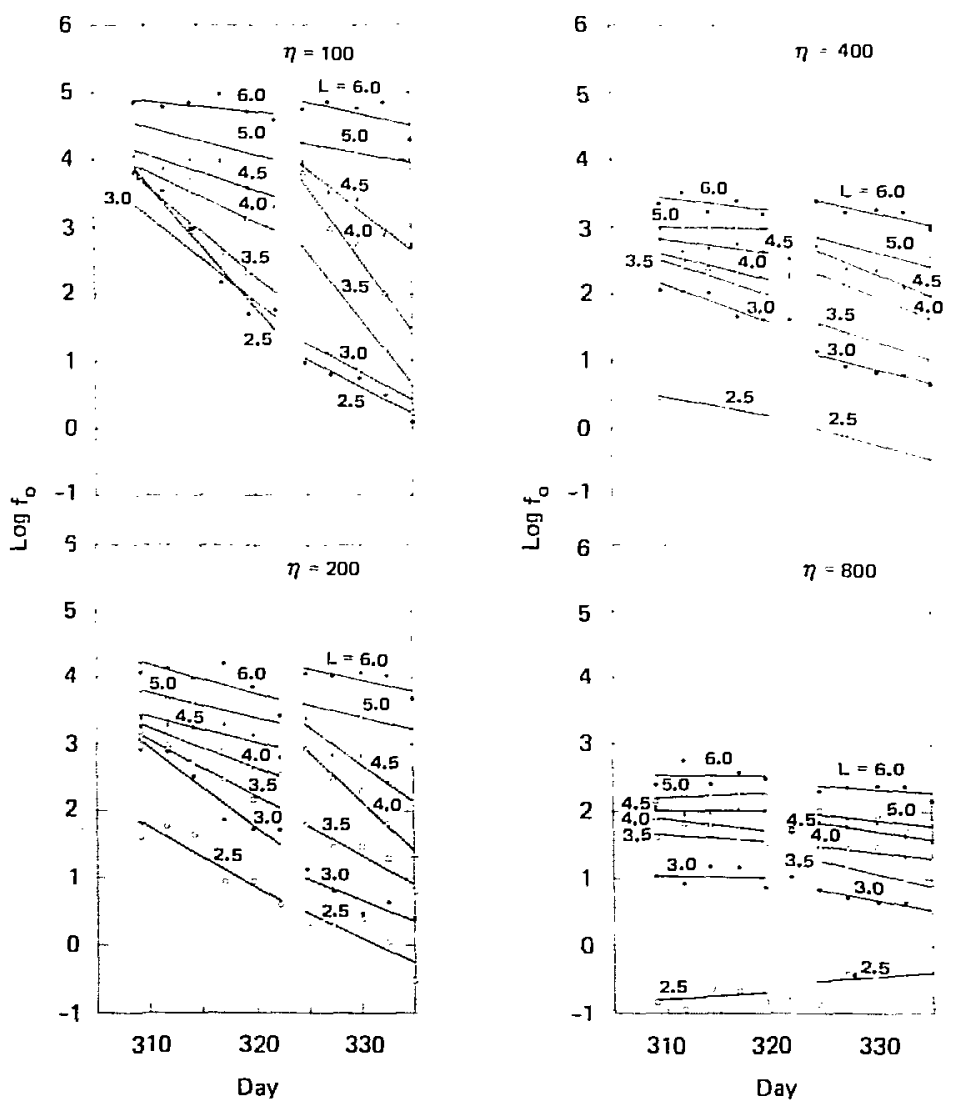

Fig. 25. Distribution function $f_{0}$ at constant $\eta$ as function of time for Days 309.1 to 335.1 . The straight lines are least-squares fits to the data for the indicated time periods. 

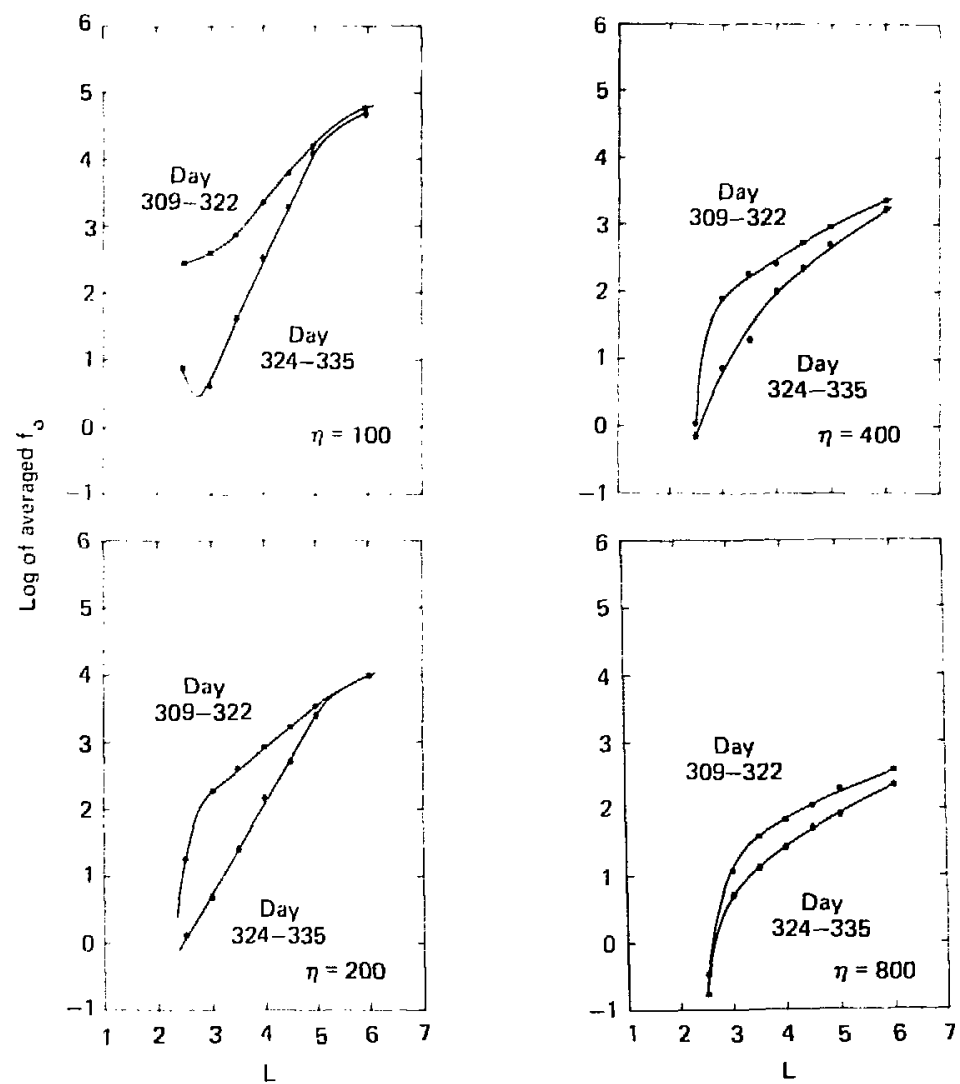

Fig. 26. Equatorial-distribution functions representing the least-squares average at the center of the indicated intervals based on the distribution functions of Fig. 25.

the fitted results provided the $\partial f_{0} / \partial L$ values used in the $D$-determination. The results of the diffusion analysis are shown in Figs. 27 and 28.

Figure 27 shows the results of the diffusion calculation using the two approaches. The solid dots show the results of the full calculation, and the open dots show the results with $\partial f_{0} / \partial t=0$. The latter approach indicates the maxinum errors possible in case of errors in the lifetime determinations. Figure 28 shows $\bar{\sigma}_{L L}$ for the two analyzed periods in a more conventional log-log plot. In Figs. 27 and 28, we first draw attention to the $\eta=200$ curve for Day 324-335 at $L=4-4.5$. The energy range is 527-402 keV (energies at which decay is moderately fast), and it is evident that the apparent decay at this time is close to that we assigned to pitch-angle diffusion. 

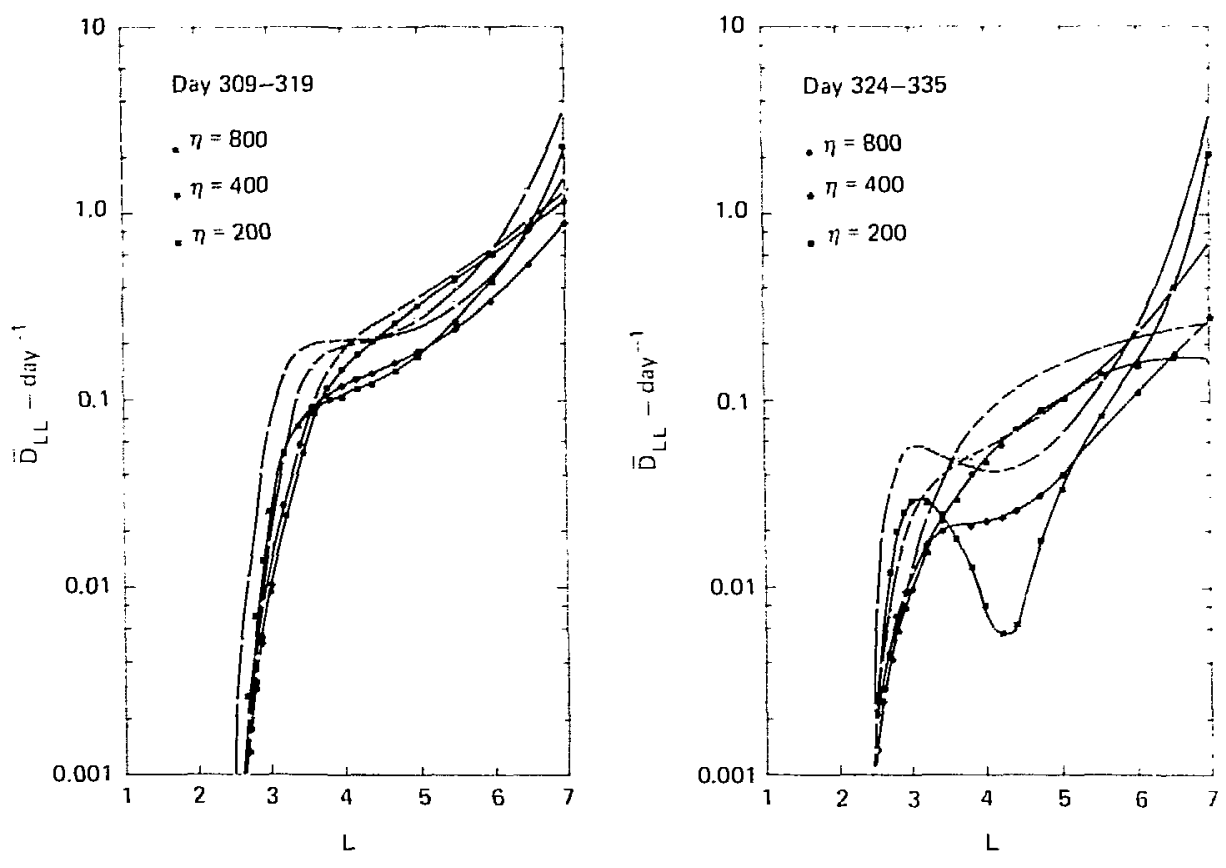

Fig. 27. Calculated diffusion coefficients. The open points represent a conceivable upper limit to the analysis assuming $\partial \mathrm{f}_{0} / \partial t=0$. Results past $L=6$ are an extrapolation only.

Obviously there are errors in the evaluation of $\vec{D}_{L L}$ in this region, but the dip is real. Interestingly, such a dip has been found at about the same $L$ shell in the diffusion coefficient for energetic protons [Söraas, 1969] obtained from Explorer-26 data. We do not know whether this similarity has physical significance. Earlier we presented the decay of 1530-keV electrons for the November period in Fig. 16. We obtained 6.2 d for the decay period for the overall decay but somewhat longer periods for the specific times of the data analysis. For the pitch-angle diffusion lifetime we arrived at $4.2 \mathrm{~d}$. Obviously there is the possibility that the calculated $\bar{\sigma}_{\mathrm{LL}}$ at $L=3$ for both time periods is a bit high, and part of the shoulder on the data plot may have resulted from inaccuracies in the data or in our interpretation. Nevertheless the data analysis indicates weak inward diffusion during the decay of the electrons in Fig. 16. Finally, we focus attention on the $\eta=800$ results. Here the apparent decay rates were always slow compared to the assigned pitch-angle diffusion rates, and there would seem to be no 

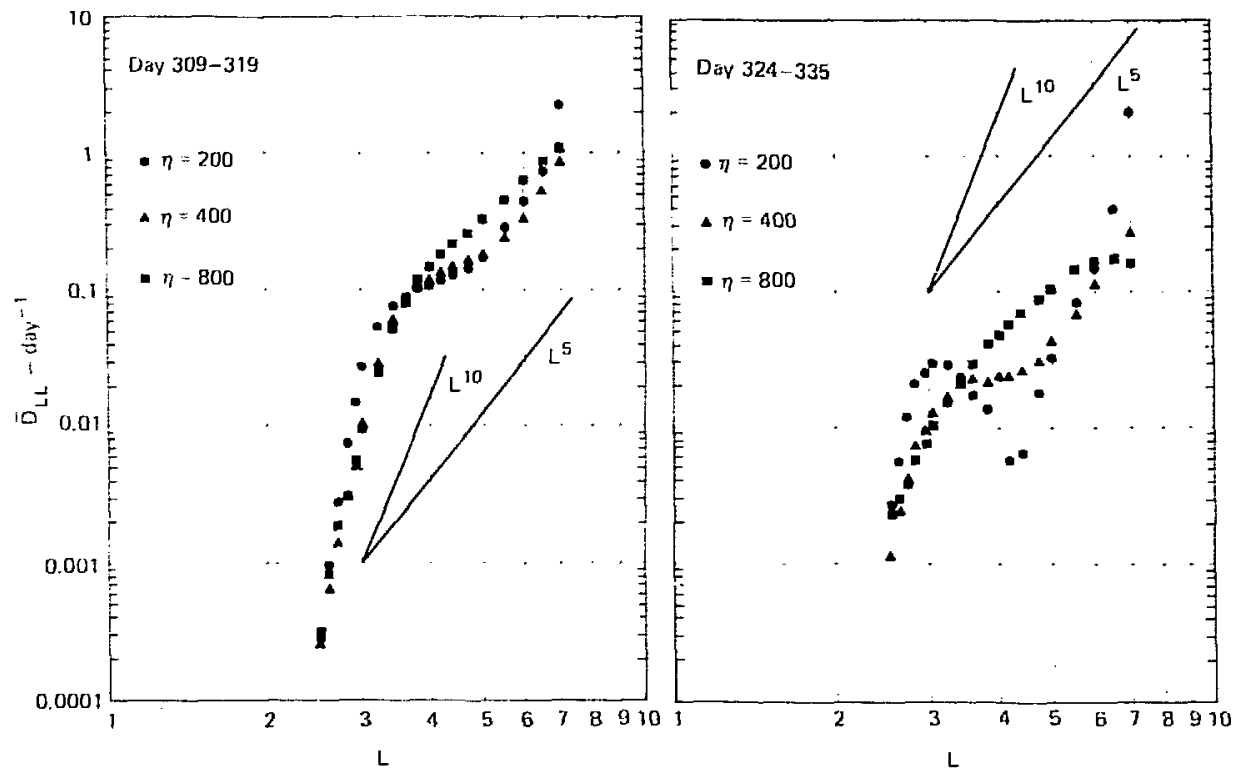

Fig. 28. Final values of $D_{L L}$. Power law variations are included to facilitate comparison to theortical results. Results past $L=6$ are an extrapolation on Ty.

possibility of error from that source; the use of the integral method minimizes any possible errors in the value of $\bar{D}_{L L}$ due to inaccuracies in $\partial f_{0} / \partial L$.

A possible general criticism exists in the radial-diffusion analysis of our November 1968 data. The data were acquired at moderate latitudes, including the pitch-angle diffusion decay rates, but the $j_{1}$-fluxes were transformed to the equator for reasons that are no longer pertinent. Preferably, the data should be re-analyzed at $45.7^{\circ}$, an equatorial pitch angle that minimizes drift-shell and model-dependent effects. The pitch-angle corrections would be more accurate, and, using the appropriate distribution function and diffusion equation ( $n=2.75)$, the analysis would be more consistent. However, we have confined our diffusion analysis to the higher $\eta$ values and, because of this, do not believe that the new results would differ greatly from those of the present analysis.

The present results are compared with those of some earlier investigations in Fig. 29. Our results (curve 1) are shown with verticill bars (that are not error bars) showing the range in variation of our results. 


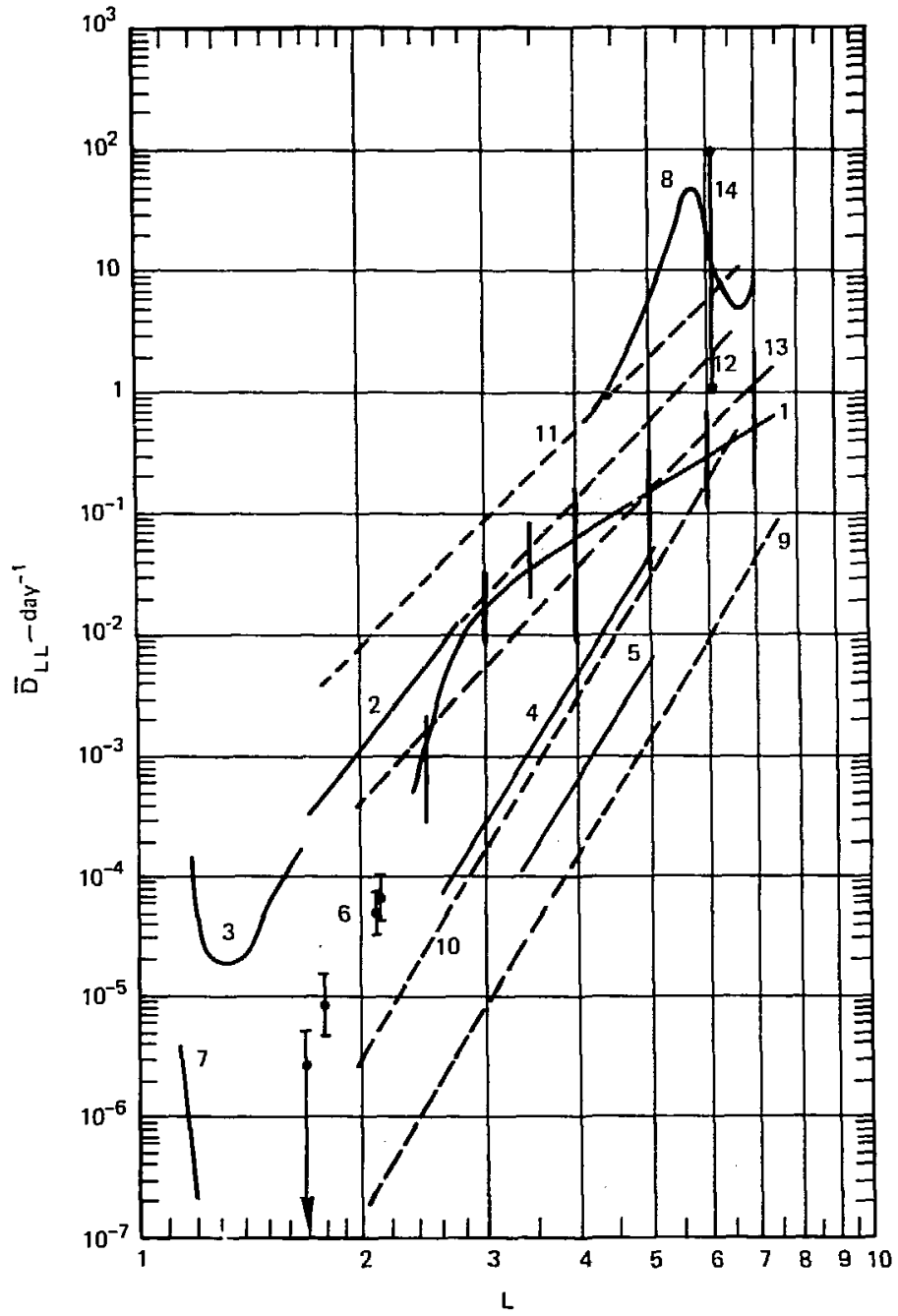

Fig. 29. Present results compared to the other results, both experimental and theoretical. The bars on Curve 1 represent the range of our results. Curves (2)-(8) are experimental and come from Tomassian et al. [1972] (2), Farley [1969] (3), Newkirk and Walt [1968b] (4), Lanzerotti [1970] (5), Wal 1970] (6), Newkirk and Wa7t [1968a] (7), and Kavanaugh [1968] (8). Curves -114) are theoretical or semitheoretica] and come from Nakada and Mead [1905] (9), Tverskay [1965] (10), Birmingham [1969] (11), Cornwa11 [1968]and Iomassian [1972] (12 and 13), and Holzworth and Mozer [1979] (14). 
Curve? [Tomassian et al., 1972] shows results following the storm on September 2, 1966. Significantly greater injection occurred at low shells for this day than for October 31-November 1, 1968, despite the som- nat-1ower Dst. In comparing these data to ours, it is well to note that they analyzed electrons with $\eta=16.2-33.4$, whereas the lowest $n$ for which we have done the analysis is 200. Curve 3 [Farley, 1969 ] comes from the decay of Starfish electrons. (These data are incorrectly quoted in most subsequent publications [Farley, Walt, private communication, 1979]. When examining older data, the reader must check whether $\mathrm{D}_{2}$, the mean-square deviation, was reported rather than $\left.\bar{D}_{\mathrm{LL}} ; \overline{\mathrm{D}}_{\mathrm{LL}}=\mathrm{D}_{2} / 2.\right)$ Curves 4 and 5 [Newkirk and WaIt, 1968b and Lanzerotti et al., 1970], respectively, describe the decay of outer-belt electrons. Curve 6 [Walt, 1970; Walt, private communication, 1979] comes from measurements of artificially injected electrons, and Curve 7 [Newkirk and Walt, 1968a] is an analysis of effects on the inner edge of the inner belt and is considered the lower limit for the results [Walt and Farley, 1979]. Curve 8 [Kavanaugh, 1968] is an early result in the outer belt regions; the dashed curves that follow in our discussion are theoretical or semi-theoretical. Curve 9 [Nakada and Mead, 1965] is an estimate of the diffusion that can be obtained from the observed level of magnetic impuises. Curve 10 [Tverskoy, 1965] is the result of a somewhat similar calculation. Curit: "Birmingham, 1969] shows the results of using a $0.2 \mathrm{mV} / \mathrm{m}$ electric field and a $i-\mathrm{hr}$ correlation time between electric field and particle drifts. Curves 12 and 13 were derived for $\eta=50$ and 800 , respectively, using a formula derived by Cornwall [1968] but with the adjustable parameters provided by Tomassian et al., [1972]. The convection electric field was set at $0.28 \mathrm{mV} / \mathrm{m}$ and the characteristic time at $1600 \mathrm{~s}$. Curve 14 shows recent measurements of Holzworth and Mozer [1979]. Ionospheric electric-field measurements were used, the E-fields mapped along the B-field lines, and $\mathrm{D}_{\mathrm{LL}}$ calculated. The upper range is for $25 \mathrm{keV}$, and the lower range is for $1.2 \mathrm{MeV}$. Although the results seem a bit high, they indicate the dominance of electric fields in the diffusion.

The results of the Cornwall [1968] equation with the parameters provided by Tomassian et a1. [1972] are in reasonable agreement with the present results for $L \geq 3$. Clearly the present results show that the diffusion coefficient is not constant but varies depending upon conditions. We feel that the results of the diffusion analysis for Days 309-319 and 324-335 are of sufficient accuracy that their difference must be taken seriously. It would 
appear that the Tomassian results ( $L=1.7$ to 2.7$)$ were strongly dominated by electric diffusion and similarly for our results for $L \geq 2.8$. However, there is an inescapable turn down in our results at $L \leq 2.8$ that seems to approach the results of curve 6 (which cones from temporal changes in features of bomb-injected electrons) and the extension of curve 4 . It seems reasonable to suggest that a magnetic interaction was doninant for these cases.

We now compare our pitch-angle diffusion data with data in the literature. Figures 30a-d show Figs. 39-41 from Schulz and Lanzerotti [1974] and Fig. 4 from Tomassian et al. [1972]. Figure 30a shows Explorer-26 data [Wi1liams Et al., 1965], Fig. 30b shows Explorer-26 and 1963 38c data [Williams et a].. 1965], Fig. 30c shows data acquired by measurements on a number of satellites [Roberts, 1969], anri Fig. 30d shows the data used by Tomassian et al. [1972] in the analysis of the radial diffusion data discussed earlier. Figures 30 ab clearly show rapid decays comparable to our present measurements. Figure $3 \mathrm{~L}$. is especially interesting. In the first time interval, we interpret the measurenents as showing predominantly pitch-angle diffusion whereas the latter interval is dominated by radial diffusion. At first glance, the data of Fig. 30c seem in disagreement with our results, but that is not really the case if one makes allowances for the effective electron spectrum that these experiments prabably obseried. It might be interesting to make a detailed comparison of each of these results with our present results, weighting the spectrum with respect to decay rates, but such is beyond the scope of the present work. The data in Fig. $30 d$ used by Tomassian et al. are something of an enigma. We expect that the authors were overly influenced by previous results and did not make full use of their ov3-3 data. Clearly, this means that the values of $\bar{D}_{L L}$ for the upper $L$ range $(\geq 2.5)$ in their results are $10 w$ by as much as a factor of two. This is a small error considering the large variations among previously reported values.

The best theoretical results for outer belt electrons are those of Lyons, Thorne, and Kennel [1972], which are based on the interactions of the electrons with whistler-mode radiation. A comparison with our results is provided in Fig. 22 (from Fig. 7 of Lyons, Thorne, and Kennel). The qualitative behavior of these results is much like ours. The agreement at $L=2.5$ and 3.0 seems particularly good, suggesting that with a more judiciously chosen wave-spectrum, the theoretical result would agree perfectly. The argreement at $L=4.0$ is not nearly as good, and that at 5.0 is even worse. it would appear that the effects of whistier-mode interactions 

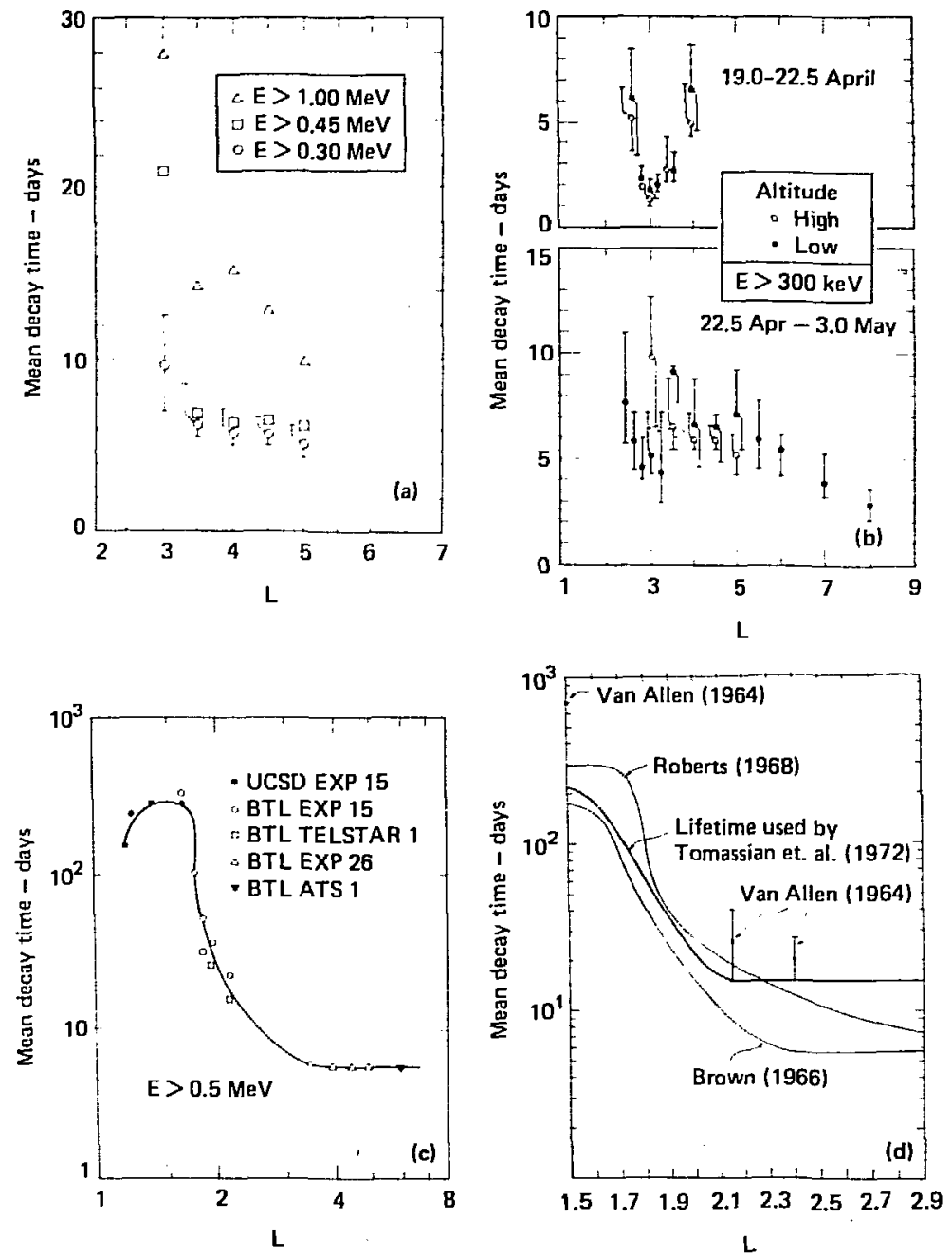

Fig. 30. Other measurements of energetic-electron lifetimes. Panels a, b, and $c$ are from Schulz and Lanzerotti $[1974]$, and Panel $d$ is from Tomassian et al. [1972].

are dominant in the slot region but become less so at the high $L$ shells, which are probably outside of the plasma sphere most of the time.

By searching for the lowest lifetimes in the decays of the two data sets, we feel we have identified the pitch-angle diffusion lifetimes. That it is possible to make such an identification will certainly be challenged, but we 
feel that the total analysis argues for our assumption that the most rapid decays are those observed when radial diffusion is negligible. As mentioned earlier, the fact that the pitch-angle diffusion lifetimes are more or less constant argues that the wave spectrum required to drive the diffusion is nearly constant. Russell (private comunication, 1979) tells us that the UCLA-JPL search-coil experiment on Ogo 5 showed about the same wave spectrum from pass to pass in the regions of the outer belt inside the plasma pause. Russe 11 has not cione a careful quantitative comparison of the data, however; such would be a valuable contribution to understanding pitch-angle diffusion on the lower L shells.

\section{ACKNOWLEDGMENTS}

Several people were involved in deyolnoing the codes at LLL used specifically for this work: Earbarba W. Gumm, Robert W. Kuhn, and Keith W. Johnson. We thank A. Vampola fi, the use of his ov3-3 data. Special thanks go to $\mathrm{C}$. T. Russell for the use of his magnetometer data acquired on 0go 5 ; without these data, this report would not have been possible. The work at LLL was performed under Department of Eriergy contract No. W-7405-Eng. 48 and U.S. Air Force Office of Scientific Research Support Agreement No. AFOSR-ISSA-77-12. The work at LPARL was performed under the Lockheed Independent Research Funding and under LLL Work Agreement No. 2182309.

\section{REFERENCES}

Aubry, M. P., M. G. Kivelson, R. L. McPherron, C. T. Russell, and D. S. Colburn, A study of the outer magnetosphere near midnight at quiet and disturbed times, J. Geophys. Res., 77, 5487, 1972.

Birmingham, T. J., Convection electric fields and the diffusion of trapped magnetospheric radiation, J. Geophys. Res., 74, 2169, 1969.

Bostrom, C. 0., D. S. Beall, and 3. C. Armstrong, Time history of the inner radiation zone, October 1963 to Oecember 1968, 3. Geophys Res., 75, 1246, 1970. 
Buck, R. M., and H. I. West, Jr., Energetic electrons in the inner belt in 1968: Fits to Lawrence Livermore laboratory's magnetic electron spectrometer data obtained on 0go-5, Lawrence Livermore Laboratory, Livermore, Calif., UCRL-51710, 1974.

Chan, K. H., M.J. Teague, N. J. Schofield, and J. I. Vette, Modeling of electron time variations in the radiation belts, in Quantitative Modeling of Magnetospheric Processes (Geophysical Monograph 21), W. P. 01son, Ed., Amer. Geophys. Union, Washington, D. C., 1979, p. 121.

Cladis, J. B., G. T. Davidson, and L. L. Newkirk, The Trapped Radiation Handbook, DASIC, General Electric Company-TEMP, Santa Barbara, Calif., 1974.

Cornwall, J. M., Diffusion processes influenced by conjugate point wave phenomena, Radio Sci., 3 , 740, 1968.

Davidson, G. T., Relativistic electron precipitation and resonance with ion cyclotron waves, J. Atmos. Terr. Phys., 40, 1085, 1978.

Far-ley, T. A., Radial diffusion of Starfish electrons, J. Geophys. Res., 74, $3591,1969$.

Hilberg, R. H., M. J. Teague, and J. I. Vette, Comparison of the trapped electron models $A E-4$ and $A E-5$ with $A E-2$ and $A E-3$, National Space Science Data Center, Greenbelt, Md., NSSOC 74-13, 1974.

Holzworth, R. H., and F. S. Mozer, Direct evaluation of the radial diffusion coefficient near $L=6$ due to electric field fluctuations, J. Geophys. Res., 84, $2559,1979$.

Kavanaugh, L. D., Jr., An empirical evaluation of radial diffusion coefficients for electrons of 50-100 keV from $L=4$ to $L=7$, J. Geophys. Res., 73, 2959, 1968.

Kennel, C. F., and H. E. Petschek, Limit on stably trapped particle fluxes, J. Geophys. Res., 71, 1, 1966. 
'anzerctti, L. J., C. G. Marlennan, and M. Schulz, Radial diffusion of outerzone electrons: An empirical approach to third-invariant violation, $\underline{J}$. Geophys. Res., 75, 5351, 1970.

Lyons, L. R., R. :. Thorne, and C. F. Kennel, Electron pitch-angle diffusion driven by oblique whistler-mode turbulence, 3. Plasma Phys., 6, 589, 1971.

Lyons, L. R., R. M. Thorne, and C. F. Kennel, Pitch-angle diffusion of rariation belt electrons within the plasmasphere. J. Geophys Res., 77, 3455, 1972.

Lyons, L. R., and R. M. Thorne, Parasitic pitch angle diffusion of radiation belt particles by ion cyclotron waves, J. Geophys. Res., 77, 5608, 1972.

Lyons, L. R., and R. M. Thorne, Equilibrium structure of radiation belt electrons, J. Geophys. Res., 78, 2142, 1973.

Lyons, L. R., and D. J. Williams, The quiet-time structure of energet ic (35-560 keV) radiation belt Electrons, J. Geophys Res., 80, 943, 1975.

Hakada, M. P., and G. D. Mead, Diffusion of protons in the outer radiation belt, J. Geouhys. Res., 70, $4777,1965$.

Newkirk, L. L., and M. Walt, Radial diffusion coefficients for electrons at low L-values, J. Geophys. Res., 73, 1013, 1968a.

Newkirk, L. L., and M. Walt, Radial diffusion coefficients for electrons at $1.76<\mathrm{L}:<5$, J. Geophys. Res., 73, 7231, 1968b.

01 son, W. P., and K. A. Pfitzer, A quantitative model of the magnetospheric magnetic field, U. Geophys. Res., 79, 3739, 1974.

Pfitzer, K. A., S. Kane, and J. R. Winckler, The spectra and intensity of electrons in the radiation belts, Space Res., 6 , 702, 1966. 
Pfitzer, K. A., and J. R. Winckler, Experimental observation of a large addition to the electron inner radiation belt after a solar flare event, $J$. Geophys. Res., 73, 5792, 1968.

Roberts, C. S. Pitch-angle diffusion of electrons in the magnetosphere, Rev. Geophys., 7, 305, 1969.

Schulz, M., Drift-shell splitting at arbitrary pitch angle, J. Geophys Res., 77, $624,1972$.

Schulz, M., and L. J. Lanzerotti, Physics and Chemistry in Space 7: Particle diffusion in the radiation belts, Springer-Verlag, New York, 1974.

Singley, G. W., and J. I. Vette, A model environment for outer zune electrons, National Space Science Data Center, Greenbelt, Md., NSSDC 72-13, 1972.

Söraas, F., Comparison of post-storm non-adiabatic recovery of trapped protons with radial diffusion, Goddard Space Flight Center, Greenbelt, Mi., GSFC $x-612-69-241,1969$.

Sugiura, H., and Poras, D. J., Hourly values of equatorial Dst for the years 1957 to 1970, Goddard Space Flight Center, Greenbelt, Md., GSFC $\times-645-71-278,1971$.

Teague, M. J., K. W. Chan, and J. I. Vette, AE6: A model environment of trapped electrons for solar maximum, National Space Science Data Center, Greenbelt, Md., NSSDC/WDG-A-R\& S 76-04, 1976.

Teague, M. J., and J. I. Vette, A model of the trapped electron population for solar minimum, National Space Science Data Center, Greenbelt, Md., NSSDC 74-03, 1974.

Thorne, R. M., and C. F. Ken:: Relativistic electron precipitation during magnetic storm main phase, J. Geophys Res., 76, 4446, 1971.

Tomassian, A. D., T. A. Farley, and A. L. Vampola, Innel-Zone energetic electron repopulation by radial diffusion, J. Geophys. Res., 77, 3441, 1972. 
Tverskoy, B. A., Transport and acceleration of charged particles in the ea.th's magnetosphere, Geomagn. Aeron., USSR, 5, 617, 1965.

Vampola, A. L., Eiectron pitch angle scattering in the outer zone during magnet ically disturbet time, J, Geophys Res., 76, 4685, 1971.

Vampola, A. L., Natural variation in the geomagnetically trapped electron population, Proceedings of the National Symposium on Natural and Man-Made Radiation in Space, NASA Tech. Memo T: X02440-359-547, 1972, p. 539.

Vampola, A. L., J. B. Blake and G: A. Paulikas, A new study of the outer zone magnetospheric electron environment, J. Space Craft and Rockets, 14, 690, 1977.

Vette, J. I., Magnetospheric particle populations, in Earth's Magnetospheric Processes, B. M. McCormac, Ed,, D. Reidel Publishing Co., Dordrecht, Holland, 1972, p. 53.

Walt, M., Radial diffusion of crapped particles, in Particles and Fields in the Magnetosphere, B. M. McCormac, Ed., D. Reidel Pubiishing Co., Dordrecht, Holl and, 1970 , p. 410.

West, H. I., Jr., J. H. Wujeck, J. H. McQuaid, J. C...Jenson, R. G. D'Arcy, $\mathrm{Jr}$., R. W. Hill, and R. M. Bogandowicz, The LRL electron and proton spectrometer on NASA's Orbiting Geophysical Observatory $V(E)$, Lawrence Livermore Laboratory, Livermore, Cal if..,... UCRL-50572, 1969.

West, H. I., Jr., and R. M. Buck, Pitch angle distributions of energetic electrons in the equatorial regions of the outer magnetosphere-0go 5 observations, in Magnetuspheric Physic, B. M. McCormac, Ed., D. Reide 1 Publishing Co., Dordrecht, Holland, 1970, p. 93.

West, H. I., Jr., R. M. Buck, and J. R. Walton, Electron spectra in the slot and the outer radiation belt, EOS Trans. Am. Geophys. Union, 51, 806, 1970. 
Weat. H. I., ir., W. M. Buck, and 1. R. Walton, The LLL Eleretron and protor. ipartraneter on NA.SA, orbiting Geophysical observatory 5 (Final report for Exper iment 6). Lamence Livermore Laboratory, Livermore, Ca., UCRL-51325, 1973 \%

Wert, H. I., ir., P. M. Bur.k, ard J. P. Walton, Electron piteh angle tistibution: throughout the magnetosphere as observed on ogo 5, J. Geopnys. Fer., 78, 1064, $1973 \mathrm{~b}$.

Wes, II. I., Jr., P. M. Buck, and J. R. Walton, ratellite studiar of magretospheric substorms on August 15, 1968: 6. Ogo 5energetir fiectron vbservetions--pitch angle distributions in the nighttime magnetosphere, $]$. Grophys. Plos., 78, 3093, 1973r.

West, H. I., Jr., and R. M. Buck, Energetic electrons in the inner belt in 1963, Planet. Space Sci., 42, 643, 1976 a.

West, H. J., Jr., and R. M. Buck, A study of electron spectra in the inner beit, J. Geophys. Res., 81, 4696, 1976 b.

West, H. I., Jr., The signatures of the vario.s regions of the outer magnetosphere in the pitch angle distributions of energetic particles, in Duantitative Modeling of Magnetospheric Processes (Geophysical Monograph 21), W. P. 01son, Ed., Amer. Geophys. Union, Washington, D. C., 1979, p. 150.

Williams, D. J., J. F. Arens, and L. J. Lanzerotti. Observations of trapped electrons at low and high altitudes, J. Geophys. Res., 73, 5673, 1968.

Winckler, J. R., The origin and distribution of energetic electrons in the Van Allen radiation belts, in Particles and Fields in the Magnetosphere, $B . M$. McCo;mac, Ed., D. Reidel Publishing Co., Dordrecht, Holland, 1970, p. 332.

Zeligman, M. M., and J. R. Walton, The LLL electron and proton spectrometer on NASA's Orbiting Geophysical Observatory $V(E)$ : The three-way merged tape (an archival data base), Lawrence Livermore Laboratory, Livermore, Calif., UCRL-51314, 1972. 